NIST Technical Note 2082

Second Edition

\title{
The Structure of Medium-Scale Pool \\ Fires
}

\author{
Ryan Falkenstein-Smith \\ Kunhyuk Sung \\ Jian Chen \\ Kimberly Harris \\ Anthony Hamins
}

This publication is available free of charge from:

https://doi.org/10.6028/NIST.TN.2082e2

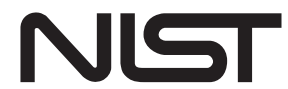

National Institute of Standards and Technology

U.S. Department of Commerce 


\title{
NIST Technical Note 2082 Second Edition
}

\section{The Structure of Medium-Scale Pool Fires}

\author{
Ryan Falkenstein-Smith \\ Kunhyuk Sung \\ Jian Chen \\ Anthony Hamins \\ Fire Research Division \\ Engineering Laboratory \\ Kimberly Harris \\ Chemical Science Division Material \\ Measurement Laboratory
}

This publication is available free of charge from:

https://doi.org/10.6028/NIST.TN.2082e2

January 2022

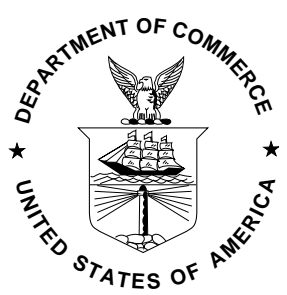

U.S. Department of Commerce Gina M. Raimondo, Secretary

National Institute of Standards and Technology James K. Olthoff, Performing the Non-Exclusive Functions and Duties of the Under Secretary of Commerce for Standards and Technology \& Director, National Institute of Standards and Technology 
Certain commercial entities, equipment, or materials may be identified in this document in order to describe an experimental procedure or concept adequately. Such identification is not intended to imply recommendation or endorsement by the National Institute of Standards and Technology, nor is it intended to imply that the entities, materials, or equipment are necessarily the best available for the purpose.

National Institute of Standards and Technology Technical Note 2082, Second Edition

Natl. Inst. Stand. Technol. Tech. Note 2082, Second Ed., 85 pages (January 2022)

CODEN: NTNOEF

This publication is available free of charge from: https://doi.org/10.6028/NIST.TN.2082e2 


\begin{abstract}
This report documents a series of time-averaged gas species measurements made along the centerline of methanol, ethanol, acetone, methane, and propane pool fires steadily burning in a quiescent environment. All gas species measurements are obtained using a Gas Chromatograph/ Mass Spectrometer System (GC/MS) for gas samples extracted at various heights above the fire and repeated at least twice at each location. Gas species volume fractions are determined via the GC/MS using predetermined calibration factors. Soot mass fractions are simultaneously measured during the gas sampling process. The gas species volume and soot mass fractions are compared at different heights within the fire and across a variety of different fuels. Other fire parameters are measured as well, including timeaveraged temperature and velocity measurements and mass burning rates.
\end{abstract}

\title{
Keywords
}

Acetone, Ethanol, Gas pool fires, Gas species measurements, Liquid pool fires, Methane, Methanol, Propane 


\section{Table of Contents}

1 Introduction $\quad 10$

2 Description of Experiments $\quad 11$

2.1 Liquid Pool Burner Setup 11

2.2 Gas Pool Burner Setup 12

2.3 Measuring Flame Characteristics $\quad 12$

2.4 Centerline Temperature Measurements $\quad 12$

2.5 Centerline Velocity Measurements 15

2.6 Measuring the Volume Fraction of Gas Species via GC/MS 16

2.7 Determining Soot Mass Fraction 21

3 Results $\quad 23$

3.1 Flame Observations 23

3.2 Comparison of Pool Fires from Different Fuels 23

4 Verifying Gas Species Measurements $\quad 28$

4.1 Counting Moles 28

4.2 Carbon to Hydrogen Ratio 29

4.3 Plotting the Results in Mixture Fraction Space 31

5 Conclusion $\quad 38$

References $\quad 39$

A Uncertainty Analysis of Pool Fire Parameters 41

A.1 Mass Burning Rate $\quad 41$

A.2 Heat Release Rate and $Q^{*} \quad 41$

A.3 Mean Flame Height 41

A.4 Uncertainty Analysis of Temperature Measurements 41

A.5 Uncertainty Analysis of Velocity Measurements 42

B Figures of Time-Averaged Bead Temperature Readings 43

B.1 Methanol 43

B.2 Ethanol 44

B.3 Acetone 45

B.4 Methane 46

B.5 Propane $(21 \mathrm{~kW}) \quad 47$

B.6 Propane $(34 \mathrm{~kW}) \quad 48$

C Estimating the Characteristic Length of the Regime of Influence 49

D Elution times of combustion species using a Select for Permanent Gasses$\begin{array}{ll}\text { Dual Column } & 51\end{array}$

E Uncertainty Analysis of Gas Species Concentrations

E.1 Uncertainty of Volume Fractions 52

E.1.1 Number of Moles of a Given Species $\quad 52$ 
E.1.2 Total Number of Moles Identified 52

E.2 Uncertainty of Mass Fractions $\quad 52$

E.2.1 Uncertainty of the average molecular weight 53

F Uncertainty Analysis of Gas Species Calibrations $\quad \mathbf{5 4}$

F.1 Total Moles Injected into the GC/MS for Calibation 55

F.2 Table of Gas Standards with Error $\quad 56$

F.3 Concentration of Vapors from Bubblers $\quad 57$

G Uncertainty Analysis of the Soot Mass Fraction 59

G.1 Mass of Soot 59

G.2 Mass Flow Controller Volumetric Flow Rate 59

G.3 Total Mass Detected $\quad 60$

H Figures of Averaged Volume Fractions $\quad 61$

$\begin{array}{lll}\text { H.1 Methanol } & 61\end{array}$

$\begin{array}{lll}\text { H.2 Ethanol } & 62\end{array}$

H.3 Acetone $\quad 64$

H.4 Methane 66

$\begin{array}{lll}\text { H.5 Propane }(21 \mathrm{~kW}) & 68\end{array}$

$\begin{array}{ll}\text { H.6 Propane }(34 \mathrm{~kW}) & 70\end{array}$

I Figures of Averaged Mass Fractions $\quad 72$

$\begin{array}{ll}\text { I.1 Methanol } & 72\end{array}$

$\begin{array}{lll}\text { I.2 Ethanol } & 73\end{array}$

$\begin{array}{lll}\text { I.3 Acetone } & 74\end{array}$

$\begin{array}{lll}\text { I.4 Methane } & 75\end{array}$

$\begin{array}{lll}\text { I.5 Propane }(21 \mathrm{~kW}) & 76\end{array}$

$\begin{array}{ll}\text { I.6 Propane }(34 \mathrm{~kW}) & 77\end{array}$

J Uncertainty Analysis of the Verification Scheme $\quad 78$

$\begin{array}{lll}\text { J.1 Ratio of moles identified to moles injected } & 78\end{array}$

$\begin{array}{lll}\text { J.2 Carbon to Hydrogen Ratio } & 78\end{array}$

$\begin{array}{lll}\text { J.3 Inert Ratio } & 78\end{array}$

J.4 Uncertainty Analysis of the Mixture Fraction $\quad 78$

K Previous Measurements in $30 \mathrm{~cm}$ Pool Fires $\quad 80$ 


\section{List of Tables}

Table 1 List of measurements and thermochemical properties of fuels 25

Table F.1 Gas standards used to calibrate the GC/MS 56

Table F.2 Liquid vapor pressure correlation coefficients for various calibrated liquids 58

Table K.1 List of previously measure pool fire parameters $\quad 80$

Table K.2 Previously reported pool fire measurements 82 


\section{List of Figures}

Fig. $1 \quad$ Photograph of the burner 11

Fig. 2 Photographs of the fuel monitoring process 13

$\begin{array}{lll}\text { Fig. } 3 & \text { Photograph of the gaseous burner } & 14\end{array}$

Fig. 4 Photograph of a bi-directional probe 15

$\begin{array}{lll}\text { Fig. } 5 \text { A schematic of the gas sampling procedure } & 17\end{array}$

Fig. 6 A schematic of the internal plumbing system in the gas chromatograph 19

Fig. 7 Chromatogram of a pool fire gas sample 20

Fig. 8 Process for cleaning soot probe 22

Fig. 9 Photographs of pool fires during their pulsing cycles 24

Fig. 10 Mean gas temperature profiles 26

Fig. 11 Mean centerline velocity profiles 26

Fig. 12 Centerline volume fraction and soot mass fraction profiles 27

Fig. 13 Ratio of moles identified to moles injected 28

Fig. 14 Carbon to hydrogen ratio calculated from all species 29

Fig. $15 \mathrm{Ar} / \mathrm{N}_{2}$ ratio within the fire envelop compared to ambient air 30

Fig. 16 Mean mass fractions as a function of mixture fraction, methanol 32

Fig. 17 Mean mass fractions as a function of mixture fraction, ethanol 33

Fig. 18 Mean mass fractions as a function of mixture fraction, acetone 34

Fig. 19 Mean mass fractions as a function of mixture fraction, methane 35

Fig. 20 Mean mass fractions as a function of mixture fraction, propane $(21 \mathrm{~kW}) \quad 36$

Fig. 21 Mean mass fractions as a function of mixture fraction, propane (34 kW) 37

Fig. B.1 Mean and RMS centerline bead temperature profile of nethanol 43

Fig. B.2 Mean and RMS centerline bead temperature profile of ethanol 44

Fig. B.3 Mean and RMS centerline bead temperature profile of acetonel 45

Fig. B.4 Mean and RMS centerline bead temperature profile of methane 46

Fig. B.5 Mean and RMS centerline bead temperature profile of a $21 \mathrm{~kW}$ propane fire 47

Fig. B.6 Mean and RMS centerline bead temperature profile of a $34 \mathrm{~kW}$ propane fire 48

Fig. C.1 A 2D vector flow field with a sink placed at the origin 49

Fig. F.1 Flow diagram for bubble calibration system used for liquid materials $\quad 57$

Fig. H.1 Volume fractions of major species in the methanol plume 61

Fig. H.2 Volume fractions of major species in the ethanol plume 62

Fig. H.3 Volume fractions of minor and trace species in the ethanol plume 63

Fig. H.4 Volume fractions of major species in the acetone plume 64

Fig. H.5 Volume fractions of minor and trace species in the acetone plume 65

Fig. H.6 Volume fractions of major species in the methane plume 66

Fig. H.7 Volume fractions of minor and trace species in the methane plume $\quad 67$

Fig. H.8 Volume fractions of major species in the propane $(21 \mathrm{~kW})$ plume 68

Fig. H.9 Volume fractions of minor and trace species in the propane $(21 \mathrm{~kW})$ plume 69

Fig. H.10 Volume fractions of major species in the propane $(34 \mathrm{~kW})$ plume $\quad 70$

Fig. H.11 Volume fractions of minor and trace species in the propane $(34 \mathrm{~kW})$ plume 71 
Fig. I.1 Species mass fractions superimposed on methanol state relations

Fig. I.2 Species mass fractions superimposed on ethanol state relations

Fig. I.3 Species mass fractions superimposed on acetone state relations

Fig. I.4 Species mass fractions superimposed on methane state relations

Fig. I.5 Species mass fractions superimposed on propane $(21 \mathrm{~kW})$ state relations

76

Fig. I.6 Species mass fractions superimposed on propane $(34 \mathrm{~kW})$ state relations

77 


\section{Nomenclature}

\section{A Peak area}

$A_{\text {corr }} \quad$ Corrected peak area

C Total number of carbon atoms

$C_{\text {vap }} \quad$ Volume fraction of vapors

$c_{p_{a i r}} \quad$ Specific heat of ambient air

$c_{\mathrm{b}} \quad$ Specific heat of thermocouple bead

D Diameter of Pool Fire

$D_{\mathrm{b}} \quad$ Diameter of thermocouple bead

$\varepsilon \quad$ Thermocouple emissivity

$\eta \quad$ Reciprocal of the local fuel equivalence ratio

$g \quad$ Acceleration of gravity

$\Delta H_{c} \quad$ Heat of combustion

$\mathrm{H} \quad$ Total number of hydrogen atoms

$h \quad$ Convective heat transfer coefficient

$k_{\mathrm{g}} \quad$ Thermal conductivity of the gas

$k_{p}(t)$ Instantaneous probe constant

$L_{\mathrm{f}} \quad$ Mean flame height

$\dot{m} \quad$ Mass burning rate

$m_{\mathrm{det}} \quad$ Total mass detected in GC/MS

$m_{\mathrm{s}} \quad$ Mass of soot

$m_{\mathrm{t}} \quad$ Total mass of gas injected into GC/MS

$m_{\text {tot }} \quad$ Total mass of gas sampled $\mu_{\text {air }} \quad$ Dynamic viscosity of air

$n_{i} \quad$ Amount of moles of species i

$n_{\text {inj }}$ Amount of moles injected into GC/MS

$n_{\text {tot }} \quad$ Total amount of moles

$\mathrm{Nu} \quad$ Nusselt number

$P \quad$ Pressure

$\Delta P(t)$ Instantaneous pressure difference

$\Delta P_{c}(t)$ Instantaneous corrected pressure difference

$P_{\infty} \quad$ Ambient pressure

$P_{\text {vap }} \quad$ Vapor pressure

$\phi \quad$ Local equivalence ratio

Pr Prandtl number

$\dot{Q} \quad$ Heat release rate

$R \quad$ Universal gas constant

Re Reynolds number

$\rho_{\text {air }} \quad$ Density of air

$\rho_{\mathrm{b}} \quad$ Density of thermocouple bead

$\rho_{\text {gas }} \quad$ Density of gas

$\rho_{\infty_{\text {air }}}$ Density of ambient air

$s_{i} \quad$ Standard deviation of component $\mathrm{i}$

$\sigma \quad$ Stefan-Boltzmann constant

$T \quad$ Temperature

$T_{\mathrm{b}} \quad$ Measured thermocouple bead temperature

$T_{\mathrm{g}} \quad$ Gas temperature 


\begin{tabular}{|c|c|c|c|}
\hline$T_{\infty}$ & Ambient temperature & $\mathrm{x}$ & $\begin{array}{l}\text { Number of carbon atoms in the fuel } \\
\text { molecule }\end{array}$ \\
\hline & time & & \\
\hline$\tau_{p}$ & Pressure transducer response time & $\mathrm{x}_{i}$ & $\begin{array}{l}\text { Numbers of carbon atoms in the } \\
\text { molecule }\end{array}$ \\
\hline$U_{\mathrm{g}}$ & Velocity of gas & $\bar{Y}_{\mathrm{F}}$ & Mass fraction of fuel molecule \\
\hline$u_{i}$ & Unce & $\bar{Y}_{i}$ & Mean mass fraction \\
\hline $\begin{array}{l}\dot{V} \\
V_{\mathrm{g}}(t)\end{array}$ & $\begin{array}{l}\text { Volumetric flow rate } \\
\text { Instantaneous gas velocity }\end{array}$ & $\mathrm{y}_{i}$ & $\begin{array}{l}\text { Numbers of hydrogen atoms in the } \\
\text { molecule }\end{array}$ \\
\hline$V_{\mathrm{s}}$ & $\begin{array}{l}\text { Volume of sampled gas injected into } \\
\text { GC/MS }\end{array}$ & $Y_{\mathrm{S}}$ & $\begin{array}{l}\text { Mass fraction of soot } \\
\text { Mixture fraction }\end{array}$ \\
\hline$W_{\mathrm{F}}$ & Molecular weight of fuel molecule & $z$ & Vertical spatial coordinate \\
\hline $\mathrm{W}_{i}$ & $\begin{array}{l}\text { Molecular weight of species i } \\
\text { Mean volume fraction }\end{array}$ & $z^{*}$ & $\begin{array}{l}\text { Normalized vertical spatial coordi- } \\
\text { nate }\end{array}$ \\
\hline
\end{tabular}




\section{Preface to Second Edition}

The second edition of this technical note contains additional data not presented in the proceeding edition, including data on the structure of two propane pool fires. Specifically, new data is reported on the centerline profiles of temperature, velocity, and chemical species for $21 \mathrm{~kW}$ and $34 \mathrm{~kW}$ propane gaseous pool fires established on a $37 \mathrm{~cm}$ effective diameter water-cooled sintered-metal burner. Description of the velocity measurements are provided. Centerline velocity measurements are also provided for the methanol, ethanol, acetone, and methane pool fires. 


\section{Introduction}

Computational fluid dynamics (CFD) models are an important component of performancebased design in fire protection engineering. A requirement of their acceptance in the design process is that these models be verified and validated, the latter of which involves comparison with experimental measurements. The primary objective of this report is to provide data for use in fire model validation.

A pool fire is a fundamental focus of study in fire science. The fuel surface is isothermal, flat and horizontal, providing a well-defined boundary condition for modeling. Fuel and product species concentrations and temperatures have a significant influence on the heat feedback to the fuel surface, which directly affects the burning rate. A zone of particular interest is the fuel rich-core between the flame and the pool surface, where gas species can absorb energy that would otherwise have been transferred to the fuel surface. Few studies in the literature have reported local chemical species measurements within the flame envelop.

The purpose of this study is to characterize the spatial distribution of the principal chemical species in moderate-scale pool fires steadily burning in a well-ventilated, quiescent environment. Here, methanol, ethanol, acetone, methane, and propane are the fuels of interest. Methanol is unique among the fuels because it generates no measurable carbonaceous soot. These particular fuels have been selected because the measurements complement those from previous studies, including the mass burning rate, the temperature and velocity fields, radiative emission, flame height, and pulsation frequency $[1,2]$. 


\section{Description of Experiments}

Descriptions of previous pool fire experiments are found in Refs. [2-6]. All experiments are conducted under a canopy hood surrounded by a cubic enclosure, $2.5 \mathrm{~m}$ on a side, made of a double layer wire-mesh screen $(5 \mathrm{mesh} / \mathrm{cm})$ to reduce the impact of room ventilation. All measurements are made once the mass burning rate reaches a steady-state, achieved approximately $10 \mathrm{~min}$ and $2 \mathrm{~min}$ after ignition for the liquid and gas fuels, respectively.

\subsection{Liquid Pool Burner Setup}

The circular, stainless-steel burn pan, made from cold rolled steel, has an outer diameter of $30 \mathrm{~cm}$, a depth of $15 \mathrm{~cm}$, and a wall thickness of $1.6 \mathrm{~mm}$. The lip of the burner is positioned $30 \mathrm{~cm}$ above the floor. As shown in Fig. 1, the burner is placed within an overflow basin, which extends $3 \mathrm{~cm}$ beyond the burner wall. The bottom of the burner is maintained at a constant temperature by flowing water (approximately $20^{\circ} \mathrm{C} \pm 5{ }^{\circ} \mathrm{C}$ ) through the basin. A fuel level indicator is positioned near the center of the burner to maintain a consistent surface level.

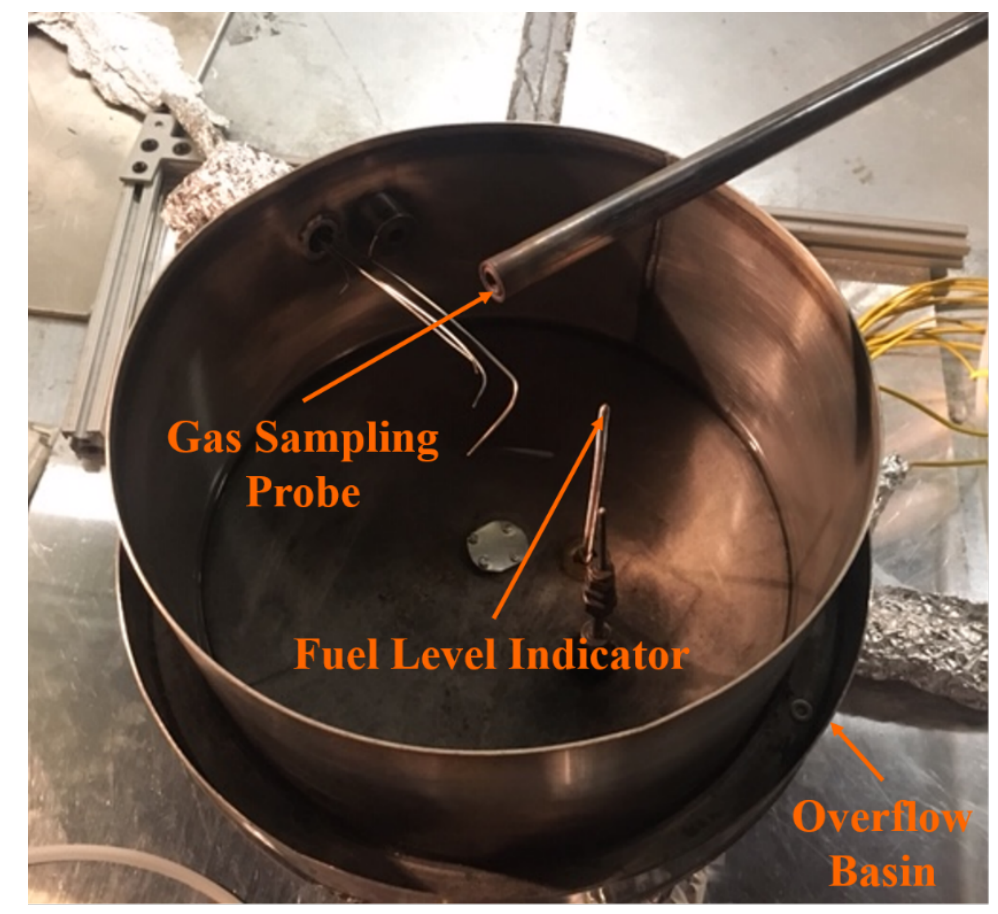

Fig. 1. The $30 \mathrm{~cm}$ burner with fuel level indicator, overflow section, and quenching probe

Fuel to the burner is gravity fed from a reservoir positioned on a mass load cell located outside the enclosure and monitored by a data acquisition system (DAQ). As shown in Fig. 2, the fuel level is monitored via a fuel flow operator. The operator is able to observe a close up of a slightly discernible dimple (approximately $2 \mathrm{~mm}$ ) made from the fuel level 
indicator on the fuel surface using a live video feed. The fuel level is controlled by manually adjusting the fuel flow using a needle valve. The fuel surface is maintained $10 \mathrm{~mm}$ below the burner rim to match previous experimental conditions $[1,2,7,8]$.

\subsection{Gas Pool Burner Setup}

Gas fuels are burned using a burner with an effective diameter of $37 \mathrm{~cm}$ with a flat porous surface of which gaseous fuel is fed through. A photo of the gaseous burner is shown in Fig. 3. Internally, the burner consists of porous sintered metal made of bronze. The pore size is estimates to be approximately $1 / 10 \mathrm{~mm}$. The depth of the porous metal is approximately $8 \mathrm{~cm}$ interlaced with cooling coils. Fuel to the gas burner is controlled via a Brooks mass flow controller, Model $5863^{1}$ located outside of the enclosure. Similar to the liquid pool burner, the gas burner is maintained at a constant temperature by circulating cooled water (approximately $20^{\circ} \mathrm{C} \pm 5{ }^{\circ} \mathrm{C}$ ) through the basin.

\subsection{Measuring Flame Characteristics}

The heat release rate, $\dot{Q}$, is the product of the time-averaged mass burning rate, $\dot{m}$, and the heat of combustion, $\Delta H_{c}$ :

$$
\dot{Q}=\dot{m} \Delta H_{c}
$$

The heat of the combustion is provided by Ref. [9] and reported in Table 1.

The mean flame height, $L_{\mathrm{f}}$, is estimated from 3600 frames of high-resolution video of the experiments using MATLAB's Image Processing Toolbox. Imported color images are decomposed into binary (i.e., black and white) images using a pre-set threshold level. The flame height for a single frame is defined as the distance between the pool surface and flame tip. All measurements are repeated, then averaged to provide the mean flame height. A description of the uncertainty analysis for the mean flame height is described in Appendix A.3.

\subsection{Centerline Temperature Measurements}

Time-averaged temperature measurements are made along the vertical centerline of the fire plumes using S-type (Pt 10\% Rh/Pt), bare-wire, fine diameter thermocouples (OMEGA P10S-001) with wire diameters of approximately $13 \mu \mathrm{m}$ and $25 \mu \mathrm{m}$ for the liquid and gas pool fires, respectively, and bead diameter approximately three times greater. Temperature measurements are sampled at $250 \mathrm{~Hz}$ for $2 \mathrm{~min}$, or approximately 300 pulsing cycles $[10,11]$. The uncertainty of the temperature measurements are discussed in Appendix A.4. In some cases, temperature measurements are made using thermocouples of different configurations. The (uncorrected) mean thermocouple temperature measurements, the cor-

\footnotetext{
${ }^{1}$ Certain commercial products are identified in this report to specify adequately the equipment used. Such identification does not imply a recommendation by the National Institute of Standards and Technology, nor does it imply that this equipment is the best available for the purpose.
} 

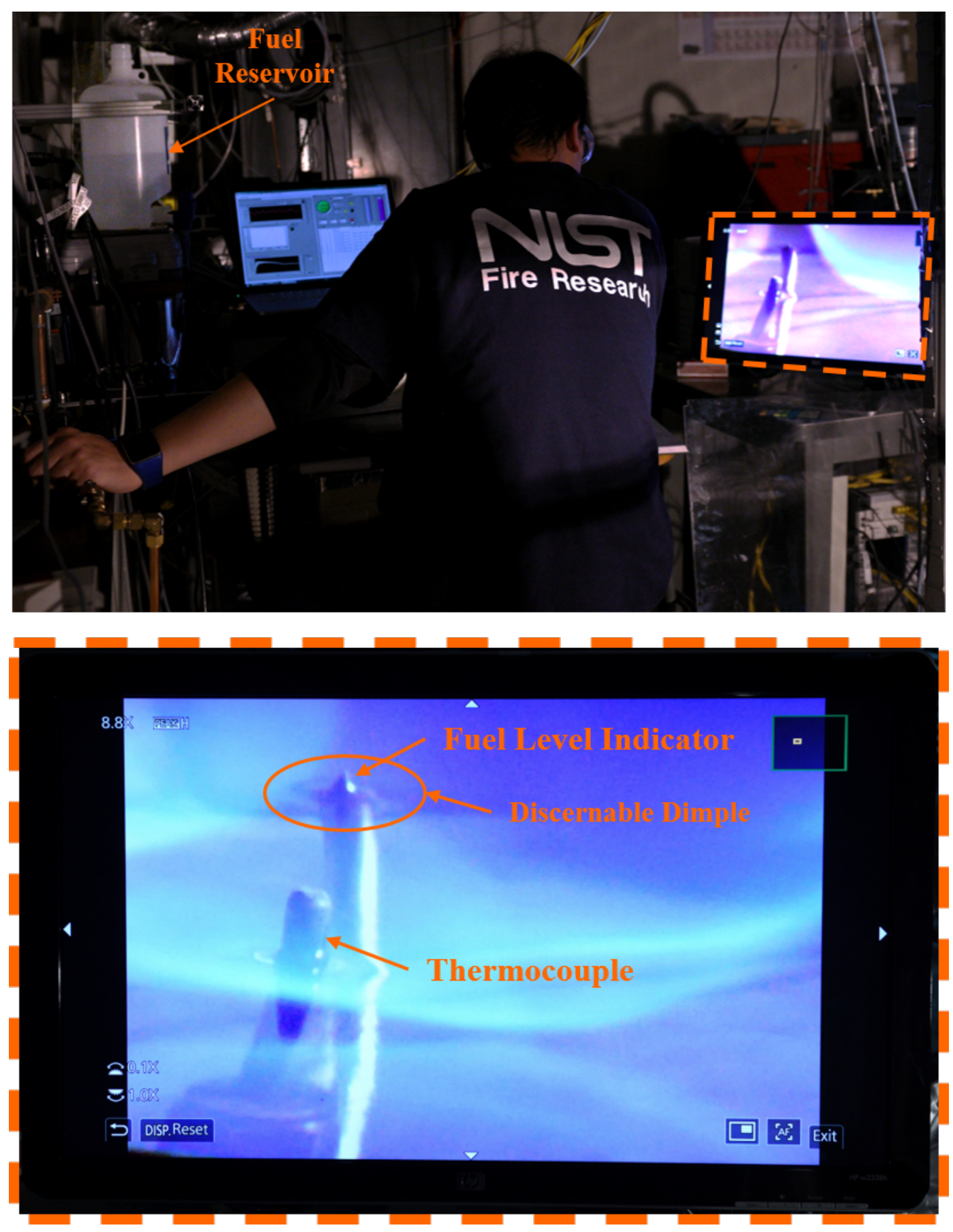

Fig. 2. Photo of fuel flow operator monitoring the fuel level via live video feed (top) and a magnified image of the live video feed used to maintain a consistent fuel level relative to the fuel level indicator (bottom)

rected mean gas temperatures, and the combined uncertainty of the gas measurement for each fire are presented in Appendix B. 


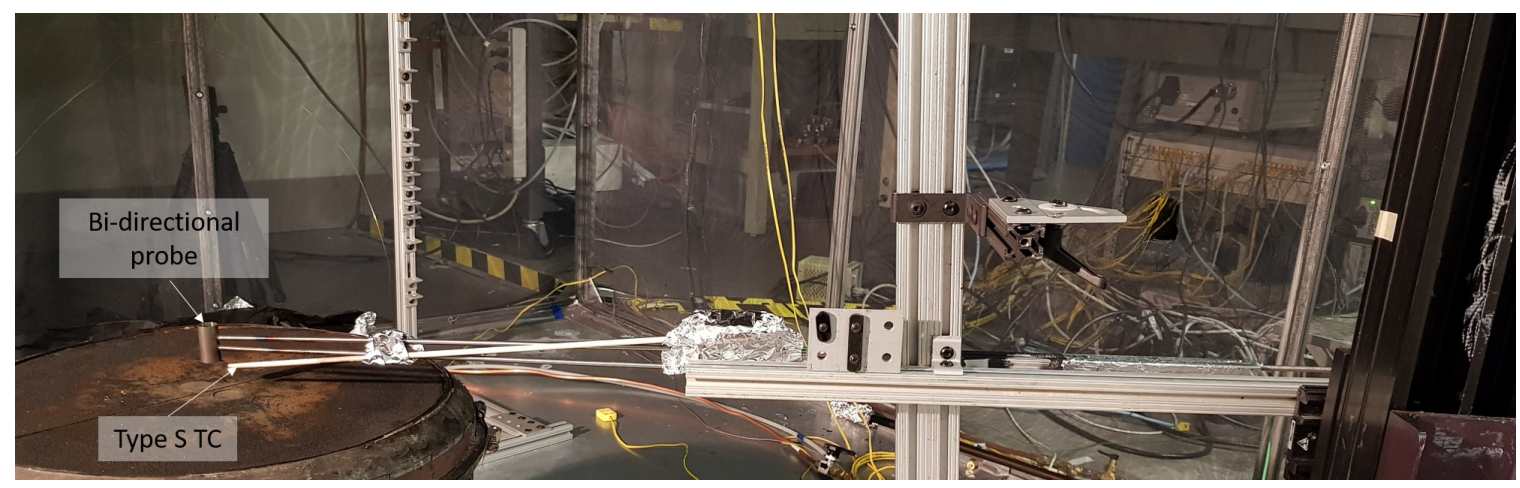

Fig. 3. The $37 \mathrm{~cm}$ effective diameter gaseous burner.

The measured thermocouple temperatures are corrected for heat losses and thermal inertia using the formula described by Shaddix [12]:

$$
T_{\mathrm{g}}(t)=T_{\mathrm{b}}(t)+\tau \frac{\mathrm{d} T_{\mathrm{b}}}{\mathrm{d} t}+\frac{\varepsilon \sigma}{h}\left(T_{\mathrm{b}}(t)^{4}-T_{\infty}^{4}\right)
$$

where $T_{\mathrm{g}}$ is the "true" gas temperature, $T_{\mathrm{b}}$ is the measured bead temperature, $T_{\infty}$ is the ambient temperature, $\sigma=5.67 \times 10^{-11} \mathrm{~kW} /\left(\mathrm{m}^{2} \mathrm{~K}^{4}\right)$ is the Stefan-Boltzmann constant, $\varepsilon$ is the thermocouple emissivity, and $h$ is the convective heat transfer coefficient. The temperature-dependent emissivity of the platinum is taken from Ref. [12] and shown below:

$$
\varepsilon=-0.1+3.24 \times 10^{-4} T-1.25 \times 10^{-7} T^{2}+2.18 \times 10^{-11} T^{3}
$$

$h$ and $\tau$ are defined:

$$
h=\frac{\mathrm{Nu} k_{\mathrm{g}}}{D_{\mathrm{b}}} \quad ; \quad \tau=\frac{\rho_{\mathrm{b}} c_{\mathrm{b}} D_{\mathrm{b}}^{2}}{6 \mathrm{Nu} k_{\mathrm{g}}}
$$

where $k_{\mathrm{g}}$ is the thermal conductivity of the gas, $\rho_{\mathrm{b}}, c_{\mathrm{b}}$, and $D_{\mathrm{b}}$ are the density, specific heat, and diameter of the bead, respectively. The thermal conductivity of the gas is determined from datasets provided in Ref. [13]. The density of the thermocouple bead is assumed constant at $21.45 \mathrm{~g} / \mathrm{cm}$ [14]. The specific heat of the bead is calculated from the formulae provided in Ref. [15]. The Nusselt number, $\mathrm{Nu}$, is calculated using the Ranz-Marshall correlation [12]:

$$
\mathrm{Nu}=2+0.6 \operatorname{Re}^{1 / 2} \operatorname{Pr}^{1 / 3} \quad ; \quad \operatorname{Re}=\frac{\rho_{\text {air }} U_{\mathrm{g}} D_{\mathrm{b}}}{\mu_{\text {air }}} \quad ; \quad \operatorname{Pr}=0.7
$$

The temperature-dependent gas properties for Reynolds number, Re, and Prandtl number, Pr, are taken as those of air from Ref. [16]. The gas velocity is assumed to be equal to $2 \mathrm{~m} / \mathrm{s}$. The corrected temperature is relatively insensitive to gas velocities between $1 \mathrm{~m} / \mathrm{s}$ and $3 \mathrm{~m} / \mathrm{s}$, consistent with the results of Shaddix [12].

The uncertainty of Eq. (2) is discussed in detail in Ref. [17]. This correction formula is included for the convenience of mathematical modeling. 


\subsection{Centerline Velocity Measurements}

A bi-directional probe is located above the burner centerline and moved using a computer controlled translation device. A full description of the measurement and data processing methods is given in Ref. [11]. The pressure difference between the front and rear side of the probe (16 mm outer diameter and $2.5 \mathrm{~cm}$ long) is measured with multiple pressure transducers with various instrument response times. The gas velocity, $V_{\mathrm{g}}(t)$, can be determined from the corrected pressure difference between the front and rear sides of the probe, $\Delta P_{c}(t)$, and the local gas density, $\rho(t)$ :

$$
V_{\mathrm{g}}(t)=\frac{1}{k_{p}(t)} \sqrt{\frac{2 \Delta P_{c}(t)}{\rho(t)}}
$$

The gas density is calculated from the gas temperature, $T_{\mathrm{g}}(t)$, which is measured near the probe. The parameter $k_{p}(t)$ is the probe constant, whose value depends on several factors including the Reynolds number, probe shape, and flow approach angle. The temperaturedependent gas properties are taken as those of air. As many as three transducers are used for

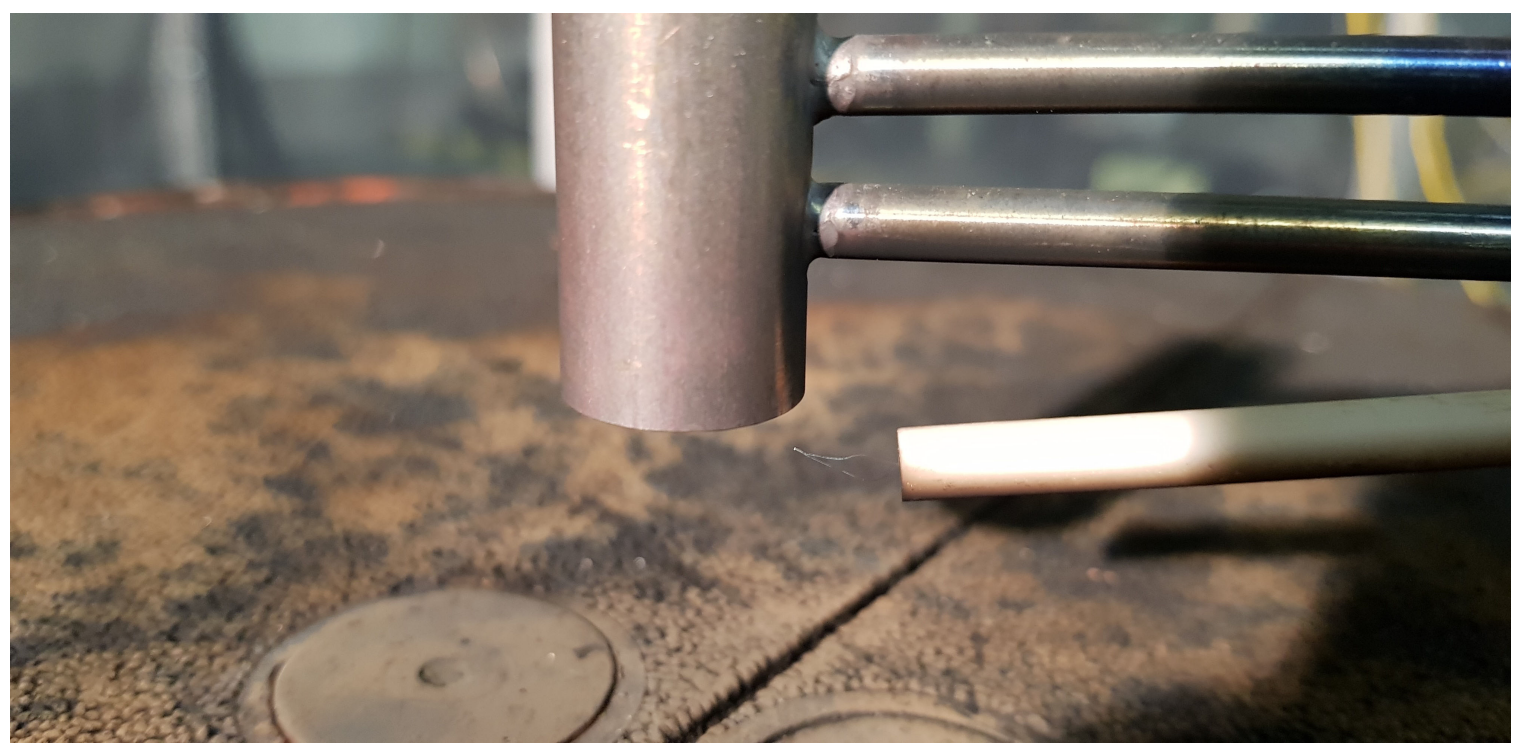

Fig. 4. Photograph of a bi-directional probe with a Type $S, 25 \mu \mathrm{m}$ wire diameter, bare-bead thermocouple positioned a few $\mathrm{cm}$ above the water-cooled, sintered-metal, $37 \mathrm{~cm}$ diameter, gas burner.

any single time series pressure measurement. Pressure and temperature measurements are typically made twice. A Type $\mathrm{S}(\mathrm{Pt} 10 \% \mathrm{Rh} / \mathrm{Pt}$ ), $25 \mu \mathrm{m}$ diameter, bare-wire, thermocouple is positioned $5 \mathrm{~mm}$ upstream of the probe as seen in Figure 4. The thermocouple bead is nearly spherical with a bead diameter of approximately $125 \mu \mathrm{m}$ as determined using optical microscopy. Voltage signals from the pressure transducers and the Type $S$ thermocouple are obtained using a DAQ (Model: SCXI-1600, National instrument Inc). Data sampling 
rates are either $250 \mathrm{~Hz}$ or $500 \mathrm{~Hz}$, depending on the case. Data is acquired for $2 \mathrm{~min}$ at each position along the axial centerline above the burner.

The measured pressure difference, $\Delta P(t)$, is corrected to account for the instrument time response, which is treated in a manner similar to the inertia correction in a thermocouple measurement:

$$
\Delta P_{c}(t)=\Delta P(t)+\tau_{p} \frac{d(\Delta P(t))}{d t}
$$

The time derivative of the pressure difference is calculated using a second-order polynomial fit using three consecutive data points in the pressure difference time series. The parameter $\tau_{p}$ is the pressure transducer instrument response time. This parameter is experimentally determined for each transducer by applying a step-function pressure change and observing the transient signal response as discussed in Ref. [11].

\subsection{Measuring the Volume Fraction of Gas Species via GC/MS}

Figure 5 displays the flow diagram for gas and particulate sampling. The GC/MS is equipped with a $2 \mathrm{~mL}$ sample loop maintained at approximately $200{ }^{\circ} \mathrm{C}$. The gases are extracted by a vacuum pump located downstream of the GC/MS. Gas samples are collected using a thermal quenching probe composed of concentric, stainless-steel tubes with outer annular coolant flow and inner extracted sample flow. The outer and inner tube diameters are $16 \mathrm{~mm}$ and $8 \mathrm{~mm}$, respectively. Water at approximately $90^{\circ} \mathrm{C}$ flows through the sampling probe for the duration of the experiment. The remainder of the sampling line leading into the GC/MS is heated to approximately $140{ }^{\circ} \mathrm{C}$ with electrical heating tape to prevent condensation of water and other condensable species (e.g., methanol, ethanol, benzene, etc.).

Directly behind the sampling probe is a heated soot filter which is used to make a gravimetic soot measurement and eliminate soot from the gas sample injected into the GC. An in-line heated $150 \mathrm{~mL}$ chamber ensures that the sample is well-mixed and representative of the local sampling volume. Depending on the probe's lateral location within the fire, the sampling period varies from $12 \mathrm{~min}$ to $25 \mathrm{~min}$, ensuring a significant quantity of soot is collected to make a measurement is made and that the gases are representative of the sampling location and have completely swept through the sample loop. The sample flow is controlled using a mass flow controller (Alicat Scientific MC-Series, model no. MC2SLPM-D/5M, 5IN) located upstream of the vacuum pump in the sampling line. The mass flow controller is maintained at a constant pressure (approx. $90 \mathrm{kPa}$ ) and temperature (309 $\mathrm{K})$. The mass flow is equivalent to 0.190 SLPM of air. Since the extracted gas sample is well-mixed within the sample reservoir and because the mass flow controller is maintained at a constant temperature and pressure, the mass flow at the fire sample location is approximately constant. The transient gas density at the fire sample location, however, is inversely proportional to the local temperature in the fire. Conservation of mass requires that the velocity at the probe inlet will vary inversely proportional to the local density. Monitoring of the pressure is important to ensure that clogging of the soot filter does not occur, which would change the pressure at the mass flow controller and thereby the mass flow rate. This 


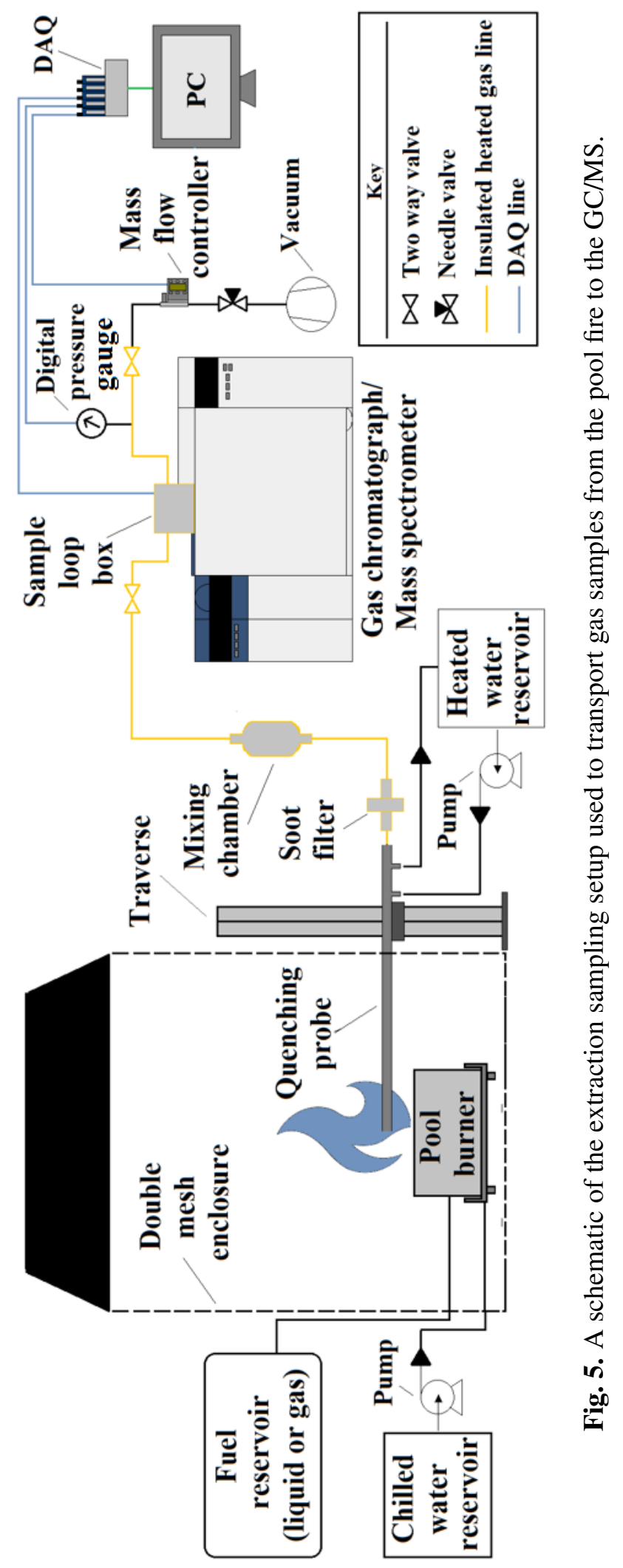


method provides an ensemble-averaged sample for analysis of composition. All measurements are replicated at least twice to provide an average concentration measurement.

Since the gas sample is taken for several minutes and well-mixed in the sample gas reservoir, the sample injected into the GC/MS for gas analysis represents a time-averaged extracted sample. In the turbulent fires investigated in this study, at any position, the temperature, and the density, varies as a function of time at a nominal frequency related to the dominant puffing frequency of the fire, which ranged from $2.3 \mathrm{~Hz}$ to $2.6 \mathrm{~Hz}$ for the fires considered here.

During the gas sampling procedure, the mass flow rate is recorded at $2.0 \mathrm{~Hz}$. The characteristic length of the regime of influence of the extractive gas sampling probe is estimated to range between $0.5 \mathrm{~cm} \pm 0.1 \mathrm{~cm}$ to $3.9 \mathrm{~cm} \pm 0.7 \mathrm{~cm}$. The characteristic length of the regime of influence is calculated using an approximation described in Appendix C. After the gas sampling period, two quarter-turn valves located on opposite ends of the sample loop are closed, at which point pressure measurements, obtained from a digital pressure gauge (OMEGA DPG409-030DWU), and temperature measurements, acquired by a K-type thermocouple located at the GC/MS sample loop injection port, are collected at $2.0 \mathrm{~Hz}$ for $50 \mathrm{~s}$ directly followed by sample injection.

Gas species measurements are made using an Agilent 5977E Series GC/MS. The GC/MS quantifies a variety of stable reactant, intermediate, and product species collected from the fire plume using helium as the carrier gas. The GC/MS utilizes an Agilent J\&W Select Permanent Gases $/ \mathrm{CO}_{2}$ High-Resolution column, which separates permanent gases (i.e., gases that are incapable of liquefaction). As shown in Fig. 6, the column is a parallel configuration consisting of a $25 \mathrm{~m}$ x $0.32 \mathrm{~mm}$ PoraBOND Q capillary column coupled with a $50 \mathrm{~m}$ x $0.53 \mathrm{~mm}$ Molsieve $5 \AA$ capillary column. The PoraBOND has high retention for several species, including carbon dioxide, but cannot completely separate oxygen, nitrogen, and other species resulting in a single composite peak. The Molsieve $5 \AA$ column includes zeolite-based materials with a pore size of $5 \AA$, allowing for separation and very high retention of permanent gases. Due to the multi-component setup of the column, some species (e.g., methane or propane) are observed to elute twice from the composite column causing two distinct peaks on the chromatogram.

The sample components elute from the column through a capillary tee splitter (Agilent G3184-60065), which divides the flow into two deactivated fused silica tubes (Agilent 1602615-10) of different lengths leading to the thermal conductivity (TCD) and mass selective (MS) detectors. The deactivated fused silica tubing flowing into the TCD and MS are cut to $1 \mathrm{~m}$ and $5 \mathrm{~m}$ to maintain carrier gas flows of approximately $3 \mathrm{~mL} / \mathrm{min}$ and $1 \mathrm{~mL} / \mathrm{min}$, respectively, such that the TCD and MS chromatograms are synchronized. Gas species are identified using the total ion chromatogram generated by the MS and quantified from the TCD's chromatogram.

For the ethanol, acetone, methane, and propane fires, the sample analysis time is approximately $60 \mathrm{~min}$, during which time the GC oven temperature is maintained at $30{ }^{\circ} \mathrm{C}$ for $10 \mathrm{~min}$, then ramped at $8^{\circ} \mathrm{C} / \mathrm{min}$ for 34 min until reaching a temperature of $300{ }^{\circ} \mathrm{C}$ which is maintained for the remainder of the analysis. For the methanol pool fires, the GC oven has 


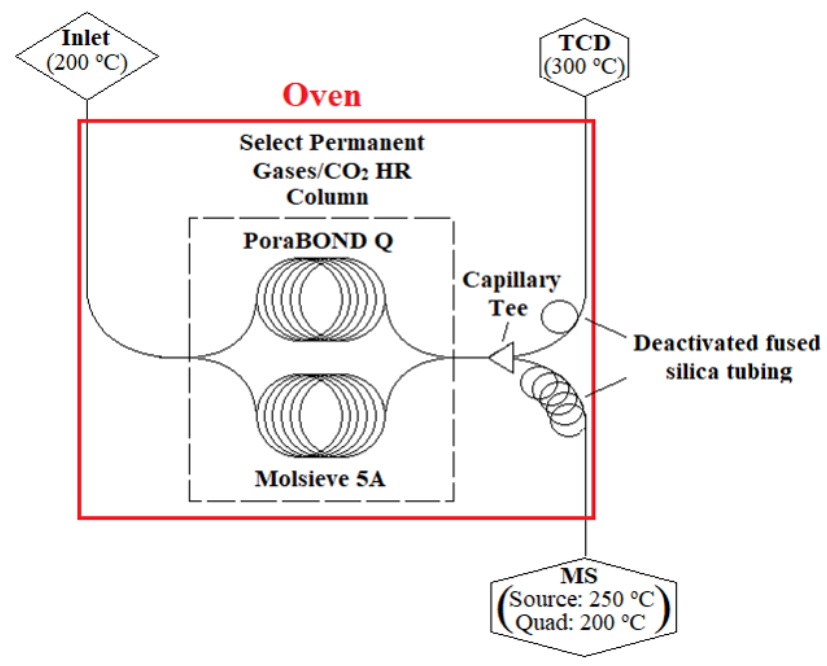

Fig. 6. An internal schematic of the GC detailing the column configuration and extension tubing feeding into the detectors.

the same setpoint temperature hold time and ramp rate, but only ramps to $192{ }^{\circ} \mathrm{C}$ to reduce overall analysis time. During the sample analysis, the TCD is maintained at $300{ }^{\circ} \mathrm{C}$ with a makeup and reference flow of $12 \mathrm{~mL} / \mathrm{min}$ and $27 \mathrm{~mL} / \mathrm{min}$, respectively. Additionally, the MS source and quad temperatures are $250{ }^{\circ} \mathrm{C}$ and $200{ }^{\circ} \mathrm{C}$, respectively, for the duration of the sample analysis.

A typical TCD chromatogram is shown in Fig. 7. The elution times for individual gas species are provided in Appendix D. The area associated with each peak is proportional to the number of moles of a particular species. Peaks are manually integrated using the Agilent software. Integration bounds of each peak are determined using the MS. In instances where peaks overlap, it is necessary to implement a tangential "skim" to improve integration resolution of an overlapping region, as shown in Fig. 7.

The area associated with a peak, $A$, is adjusted to account for the variation in the measured pressure, $P$, and temperature, $T$, of the sample:

$$
A_{\text {corr }}=A\left(\frac{P T_{\infty}}{P_{\infty} T}\right)
$$

The corrected area is converted into number of moles using a linear calibration curve determined using gas standards listed in Appendix F.2. The calibration functions of liquidvapors (e.g., water, methanol, ethanol, acetone) are obtained from a bubbler apparatus described in Appendix F.3.

Once the number of moles for all detected species is determined, the volume fraction of each species can be calculated. The mean volume fraction of a given species, $\bar{X}_{i}$, is the ratio of the number of moles of a given gas species, $n_{i}$, and the total number of moles identified by the GC/MS, $n_{\text {tot }}$, averaged over the repeated GC/MS gas injections. The total number of moles is determined from the summation of moles for each species quantified from the 


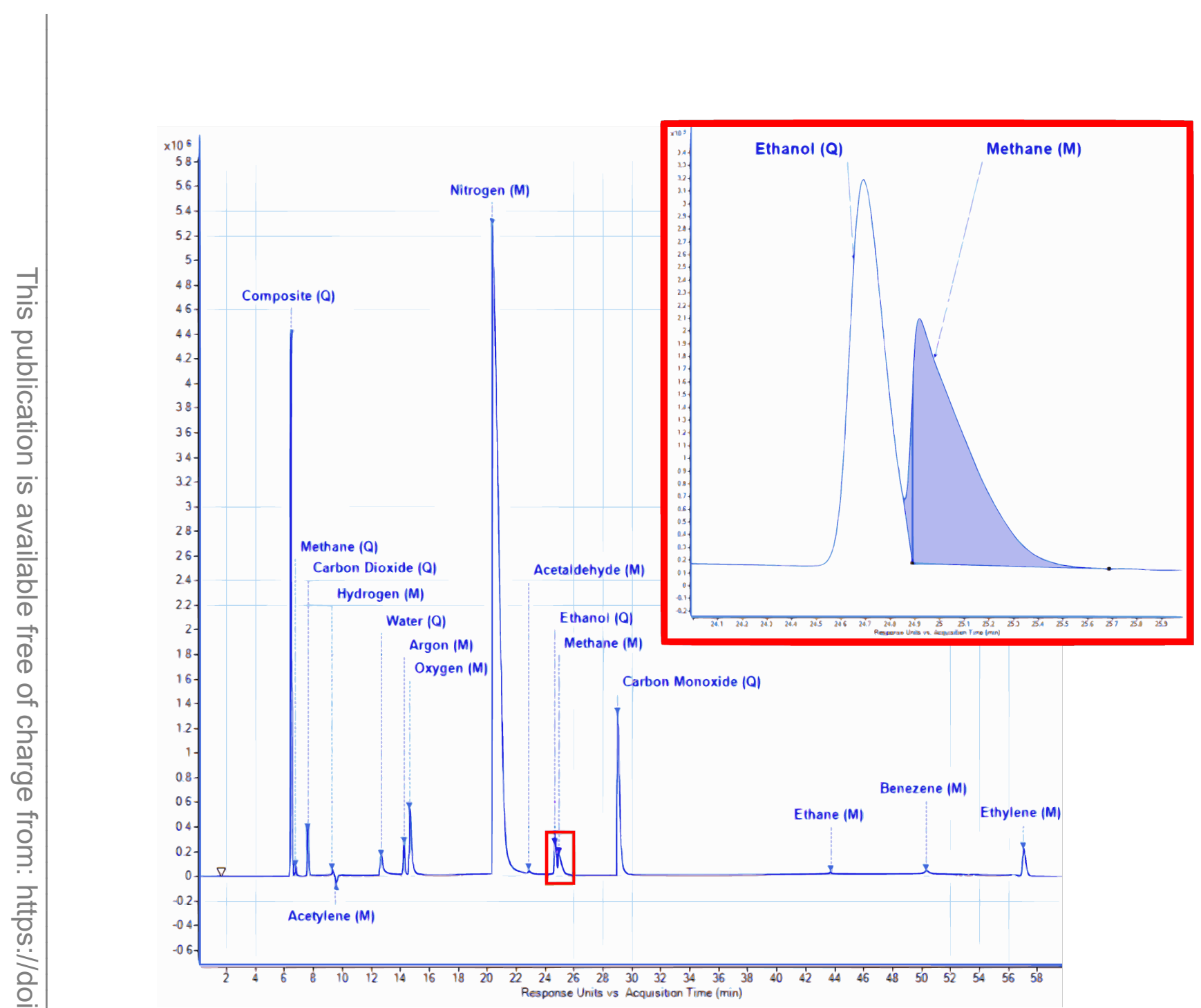

Fig. 7. TCD chromatogram of a pool fire gas sample and a magnified image of overlapping peaks with an example of tangential skim integration technique used to determine peak area

TCD chromatogram. In the case of water, the relative humidity measured within the room accounted for when calculating its volume fraction.

$$
\bar{X}_{i}=\frac{n_{i}}{n_{\mathrm{tot}}}
$$

The mean mass fraction, $\bar{Y}_{i}$, of a given species $i$ is calculated from the measured volume fraction, $\bar{X}_{i}$, using the following expression:

$$
\bar{Y}_{i}=\frac{\bar{X}_{i} \mathrm{~W}_{i}}{\sum \bar{X}_{i} \mathrm{~W}_{i}}=\frac{\bar{X}_{i} \mathrm{~W}_{i}}{W_{\mathrm{tot}}}
$$

where $\mathrm{W}_{i}$ is the molecular weight of a given species.

All measurements using the GC/MS are repeated at least twice at each location along the centerline of the pool fire. Gas species concentration measurements made at the same 
location are averaged. The variance in the volume fraction is a function of position and species. The uncertainty of the species measurements and the calibration procedure is discussed in Appendices E and F, respectively.

\subsection{Determining Soot Mass Fraction}

Soot mass fraction, $Y_{\mathrm{s}}$, is measured using a well established gravimetric technique [18]. Soot is filtered out of the gas stream using a stainless steel particulate filter holder (PALL 2220). Before an experiment, a desiccated $47 \mathrm{~mm}$ polytetrafluoroethylene (PTFE) filter is weighed and placed into its holder. The filter holder is positioned within the gas sampling line behind the quenching probe and heated with tape to approximately $140{ }^{\circ} \mathrm{C}$ to prevent condensation of water and liquid fuels on the filter. After sampling, the filter is removed and dried in a desiccator. After drying for $48 \mathrm{~h}$, the filter's final weight is measured. Approximately $1 \mathrm{mg}$ of soot is collected during the sampling period, which varies from $12 \mathrm{~min}$ to 25 min depending on the sampling location. The mass of the PTFE filter and cleaning patches are measured three times before and after each experiment ${ }^{2}$.

The soot mass fraction, $Y_{\mathrm{s}}$, is computed from the ratio of the mass of the soot collected from the PTFE filter and gun cleaning patches, $m_{\mathrm{s}}$, and the total mass of gas sampled, $m_{\mathrm{tot}}$, measured from the mass flow controller readings:

$$
Y_{s}=\frac{m_{\mathrm{s}}}{m_{\mathrm{tot}}}
$$

The total mass of gas sampled is the product of the average volumetric flow rate measured by the mass flow controller, $\dot{V}$, the density of the sample gas injected into the GC/MS, $\rho_{\text {gas }}$, the gas sampling time, $\Delta t$, and the ratio of the mass flow controller's temperature reading, $T_{\infty}$, to the effective temperature of the gas calculated from Eq. (2), $T_{\mathrm{g}}$.

$$
m_{\mathrm{tot}}=\dot{V} \rho_{g a s} \Delta t \frac{T_{\infty}}{T_{\mathrm{g}}}
$$

In Eq. (12), the density of the sample gas is determined from the total mass detected from the TCD and MS, $m_{\text {det }}$, for the injected sample volume, $V_{\mathrm{s}}$.

$$
\rho_{\mathrm{gas}}=\frac{m_{\mathrm{det}}}{V_{\mathrm{s}}}
$$

A description of the soot mass fraction uncertainty is provided in Appendix G.

\footnotetext{
${ }^{2}$ After some experiments, soot deposits are observed on the inner walls of the quenching probe. As shown in Fig. 8, dedicated gun cleaning patches (Hoppe's 9 1203S) are used to clean the inside of the quenching probe with no cleaning solvent. At least two patches are used to collect soot on the inside of the probe. A petri dish is placed below one end of the probe to catch dislodged soot and patches. Soot collection on the inside of the probe concludes once an applied patch is observed to have no soot. Patches are weighed before and $48 \mathrm{~h}$ after cleaning the inside of the probe
} 

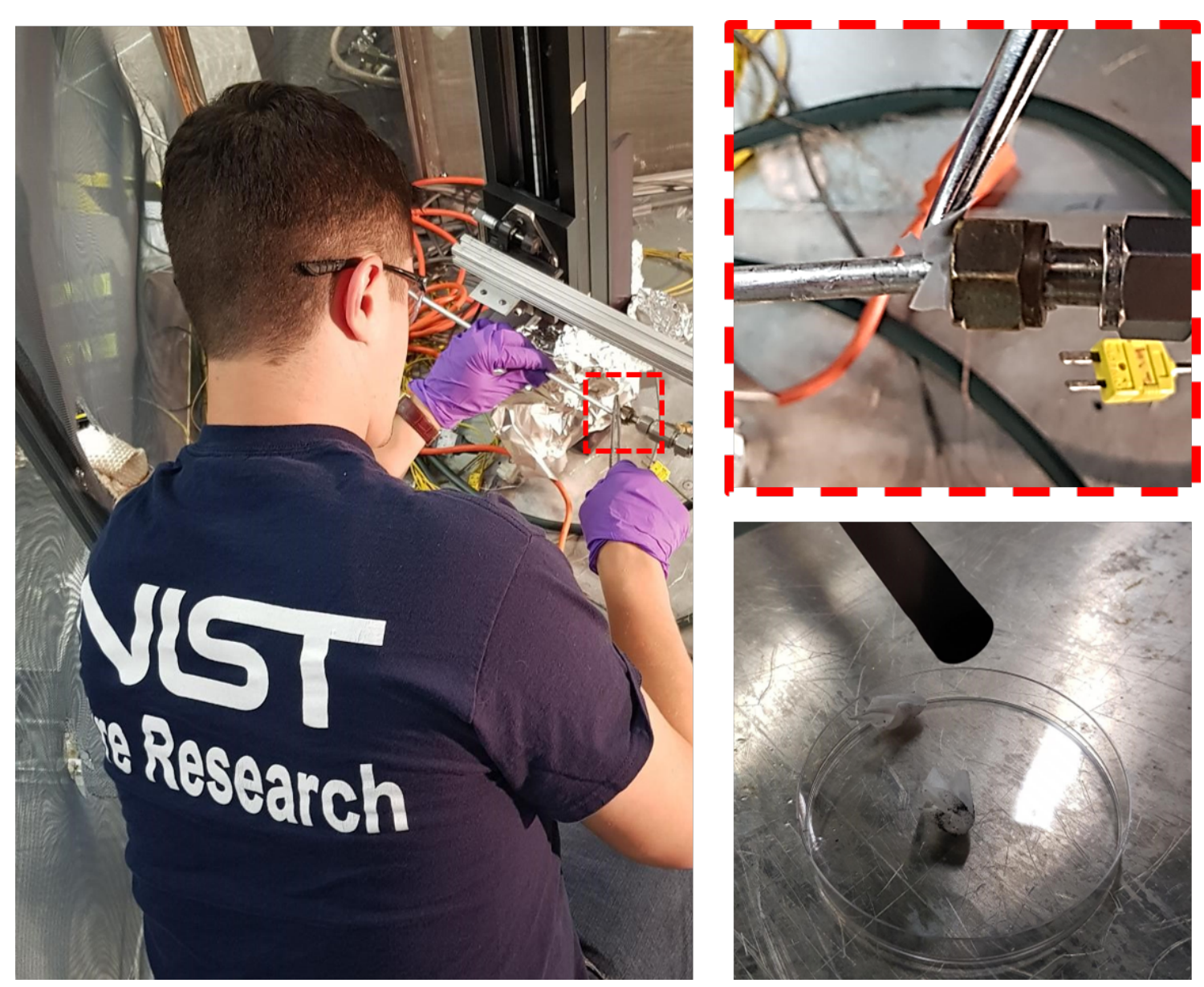

Fig. 8. Process of collecting soot from the internal walls of the quenching probe using gun cleaning patches 


\section{Results}

This section presents the flame height, temperature, gas species, and soot measurements made at incremental heights along the centerline of $30 \mathrm{~cm}$ diameter methanol, ethanol, and acetone pool fires and $37 \mathrm{~cm}$ diameter methane and propane fires.

\subsection{Flame Observations}

Figure 9 displays a series of snapshots depicting a single pulsation cycle of the methanol, ethanol, and acetone fires. The pulsation frequency is approximately $3 \mathrm{~Hz}$. The methanol fire is purely blue, whereas the ethanol, acetone, methane, and propane fires are more luminous and yellow. The measured time-averaged burning rates and calculated heat release rates are listed in Table 1. The heat release rates are calculated from Eq. (1).The methanol fire has the lowest average flame height, followed by the propane, ethanol, methane, and acetone. The measured mean flame heights match Heskestad's correlation [19] to within measurement uncertainty.

$$
\frac{L_{\mathrm{f}}}{D}=3.7\left(\dot{Q}^{*}\right)^{2 / 5}-1.02 \quad ; \quad \dot{Q}^{*}=\frac{\dot{Q}}{c_{p_{\text {air }}} \rho_{\infty_{\text {air }}} T_{\infty} \sqrt{g} D^{5 / 2}}
$$

Here, $D$ is the diameter of the pool fire $(30 \mathrm{~cm}), g$ is the acceleration of gravity, and $c_{p_{\text {air }}}$ and $\rho_{\infty_{\text {air }}}$ are the specific heat and the density of air at room temperature, $T_{\infty}$. The measured flame heights are also within the uncertainty bounds of measurements made by Kim et al. [7].

\subsection{Comparison of Pool Fires from Different Fuels}

Figure 10 displays the time-averaged, corrected gas temperatures as a function of the normalized vertical spatial coordinate, $z^{*}$ :

$$
z^{*}=\frac{z}{D^{*}} \quad ; \quad D^{*}=\left(\frac{\dot{Q}}{c_{p_{\text {air }}} \rho_{\infty_{\text {air }}} T_{\infty} \sqrt{g}}\right)^{\frac{2}{5}}
$$

Here, $z$ is the vertical spatial coordinate, $\dot{Q}$ is the heat release rate, $g$ is the acceleration of gravity, and $c_{p_{\text {air }}}$ and $\rho_{\infty_{\text {air }}}$ are the specific heat and the density of air at room temperature, $T_{\infty}$. The maximum mean temperature for each fuel peaks at approximately $z^{*}=0.2$. The maximum mean temperature for each fuel peaks is close to their respective stoichiometric values. The methanol temperature profile is in agreement with previous works [10]. The thermal inertia correction applied to the thermocouple temperature has less than a $5 \mathrm{~K}$ influence on the mean but significantly alters the RMS.

Figure 11 shows the mean and standard deviation of the upward gas velocity along the pool centerline as a function of $z^{*}$ (based on axial distance above the fuel surface) in the $30 \mathrm{~cm}$ diameter liquid pool fires and the $37 \mathrm{~cm}$ diameter gaseous pool fires. The results reported here complement those of Weckman [8] for the methanol fire, extending the 

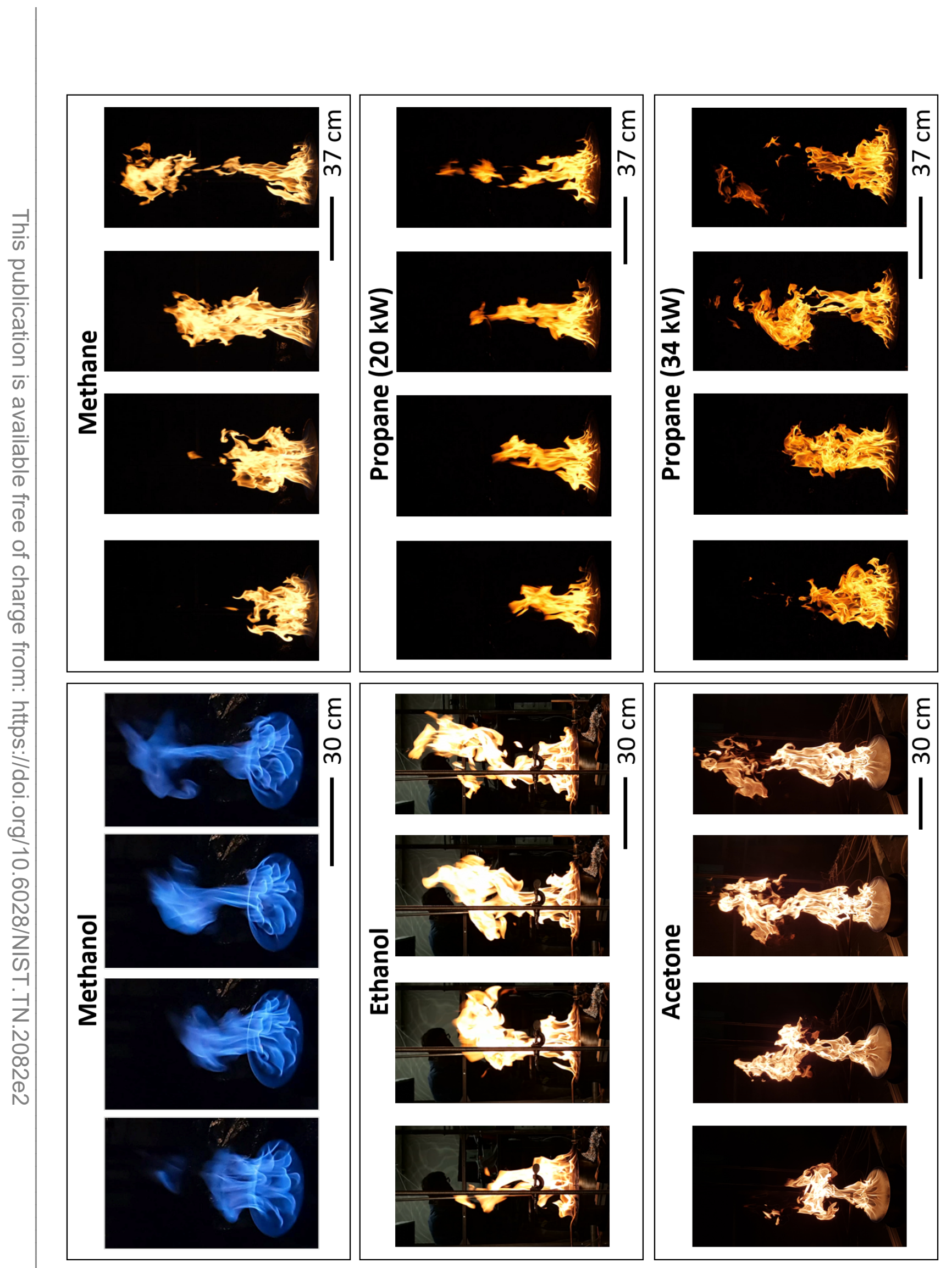

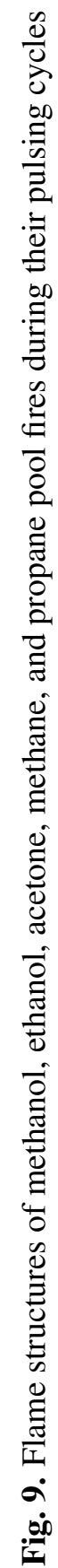
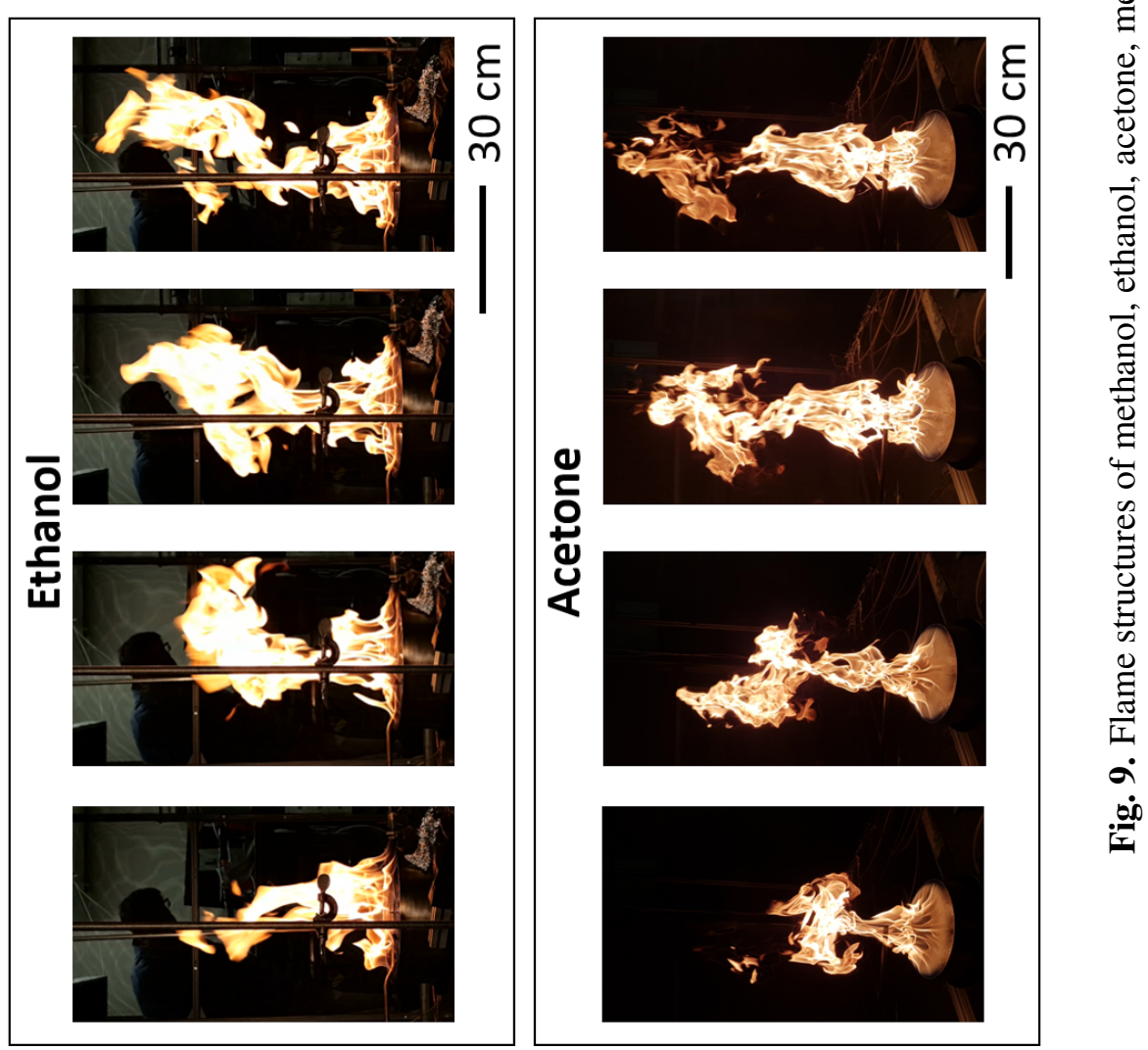
Table 1. List of measurements and thermochemical properties of fuels burning in a well-ventilated round $30 \mathrm{~cm}$ diameter and a $37 \mathrm{~cm}$ diameter pool fire burning in a quiescent environment. The uncertainty of the mass burning rate is discussed in Appendix A.1. The uncertainty of the heat release rate and $\dot{Q}^{*}$ is discussed in Appendix A.2.

\begin{tabular}{|c|c|c|c|c|c|c|}
\hline $\begin{array}{l}\text { Parameter } \\
\text { (units) }\end{array}$ & Methanol & Ethanol & Acetone & Methane & Propane & Propane \\
\hline $\begin{array}{l}\text { Mass } \\
\text { Burning } \\
\text { Flux }\left(\mathrm{g} / \mathrm{m}^{2} \mathrm{~s}\right)\end{array}$ & $12.4 \pm 1.1$ & $13.9 \pm 0.8$ & $17.6 \pm 2.7$ & $6.4 \pm 0.1$ & $4.2 \pm 0.1$ & $6.9 \pm 0.1$ \\
\hline $\begin{array}{l}\text { Ideal Heat } \\
\text { Release } \\
\text { Rate }(\mathrm{kW})\end{array}$ & $17.4 \pm 1.4$ & $26.3 \pm 1.5$ & $35.5 \pm 5.4$ & $34.5 \pm 0.5$ & $20.7 \pm 0.6$ & $34.4 \pm 0.6$ \\
\hline$\dot{Q}^{*}$ & $0.32 \pm 0.02$ & $0.48 \pm 0.01$ & $0.64 \pm 0.05$ & $0.37 \pm 0.01$ & $0.22 \pm 0.01$ & $0.37 \pm 0.01$ \\
\hline$D^{*}(\mathrm{~m})$ & $0.19 \pm 0.01$ & $0.22 \pm 0.01$ & $0.25 \pm 0.02$ & $0.25 \pm 0.01$ & $0.20 \pm 0.01$ & $0.25 \pm 0.01$ \\
\hline $\begin{array}{l}\text { Mean Flame } \\
\text { Height }(\mathrm{cm})\end{array}$ & $36.4 \pm 16.0$ & $61.1 \pm 28.2$ & $91.5 \pm 34.6$ & $64.0 \pm 31.0$ & $38.3 \pm 14.6$ & $50.0 \pm 16.0$ \\
\hline $\begin{array}{l}\Delta H_{c}(\mathrm{~kJ} / \mathrm{g})[20, \\
21]\end{array}$ & $19.90 \pm 0.1$ & $26.81 \pm 0.1$ & $28.56 \pm 0.1$ & $50.03 \pm 0.1$ & 46.34 & 46.34 \\
\hline $\mathrm{C} / \mathrm{H}$ Ratio & $1 / 4$ & $1 / 3$ & $1 / 2$ & $1 / 4$ & $3 / 8$ & $3 / 8$ \\
\hline
\end{tabular}

measurement range from one to four burner diameters above the fuel surface. The upward velocity along the centerline monotonically accelerates over the first diameter above the fuel surface. Above that, the velocity plateaus and slowly decreases with height. The velocity profiles for all fires are similar. The methanol fire has the lowest peak velocity. Details of the velocity measurement and its uncertainty are provided in Ref. [11].

Figure 12 displays the mean volume fraction of the major species, $\bar{X}_{i}$, as a function of $z^{*}$ for the methanol, ethanol, acetone, methane, and propane fires. Plots for individual species, including uncertainties, are displayed in Appendix H. Major species detected in the TCD and MS include combustion reactants (fuels and oxygen, $\mathrm{O}_{2}$ ), combustion products such as water, $\mathrm{H}_{2} \mathrm{O}$, and carbon dioxide, $\mathrm{CO}_{2}$, combustion intermediates such as carbon monoxide, $\mathrm{CO}$, hydrogen, $\mathrm{H}_{2}$, and inert gases such as nitrogen, $\mathrm{N}_{2}$, and argon, Ar. Methane is detected and quantified in all fires. In the case of the ethanol, acetone, methane, and propane fires, soot, benzene, acetylene, ethylene, and ethane are also detected and quantified. Trace amounts of other species are also detected including propene, acetaldehyde, and ethyl acetate, consistent with previous literature [22, 23]. 


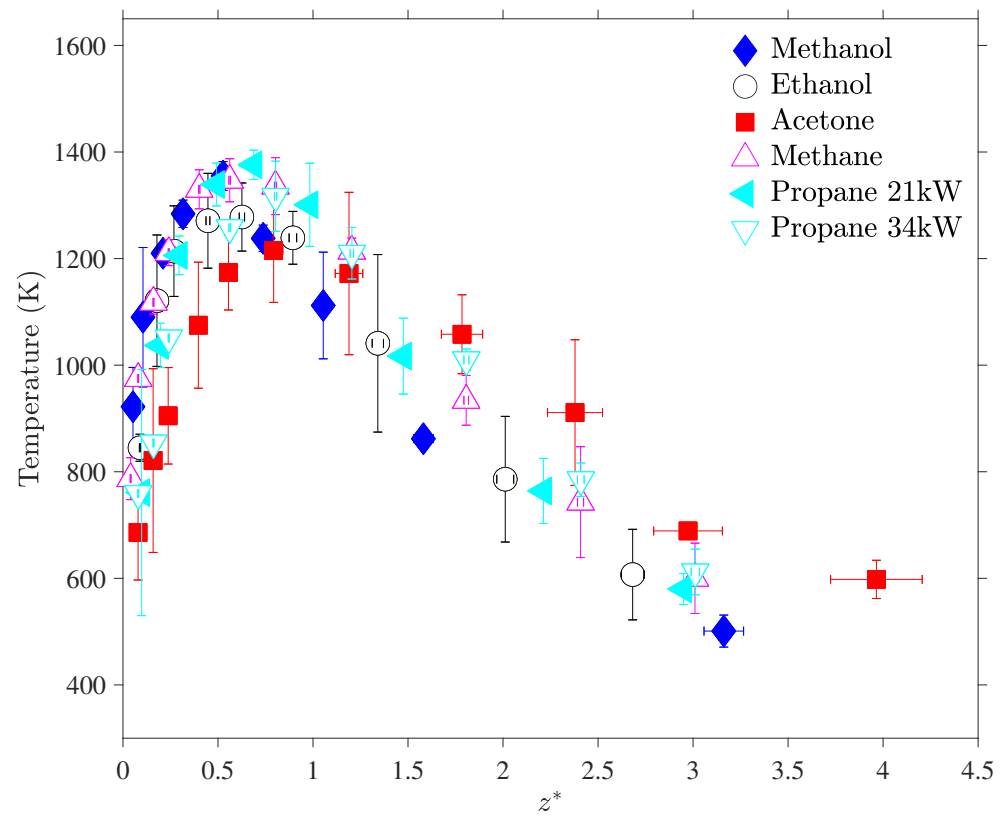

Fig. 10. Mean centerline gas temperature profiles of methanol, ethanol, acetone, methane, and propane pool fires during their pulsing cycles as a function of $z^{*}$. Vertical error bars represent the combined uncertainty of the measurement with a coverage factor of 2 . Horizontal error bars represent the combined uncertainty estimated from the law of propagation of uncertainty.

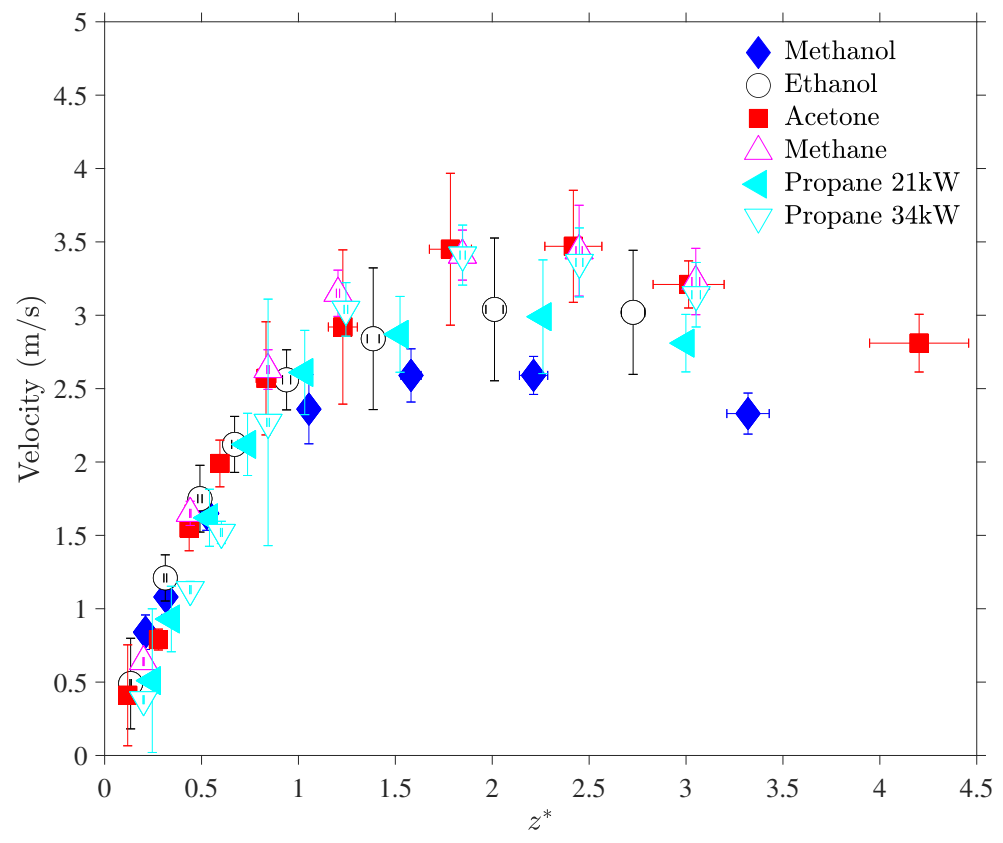

Fig. 11. Mean centerline velocity profiles of methanol, ethanol, acetone, methane, and propane pool fires during their pulsing cycles as a function of $z^{*}$. Vertical error bars represent the combined uncertainty of the measurement with a coverage factor of 2 . Horizontal error bars represent the combined uncertainty estimated from the law of propagation of uncertainty. 

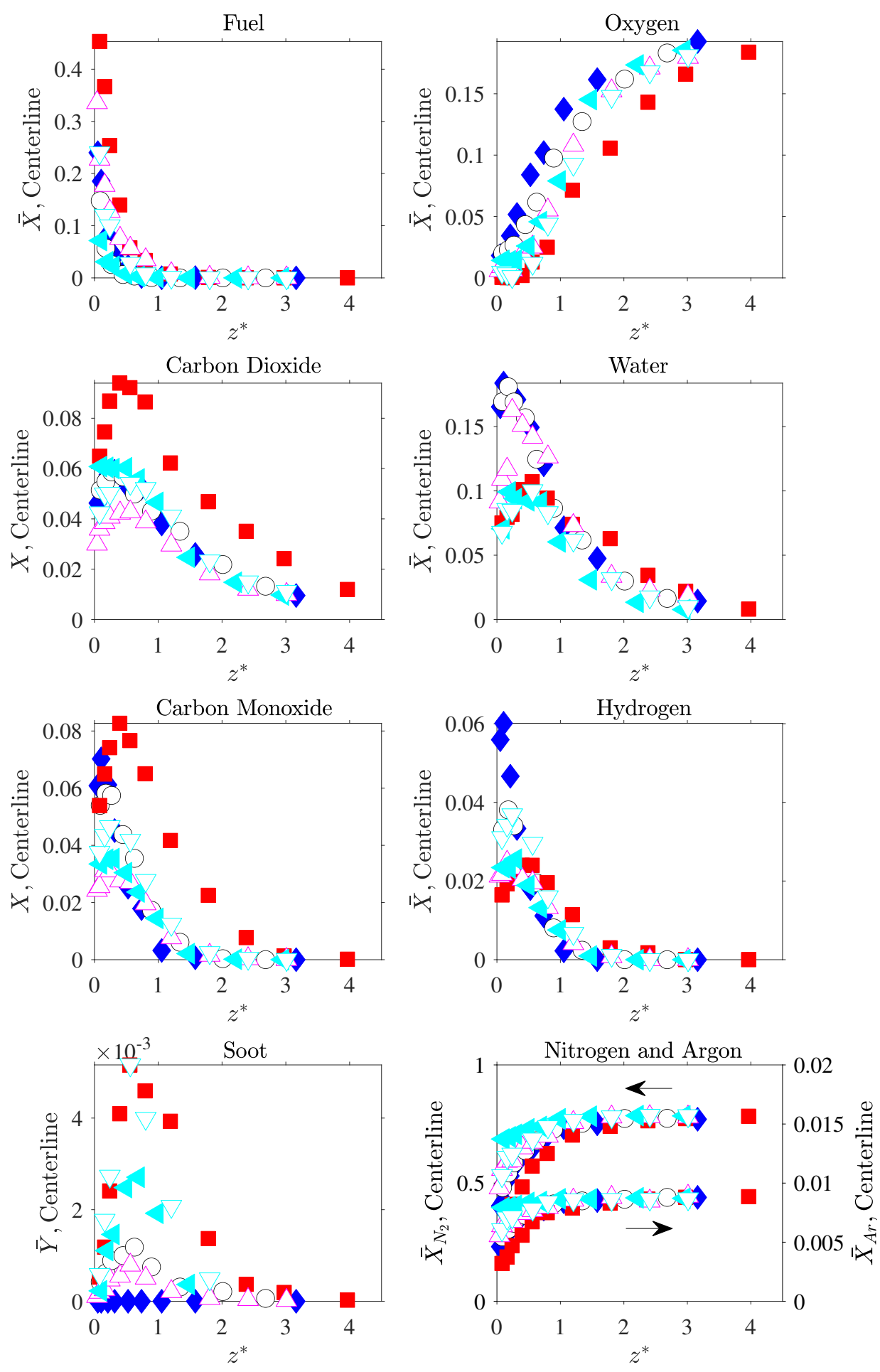

Fig. 12. Centerline volume fraction and soot mass fraction profiles of methanol $(\downarrow)$, ethanol $(\bigcirc)$, acetone $(\mathbb{\square})$, methane $(\triangle), 21 \mathrm{~kW}$ propane $(\triangleleft)$, and $34 \mathrm{~kW}$ propane $(\nabla)$ pool fires as a function of $z^{*}$ 


\section{Verifying Gas Species Measurements}

This section presents several different techniques of verifying the accuracy of the species concentration measurements.

\subsection{Counting Moles}

As a way to verify the accuracy of the experimental method, specifically the calibration procedure and curve fit, the total moles, $n_{\text {tot }}$, identified by the TCD and MS is compared to the total moles injected into the GC/MS system, $n_{\mathrm{inj}}$, which is calculated from the ideal gas law:

$$
n_{\mathrm{inj}}=\frac{P V_{\mathrm{s}}}{R T}
$$

Here, $R=8.314 \mathrm{~J} /(\mathrm{mol} \cdot \mathrm{K})$ is the universal gas constant, $V_{\mathrm{s}}=2 \times 10^{-6} \mathrm{~m}^{3}$ is the injected sample volume, and $P(\mathrm{~Pa})$ and $T(\mathrm{~K})$ are the mean pressure and temperature, respectively, collected before injecting the gas sample into the GC/MS as described in Section 2.6. The combined uncertainty of the total moles injected is detailed in Section F.1.

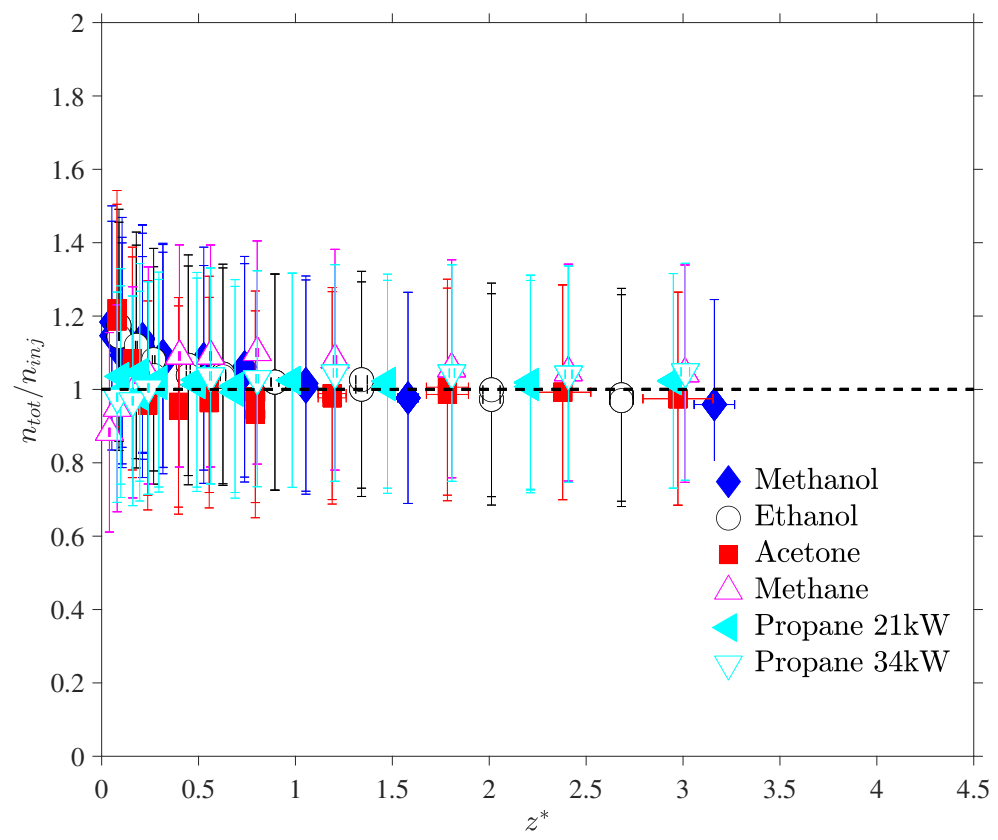

Fig. 13. Ratio of moles identified to moles injected, with uncertainty, as a function of $z^{*}$. Error bars represent the combined uncertainty estimated from the law of propagation of uncertainty. The uncertainty of the ratio is defined in in Section J.1.

Figure 13 shows the ratio of the moles identified by the TCD and MS to the moles injected into the GC/MS as a function of $z^{*}$. In most cases, the ratio is close to unity, indicating that the total moles injected into the GC/MS are all accounted for in the calculation. For low values of $z^{*}$, the ratio is higher than unity, indicating an error in the calibration 
which is most likely due to an over-prediction of the fuel species that are found at high concentrations near the fuel surface. Nevertheless, the expected values and estimated results are within experimental uncertainty, validating the calibration process of all detected species.

\subsection{Carbon to Hydrogen Ratio}

Another way to verify the accuracy of the gas species measurements is to calculate the ratio of carbon to hydrogen atoms contained in all gas species at each vertical measurement location:

$$
\frac{\mathrm{C}}{\mathrm{H}}=\frac{\sum \mathrm{x}_{i} \bar{X}_{i}}{\sum \mathrm{y}_{i} \bar{X}_{i}}
$$

where the summation is over all measured gas species, and $\mathrm{x}_{i}$ and $\mathrm{y}_{i}$ are the numbers of carbon and hydrogen atoms in the molecule, respectively. The carbon to hydrogen ratio of the fuel molecules are reported in Table 1, and the ratio for each fuel is shown in Fig. 14.

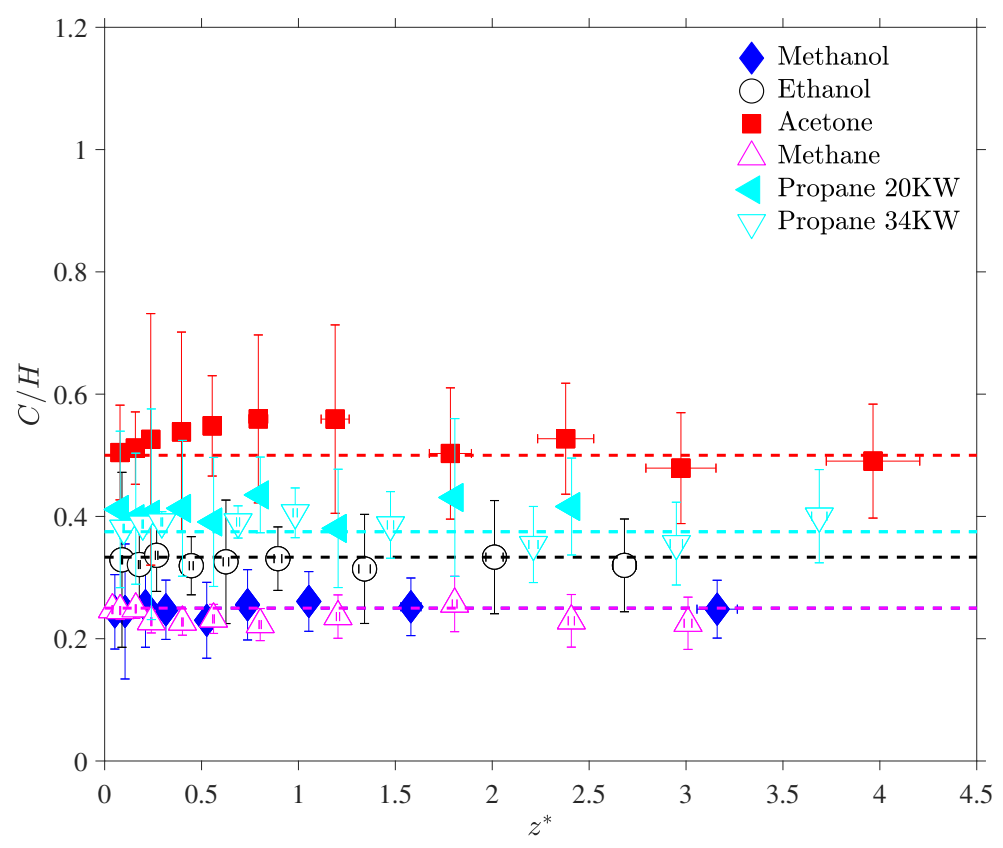

Fig. 14. Carbon to hydrogen ratio calculated from all measured gas species compared to the theoretical values as a function of $z^{*}$. Error bars represent the combined uncertainty estimated from the law of propagation of uncertainty

.The uncertainty of the ratio is defined in in Section J.2.

Figure 14 shows the comparison between the hydrogen to carbon ratio of the parent fuel, indicated by the dotted lines, and the calculated ratio at various positions within the centerline of the fires as a function of $z^{*}$. In all cases, the calculated $\mathrm{C} / \mathrm{H}$ is within the experimental uncertainty of the parent fuels' ratios. In several cases, the expected and calculated values are nearly matching, indicating that the concentration measurements of 
species consisting of carbon and hydrogen are accurately estimated using the experimental method. The consistency of the $\mathrm{C} / \mathrm{H}$ at different positions along the centerline of the fires also suggests that each of the extracted gas samples are well-mixed, as expected, showing no uneven distribution of carbon or hydrogen atoms when measured throughout the fire.

Another verification scheme considers the inert species argon and nitrogen whose molar ratio should be the same regardless of fuel type. The measured $\mathrm{Ar} / \mathrm{N}_{2}$ ratio in ambient air is $0.0117 \pm 0.0005$, and the measured ratio at different vertical locations within the fires is depicted in Fig. 15. All points fall within the uncertainty bounds of the ambient air measurement, indicating that measurements of the concentrations of argon and nitrogen are consistent throughout the fire. As with $\mathrm{C} / \mathrm{H}$, the consistency of the inert gas ratio suggests the extracted sample is well-mixed, further supporting the validity of the experimental methods.

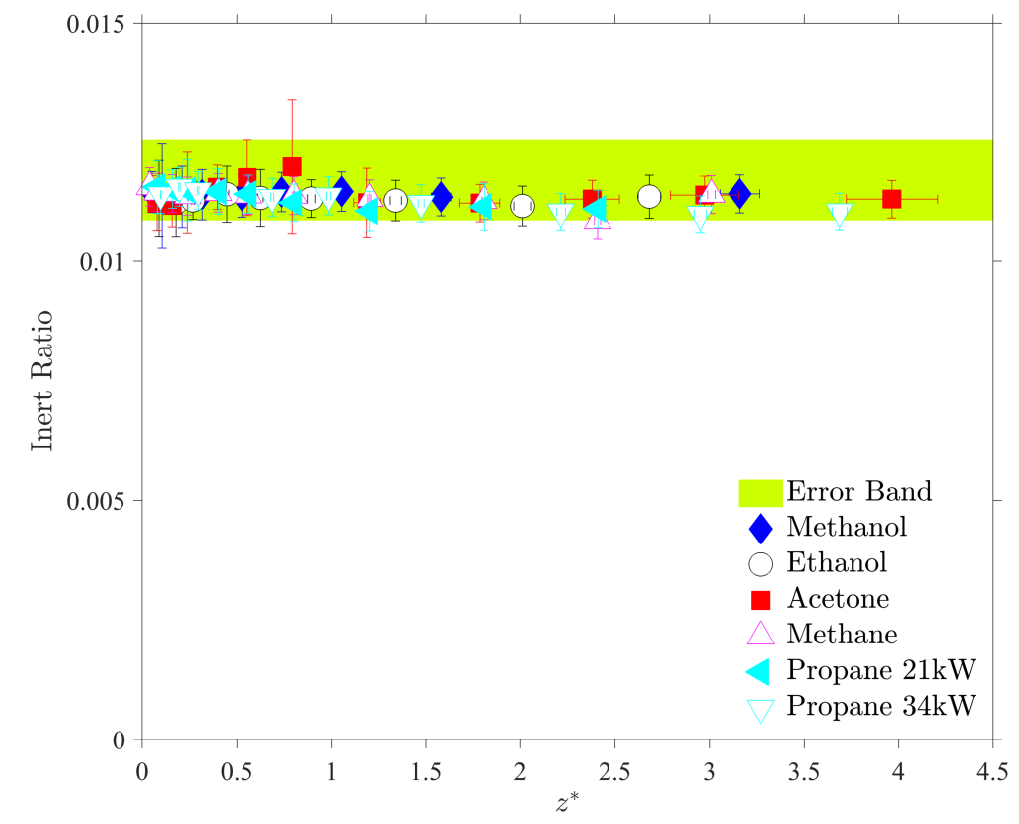

Fig. 15. Ar/ $\mathrm{N}_{2}$ molar ratio at different elevations compared to the uncertainty bounds of the measurement in ambient air as a function of $z^{*}$. Error bars represent the combined uncertainty estimated from the law of propagation of uncertainty. The uncertainty of the ratio is defined in in Section J.3. 


\subsection{Plotting the Results in Mixture Fraction Space}

The mixture fraction, $Z$, is defined as the mass fraction of the gases containing carbon, in addition to soot, that originate in the fuel stream. It can be expressed ${ }^{3}$ as follows:

$$
Z=\bar{Y}_{\mathrm{F}}+\frac{W_{\mathrm{F}}}{\mathrm{x}} \sum_{i \neq \mathrm{F}} \frac{\bar{Y}_{i}}{W_{i}}
$$

where $\bar{Y}_{\mathrm{F}}, W_{\mathrm{F}}$, and $\mathrm{x}$ are the mass fraction, molecular weight, and number of carbon atoms in the fuel molecule, respectively. Assuming ideal (i.e. no CO or soot), infinitely-fast (fuel and oxygen from the air cannot co-exist) combustion, the mass fractions of all species can be expressed as piece-wise linear "state relations" according to the following reaction:

$$
\begin{aligned}
\mathrm{C}_{\mathrm{x}} \mathrm{H}_{\mathrm{y}} \mathrm{O}_{\mathrm{z}} & +\eta\left(\mathrm{x}+\frac{\mathrm{y}}{4}-\frac{\mathrm{z}}{2}\right)\left(\mathrm{O}_{2}+3.76 \mathrm{~N}_{2}+0.0445 \mathrm{Ar}\right) \rightarrow \\
& \max (0,1-\eta) \mathrm{C}_{\mathrm{x}} \mathrm{H}_{\mathrm{y}} \mathrm{O}_{\mathrm{z}}+\max (0,1-\eta)\left(\mathrm{x}+\frac{\mathrm{y}}{4}-\frac{\mathrm{z}}{2}\right) \mathrm{O}_{2}+\min (1, \eta) \mathrm{x} \mathrm{CO}_{2}+ \\
& \min (1, \eta) \frac{\mathrm{y}}{2} \mathrm{H}_{2} \mathrm{O}+\eta\left(\mathrm{x}+\frac{\mathrm{y}}{4}-\frac{\mathrm{z}}{2}\right)\left(3.76 \mathrm{~N}_{2}+0.0445 \mathrm{Ar}\right)
\end{aligned}
$$

The parameter $\eta$ is the reciprocal of the local fuel equivalence ratio, $\phi$,

$$
\phi=\frac{(\mathrm{F} / \mathrm{A})}{(\mathrm{F} / \mathrm{A})_{\mathrm{st}}}=\frac{1}{\eta}
$$

where $\mathrm{F} / \mathrm{A}$ is the fuel-air mass ratio and the subscript st denotes the stoichiometric condition.

Figures 16, 17, 18, 19, 20, and 21 show the mean mass fraction measurements as a function of the mixture fraction for the methanol, ethanol, acetone, methane, and propane fires, respectively. The dotted lines represent ideal combustion calculated from Eq. (19). The vertical dotted line identifies the stoichiometric value of the mixture fraction. Additional plots that detail the averaged mass fractions of all detected species as a function of mixture fraction, with their expanded uncertainties, are provided in Appendix I.

Where the mixture fraction is much less than stoichiometric, all major gas species are in close agreement with the ideal state relations; the measured mass fractions of unburned fuel and $\mathrm{CO}$ are nearly zero, and the $\mathrm{O}_{2}$ is close to its respective theoretical value. The measured mass fraction of $\mathrm{CO}_{2}$ and $\mathrm{H}_{2} \mathrm{O}$ are found to peak close to the stoichiometric mixture fraction. In the fuel-rich region, the measured mass fraction of $\mathrm{CO}_{2}$ differs considerably from the ideal state relation due to the substantial portion of $\mathrm{CO}$ and soot.

\footnotetext{
${ }^{3}$ The uncertainty of the mixture fraction is determined from propagating the error in the mass fraction measurements. A detailed description of the mixture fraction uncertainty is provided in Appendix J.4.
} 


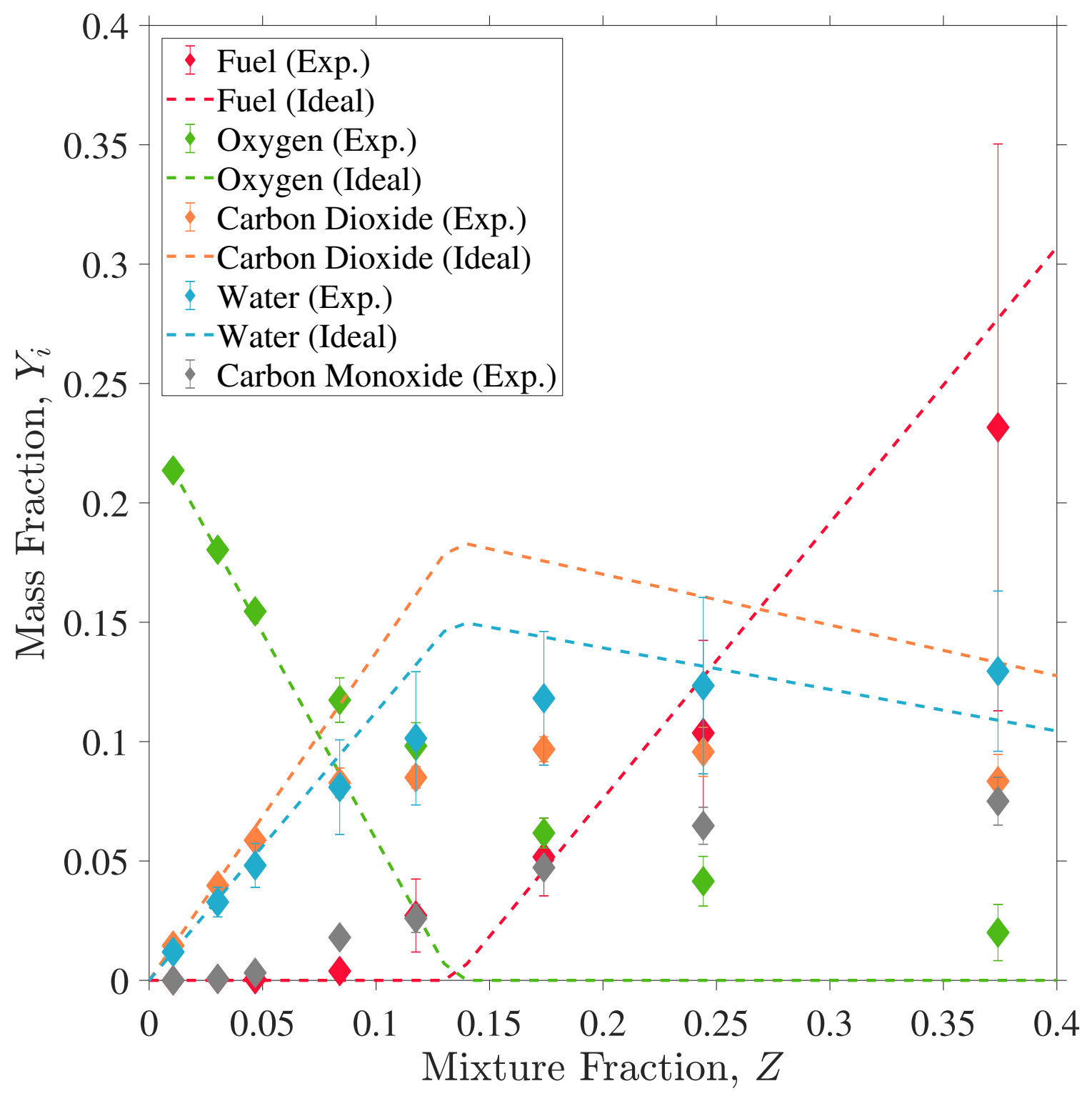

Fig. 16. Mean mass fractions as a function of mixture fraction, methanol. Error bars represent the combined uncertainty estimated from the law of propagation of uncertainty. The uncertainty of the mean mass fractions shown here is defined in Section E.2. 


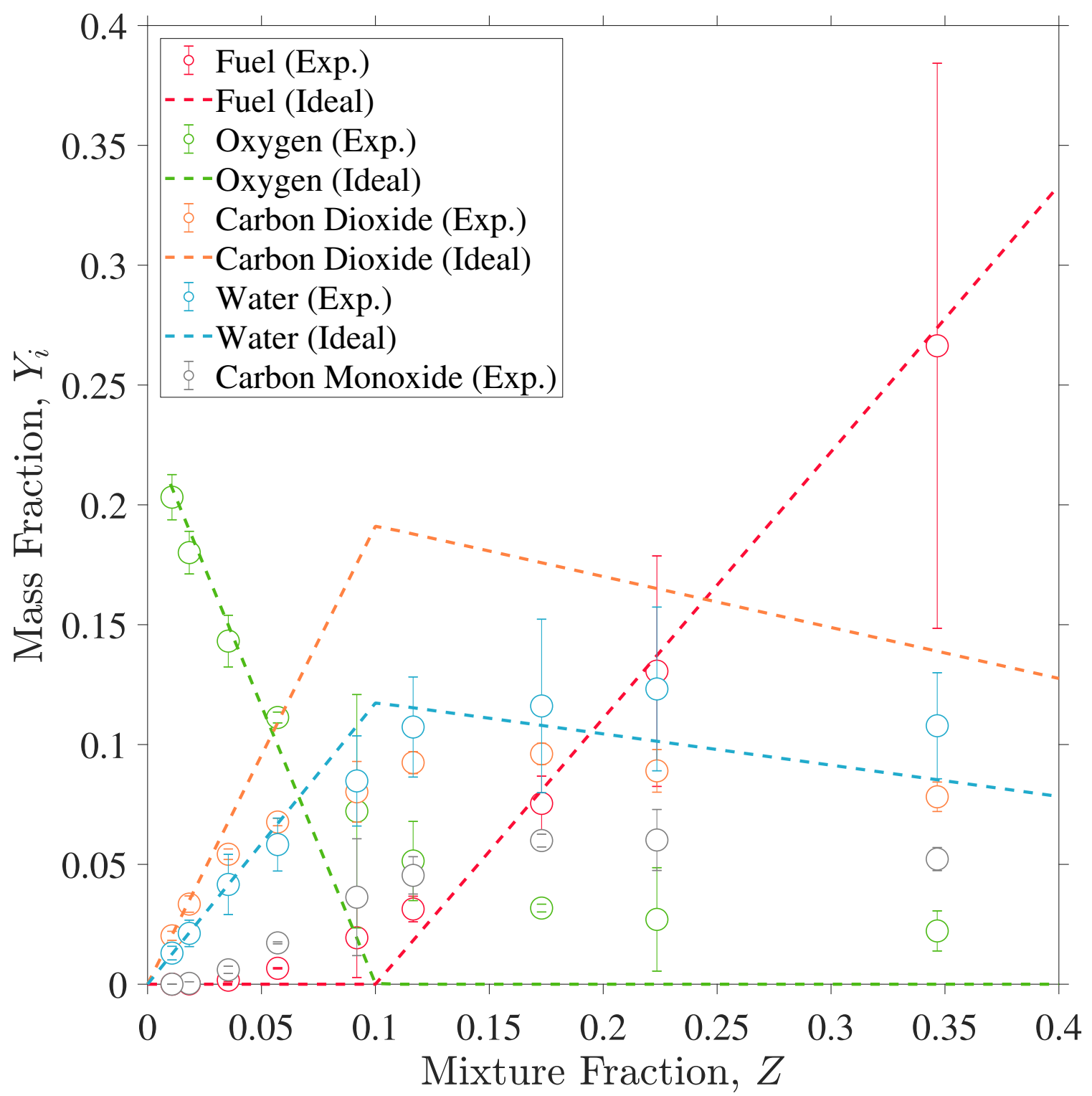

Fig. 17. Mean mass fractions as a function of mixture fraction, ethanol. Error bars represent the combined uncertainty estimated from the law of propagation of uncertainty. The uncertainty of the mean mass fractions shown here is defined in Section E.2. 


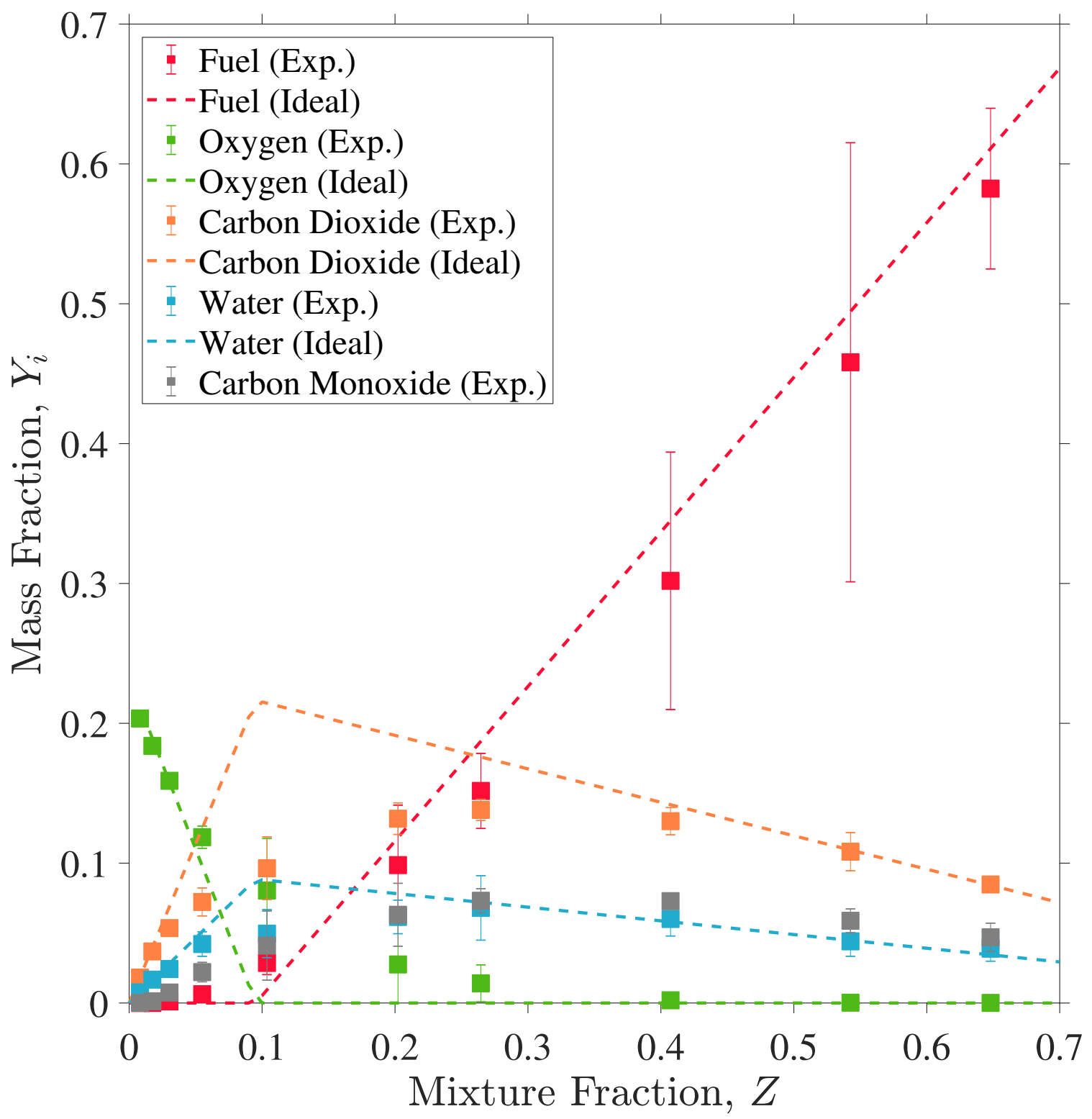

Fig. 18. Mean mass fractions as a function of mixture fraction, acetone. Error bars represent the combined uncertainty estimated from the law of propagation of uncertainty. The uncertainty of the mean mass fractions shown here is defined in Section E.2. 


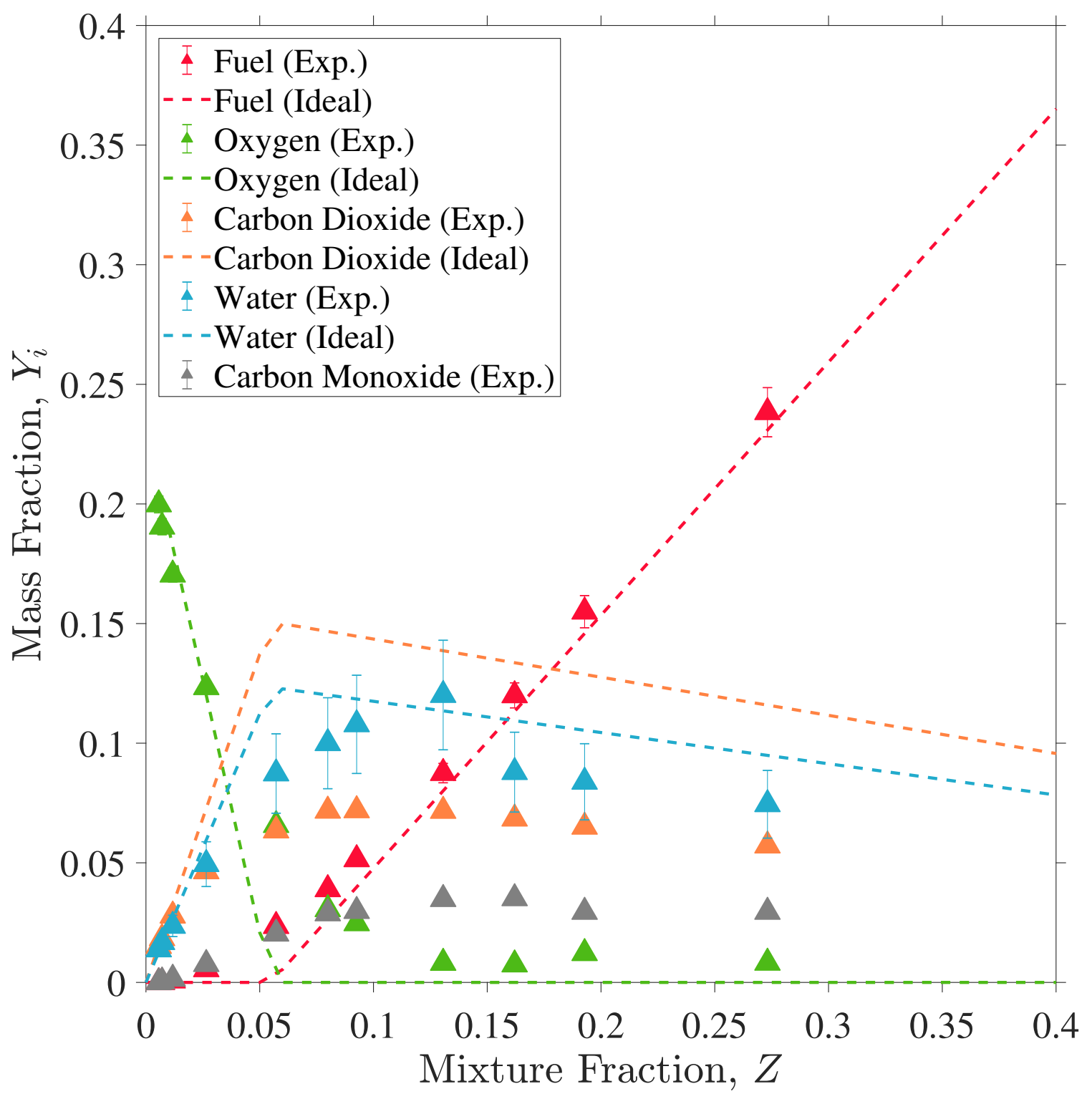

Fig. 19. Mean mass fractions as a function of mixture fraction, methane. Error bars represent the combined uncertainty estimated from the law of propagation of uncertainty. The uncertainty of the mean mass fractions shown here is defined in Section E.2. 


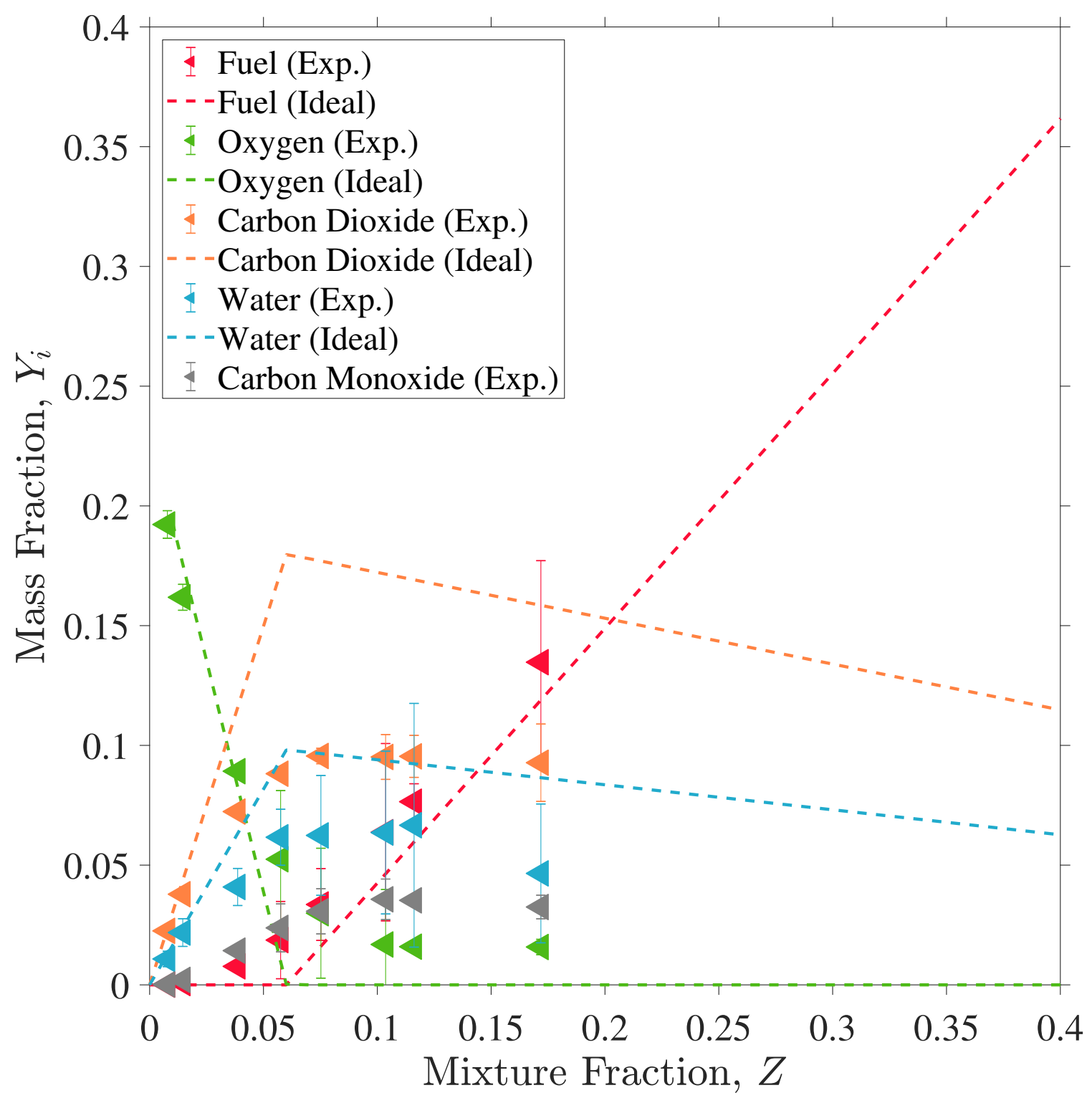

Fig. 20. Mean mass fractions as a function of mixture fraction, propane $(21 \mathrm{~kW})$. Error bars represent the combined uncertainty estimated from the law of propagation of uncertainty. The uncertainty of the mean mass fractions shown here is defined in Section E.2. 


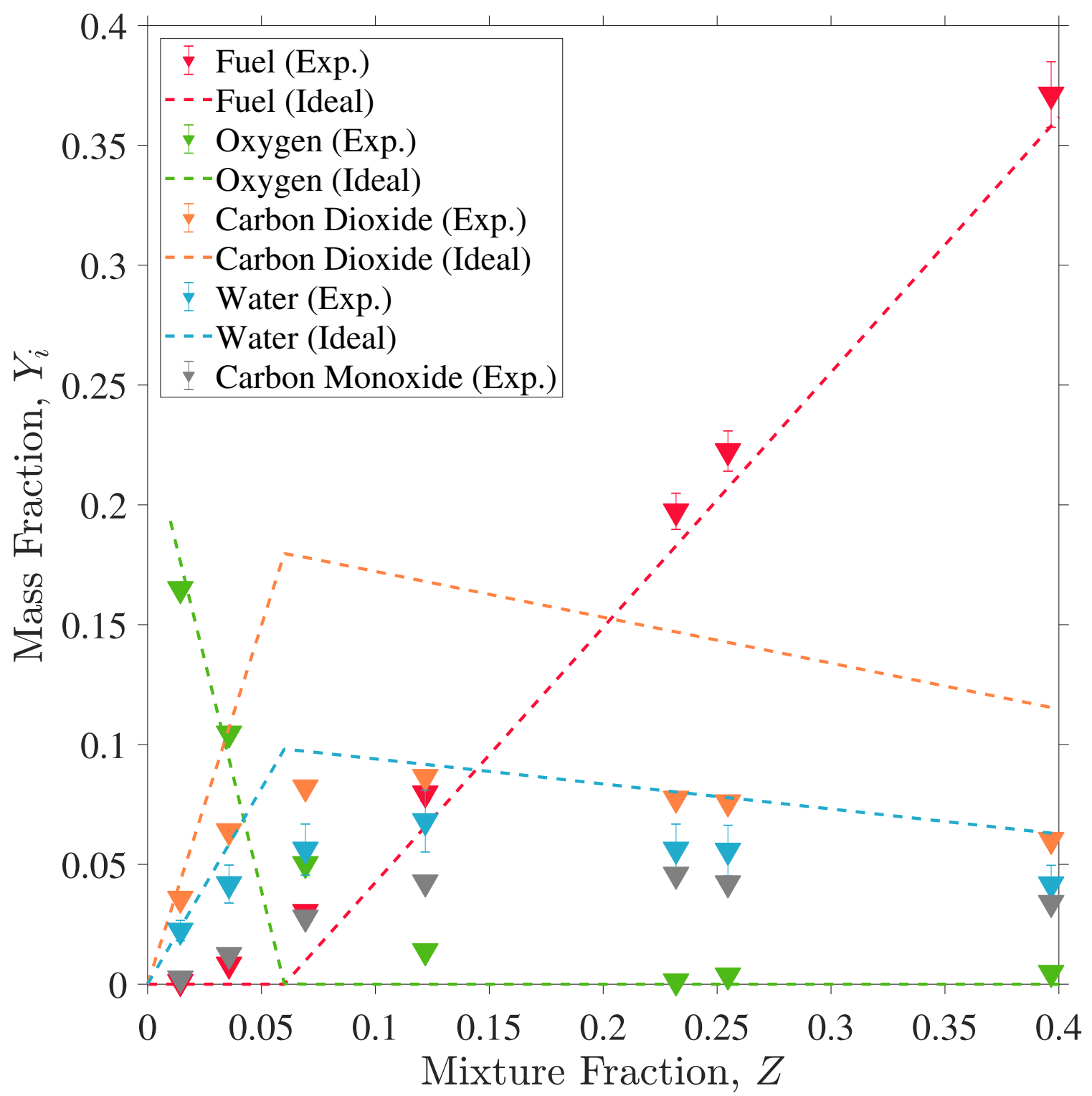

Fig. 21. Mean mass fractions as a function of mixture fraction, propane ( $34 \mathrm{~kW})$. Error bars represent the combined uncertainty estimated from the law of propagation of uncertainty. The uncertainty of the mean mass fractions shown here is defined in Section E.2. 


\section{Conclusion}

In summary, time-averaged local measurements of temperature, velocity, and gas species concentrations are made to characterize the structure of methanol, ethanol, and acetone $30 \mathrm{~cm}$ diameter pool fires and $37 \mathrm{~cm}$ effective diameter methane and propane pool fires steadily burning in a quiescent environment. A verification scheme is developed to verify the gas species measurements that considered the accuracy of the calibration and the overall stoichiometry of combustion for each fuel. The gas species measurements are favorably compared to the idealized mole, carbon to hydrogen, and inert ratios, which lends confidence to the veracity of the measurements. These local measurements complement previous measurements and provide insight into the complex chemical structure of medium-scale pool fires.

\section{Acknowledgments}

The authors would like to acknowledge Kevin McGrattan, who aided in the editing of this report. 


\section{References}

[1] S.J. Fischer, B. Hardouin-Duparc, and W.L. Grosshandler. The structure and radiation of an ethanol pool fire. Combustion and Flame, 70(3):291-306, Dec. 1987.

[2] A. Hamins and A. Lock. The Structure of a Moderate-Scale Methanol Pool Fire. NIST Technical Note 1928, National Institute of Standards and Technology, 2016.

[3] A. Hamins, S.J. Fischer, T. Kashiwagi, M.E. Klassen, and J.P. Gore. Heat feedback to the fuel surface in pool fires. Combustion Science and Technology, 97(1-3):37-62, 1993.

[4] A. Hamins, M. Klassen, J. Gore, and T. Kashiwagi. Estimate of flame radiance via a single location measurement in liquid pool fires. Combustion and Flame, 86(3):223228, 1991.

[5] A. Hamins, T. Kashiwagi, and R. Buch. Characteristics of pool fire burning. In Fire Resistance of Industrial Fluids. ASTM International, 1996.

[6] A. Lock, M. Bundy, E.L. Johnsson, A. Hamins, G.H. Ko, C.H. Hwang, P. Fuss, and R. Harris. Experimental Study of the Effects of Fuel Type, Fuel Distribution, and Vent Size on Full-Scale Underventilated Compartment Fires in an ISO 9705 Room. NIST Technical Note 1603, National Institute of Standards and Technology, 2008.

[7] S.C. Kim, K.Y. Lee, and A. Hamins. Energy balance in medium-scale methanol, ethanol, and acetone pool fires. Fire Safety Journal, 107:44-53, 2019.

[8] E.J. Weckman and A.B. Strong. Experimental investigation of the turbulence structure of medium-scale methanol pool fires. Combustion and Flame, 105(1):245-266, 1996.

[9] DIPPR ${ }^{\circledR}$. Design Institute for Physical Properties (DIPPR 801), January 2021. https://dippr.aiche.org/SampleDb.

[10] Z. Wang, W.C. Tam, K.Y. Lee, J. Chen, and A. Hamins. Thin filament pyrometry field measurements in a medium-scale pool fire. Fire Technology, September 2019.

[11] K. Sung, R. Falkenstein-Smith, and A Hamins. Velocity and Temperature Structure of Medium-Scale Pool Fires. NIST Technical Note 2162, National Institute of Standards and Technology, 2021.

[12] C.R. Shaddix. Correcting thermocouple measurements for radiation loss: a critical review. Technical Report CONF-990805, Sandia National Laboratories, 1999.

[13] Y.S. Touloukian, P.E. Liley, and S.C. Saxena. Thermophysical Properties of MatterThe TPRC Data Series. Volume 3. Thermal Conductivity-Nonmetallic Liquids and Gases. TEPIAC Technical Note ADA951937, Thermophysical and Electronic Properties Information Analysis Center, 1970.

[14] E.M. Wise, editor. The Platinum Metals and Their Alloys. Literary Licensing, LLC, 1 st edition, 2012.

[15] F.M. Jaeger and E. Rosenbohm. The exact formulae for the true and mean specific heats of platinum between $0^{\circ}$ and $1600^{\circ}$ C. Physica, 6(7-12):1123-1125, 1939.

[16] F.P. Incropera, A.S. Lavine, T.L. Bergman, and D.P. DeWitt. Fundamentals of Heat and Mass Transfer. Wiley, 2007.

[17] K. Sung, J. Chen, M. Bundy, M. Fernandez, and A. Hamins. The thermal character of 
a $1 \mathrm{~m}$ methanol pool fire. NIST Technical Note 2083, National Institute of Standards and Technology, 2020.

[18] M.Y. Choi, G.W. Mulholland, A. Hamins, and T. Kashiwagi. Comparisons of the soot volume fraction using gravimetric and light extinction techniques. Combustion and Flame, 102(1-2):161-169, 1995.

[19] G. Heskestad. Luminous heights of turbulent diffusion flames. Fire Safety Journal, 5(2):103-108, 1983.

[20] D.R. Burgess and A. Hamins. Heats of Combustion and Related Properties of Pure Substances. NIST Technical Note In Progress, National Institute of Standards and Technology, 2022.

[21] M.J. Hurley, editor. SFPE Handbook of Fire Protection Engineering. Springer, New York, 5th edition, 2016.

[22] S. Pichon, G. Black, N. Chaumeix, M. Yahyaoui, J.M. Simmie, H.J. Curran, and R. Donohue. The combustion chemistry of a fuel tracer: Measured flame speeds and ignition delays and a detailed chemical kinetic model for the oxidation of acetone. Combustion and Flame, 156(2):494-504, 2009.

[23] J. Gong, S. Zhang, Y. Cheng, Z. Huang, C. Tang, and J. Zhang. A comparative study of n-propanol, propanal, acetone, and propane combustion in laminar flames. Proceedings of the Combustion Institute, 35(1):795-801, 2015.

[24] M. Klassen and J.P. Gore. Structure and Radiation Properties of Pool Fires. NIST Technical Note GCR-94-651, National Institute of Standards and Technology, 1994.

[25] R. Buch, A. Hamins, K. Konishi, D. Mattingly, and T. Kashiwagi. Radiative emission fraction of pool fires burning silicone fluids. Combustion and Flame, 108:118-126, 1997.

[26] C.D.A. Hogben. Determination of the radiative properties of an acetone pool fire. $\mathrm{PhD}$ thesis, University of Waterloo, 1998.

[27] E.J. Weckman and A. Sobiesiak. The oscillatory behaviour of medium-scale pool fires. Symposium (International) on Combustion, 22(1):1299-1310, 1989.

[28] R.C. Corlett and T.M. Fu. Some recent experiments with pool fires. Pyrodynamics, 4(3):253-269, 1966.

[29] MaCFP measurement and computation of fire phenomena. https://github.com/ $\mathrm{MaCFP}$. 


\section{A. Uncertainty Analysis of Pool Fire Parameters}

\section{A.1 Mass Burning Rate}

For liquid fuels, the mean mass burning rate, $\dot{m}$, is determined by weighing the fuel reservoir at the start and end of the steady burning stage using a Precisa XB-6200C Precision balance, calibrated using a collection of standard weights. The Type A evaluation of uncertainty is the standard deviation in the measurements made during replicate experiments. The Type B evaluation of uncertainty is the reported accuracy of the balance, $0.01 \mathrm{~g}$, divided by the time interval. The Type A uncertainty dominates; thus, the standard uncertainty of liquid fuels, $u_{m}$, is approximately the standard deviation of the multiple measurements. For gaseous fuels, $\dot{m}$ is measured with a Brooks mass flow controller, Model 5863, configured for nitrogen. The Type A evaluation of uncertainty is the standard deviation in the measurements made during replicate experiments. The Type B evaluation of uncertainty of the controller is reported to be $1 \%$ of its full scale, $500 \mathrm{~L} / \mathrm{min}$. The Type B uncertainty dominates; thus, the standard uncertainty of gas fuel is approximately the bias uncertainty of the mass flow controller, $u_{\dot{m}}=0.01$.

\section{A.2 Heat Release Rate and $Q^{*}$}

The heat release rate, $\dot{Q}$, is the product of the fuel mass loss rate, $\dot{m}$, and the heat of combustion, $\Delta H$. The relative uncertainty of the former is far greater than the latter, thus:

$$
u_{\dot{Q}} \approx \Delta H u_{\dot{m}}
$$

The uncertainty of $Q^{*}$, is dominated by the relative uncertainty of $\dot{Q}$, thus:

$$
u_{Q^{*}} \approx \frac{u_{\dot{Q}}}{c_{p} \rho_{\infty} T_{\infty} \sqrt{g} D^{5 / 2}}
$$

\section{A.3 Mean Flame Height}

The mean flame height, $L_{\mathrm{f}}$, is determined using photographic analysis as described in Section 2. The Type A evaluation of uncertainty is the standard deviation of the height measurements, $s_{L_{\mathrm{f}}}$, made for each frame. The uncertainty of the distance measurement for each frame is relatively small, $0.1 \%$, and thus the standard uncertainty is approximately the standard deviation of the heights over multiple frames:

$$
u_{L_{\mathrm{f}}} \approx s_{L_{\mathrm{f}}}
$$

\section{A.4 Uncertainty Analysis of Temperature Measurements}

The uncertainty of the temperature measurements is estimated from Type A and Type B uncertainty. The Type A evaluation of uncertainty is the standard deviation of the corrected temperature measurements made at each flame location. The bias error sources, Type B 
uncertainty, in the S-type thermocouple, $u_{\text {inst }}$, used to measure temperature $\left(1.5^{\circ} \mathrm{C}\right)$ of the gas sample injected is relatively small compared to the Type A uncertainty.

\section{A.5 Uncertainty Analysis of Velocity Measurements}

The mean and standard deviation for the velocity is obtained from the multiple pressure transducers at a specific measurement position. The uncertainty associated with using the measurements from multiple pressure transducers contributes to the combined uncertainty of the gas velocity. Ref. [11] provides a detailed description of the velocity uncertainty methodology. 


\section{B. Figures of Time-Averaged Bead Temperature Readings}

\section{B.1 Methanol}

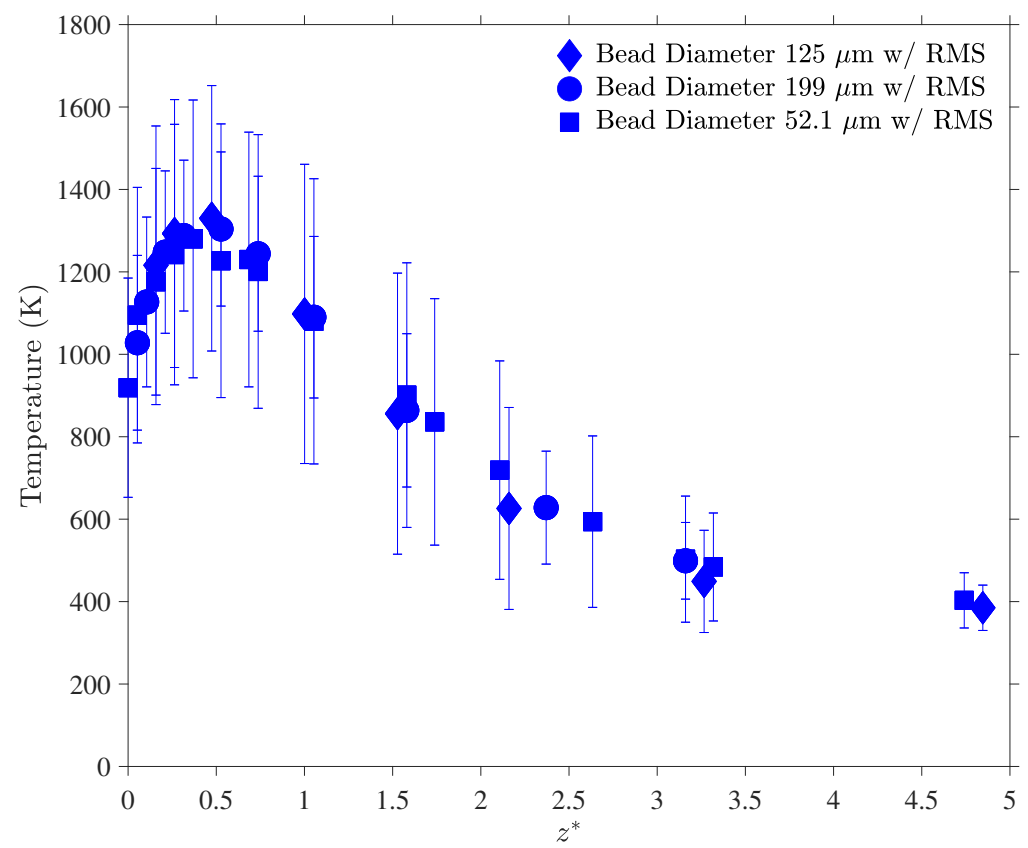

Fig. B.1. Mean and root mean square (RMS) centerline bead temperature profiles of methanol as a function of $z^{*}$ 


\section{B.2 Ethanol}

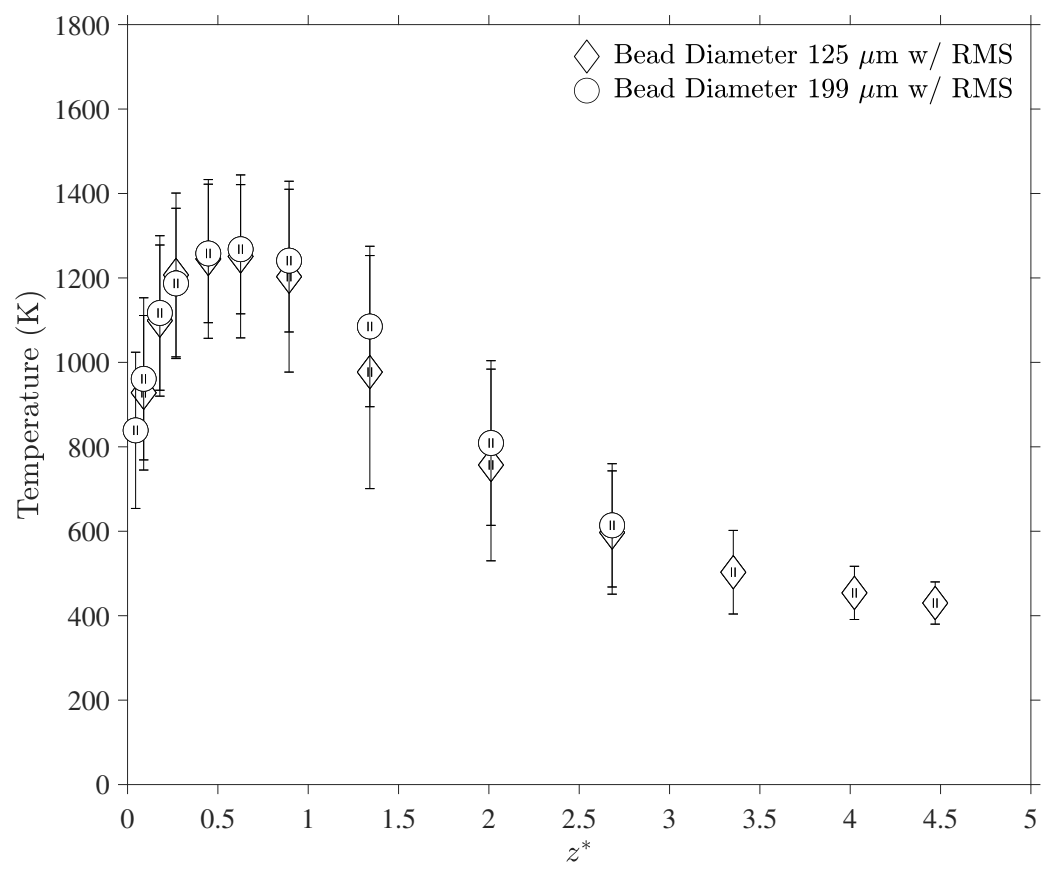

Fig. B.2. Mean and root mean square (RMS) centerline bead temperature profiles of ethanol as a function of $z^{*}$ 


\section{B.3 Acetone}

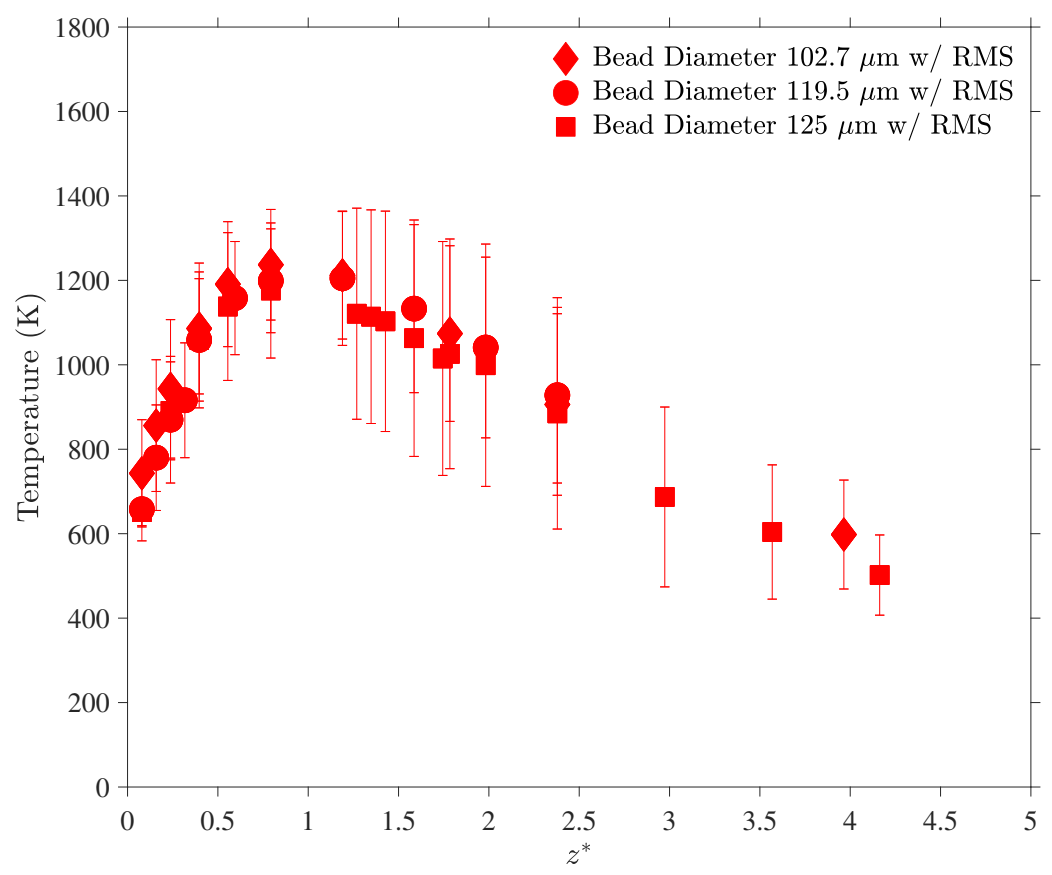

Fig. B.3. Mean and root mean square (RMS) centerline bead temperature profiles of acetone as a function of $z^{*}$ 


\section{B.4 Methane}

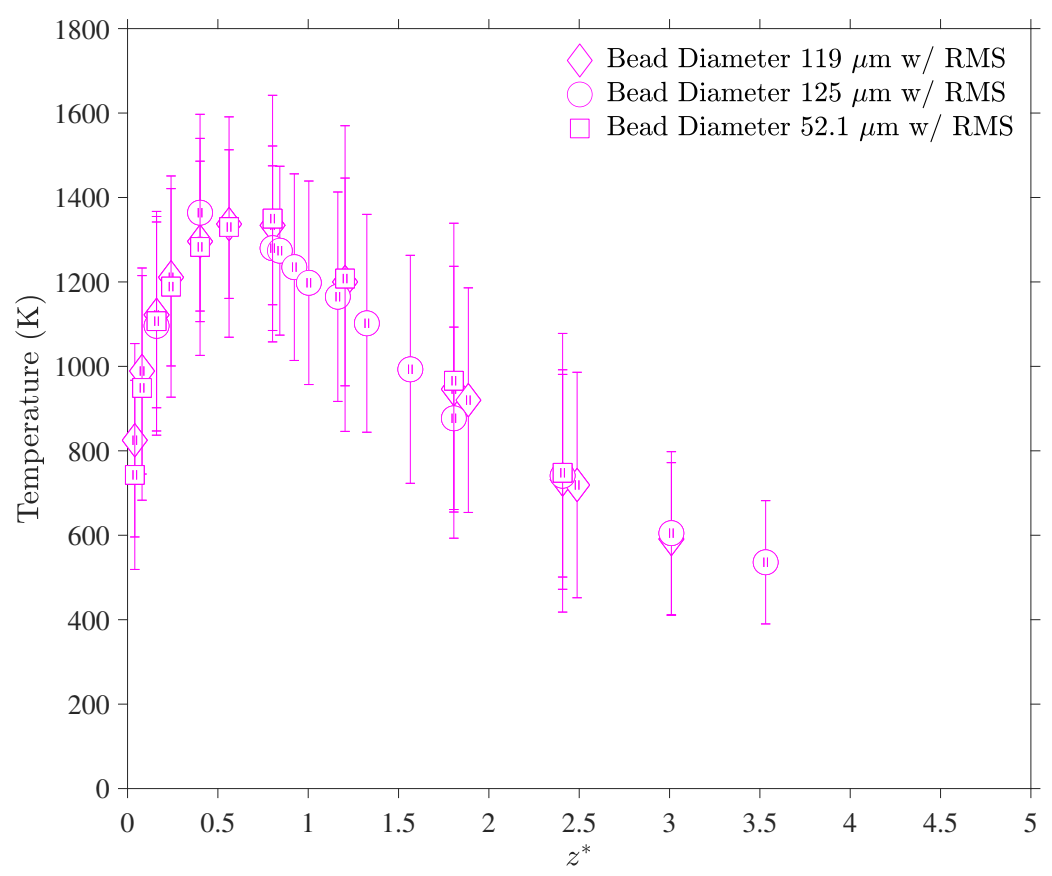

Fig. B.4. Mean and root mean square (RMS) centerline bead temperature profiles of methane as a function of $z^{*}$ 


\section{B.5 Propane (21 kW)}

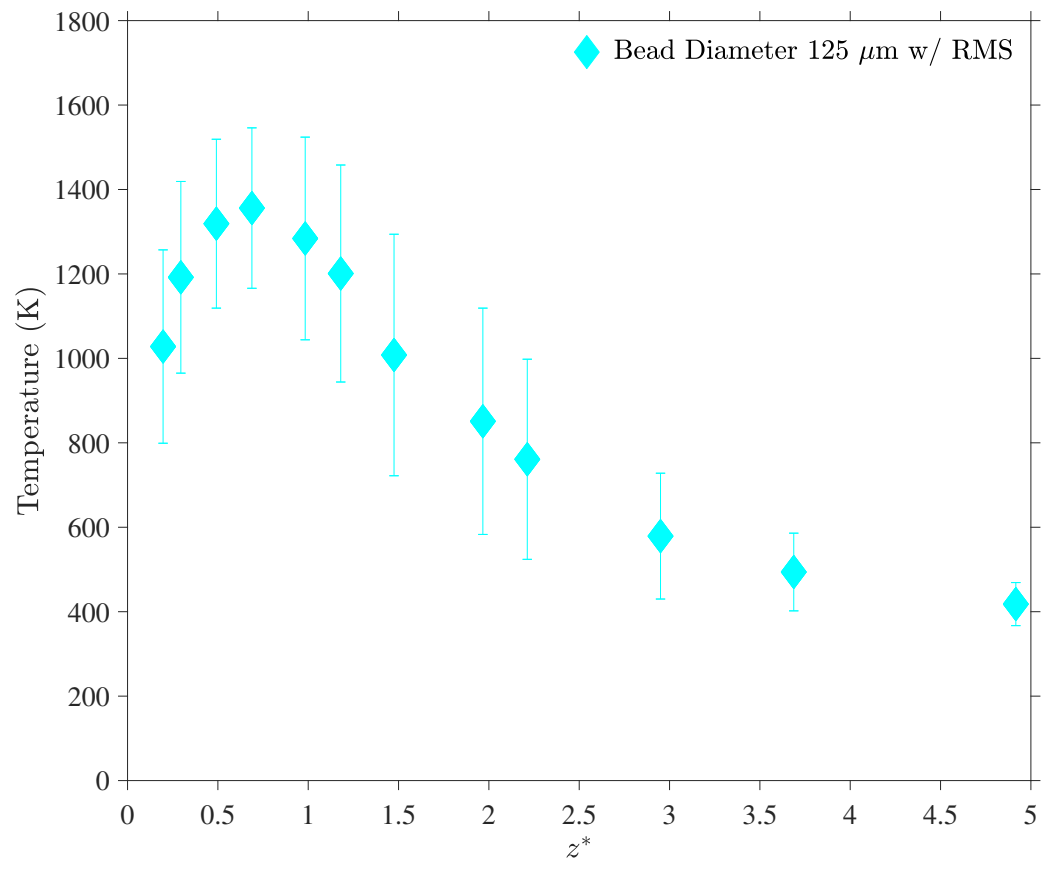

Fig. B.5. Mean and root mean square (RMS) centerline bead temperature profiles of a $21 \mathrm{~kW}$ propane fire as a function of $z^{*}$ 


\section{B.6 Propane $(34 \mathrm{~kW})$}

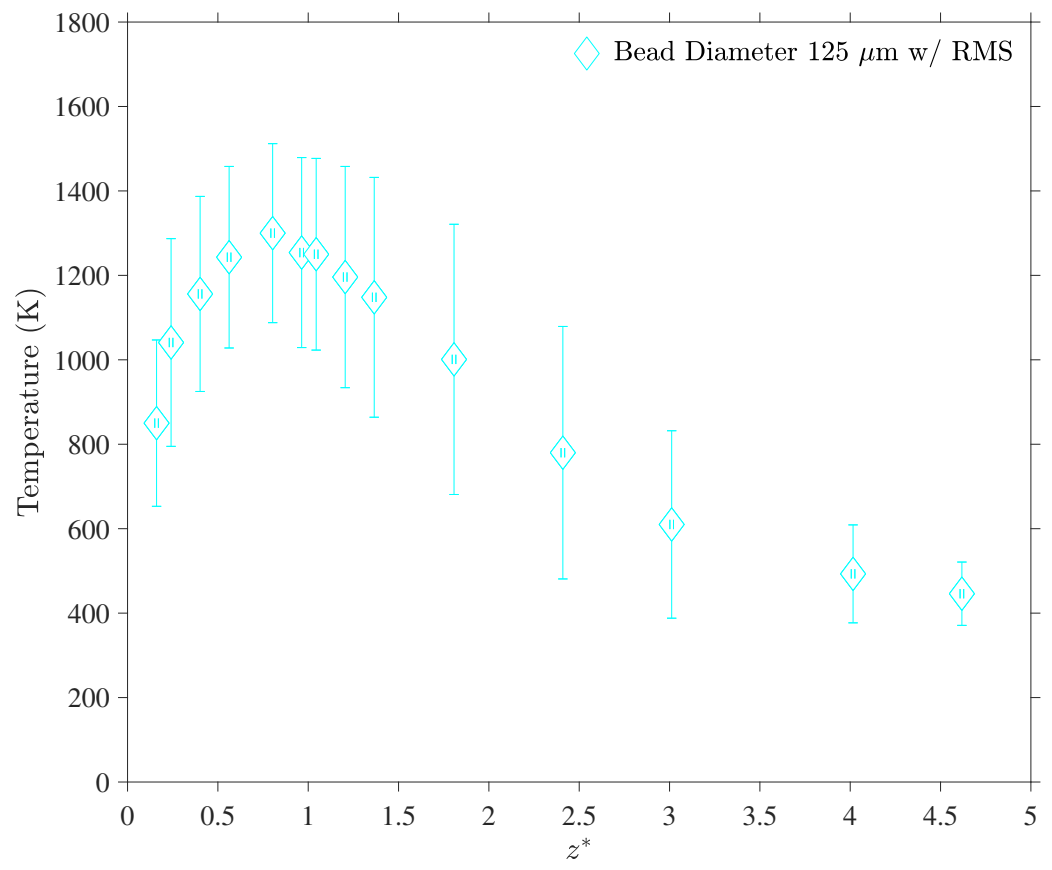

Fig. B.6. Mean and root mean square (RMS) centerline bead temperature profiles of a $34 \mathrm{~kW}$ propane fire as a function of $z^{*}$ 


\section{Estimating the Characteristic Length of the Regime of Influence}

When extracting a gas sample from a fire scene, a probe is typically placed in an arbitrary location within the fire, where it can sample gases at a preset volumetric flow rate, $\dot{V}$. Ideally, the probe inlet flow should be minimal, such that it does not disrupt the flow field created from the fire. It is assumed that, in most cases, the flow field around the inlet of the sampling probe is assumed to be vertical.

The regime of influence can be defined as the area of which the presence of the sampling probe disrupts the velocity profile. Figure C.1 shows a $2 \mathrm{D}$ vector flow field with a sink placed at the center and the regime of influence bounded by the dotted line. The characteristic length of the regime of influence, $L$, is defined as the distance from the sink which the velocity profile is approximately ( $>99 \%$ ) of dominant.

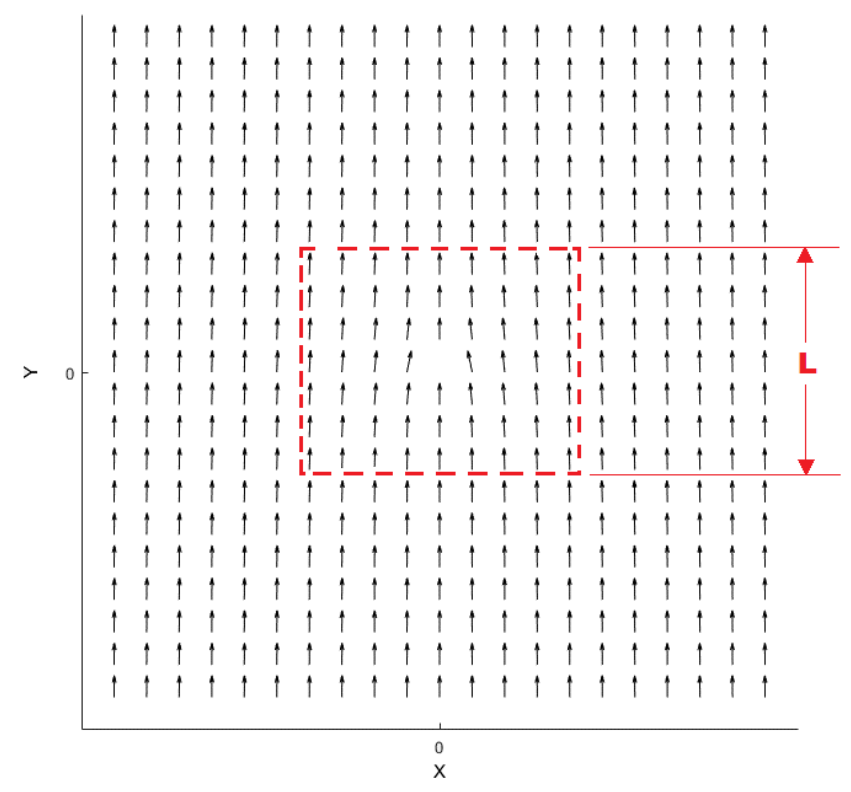

Fig. C.1. A 2D vector flow field with a sink placed at the origin. The dotted lines defines the regime of influence with a characteristic length $L$

The velocity profile, derived from potential flow is defined below:

$$
\begin{gathered}
u(x, y, z)=\frac{-\dot{V}}{8 \pi} \frac{x}{\left(x^{2}+y^{2}+z^{2}\right)^{\frac{3}{2}}} \\
v(x, y, z)=\frac{-\dot{V}}{8 \pi} \frac{y}{\left(x^{2}+y^{2}+z^{2}\right)^{\frac{3}{2}}}+V_{\infty}
\end{gathered}
$$

where $V_{\infty}$ is the velocity profile, assumed to be completely vertical surrounding the sampling probe.

$$
w(x, y, z)=\frac{-\dot{V}}{8 \pi} \frac{z}{\left(x^{2}+y^{2}+z^{2}\right)^{\frac{3}{2}}}
$$


At $(0, L, 0)$ the vertical velocity can be defined as

$$
0.99 V_{\infty}-V_{\infty}=\frac{-\dot{V}}{8 \pi} \frac{L}{L^{3}}+V_{\infty}
$$

Equation C. 4 can then be rearranged to solve for $L$.

$$
L=\sqrt{\frac{\dot{V}}{8 \pi 0.01 V_{\infty}}}
$$




\section{Elution times of combustion species using a Select for Permanent Gasses-Dual Column}

A table of the gas species elution times using the method described in Section 2.6. This method utilizes an Agilent J\&W Select Permanent Gases/ $\mathrm{CO}_{2}$ High-Resolution column is provided below.The column is a parallel configuration consisting of a $25 \mathrm{~m} \times 0.32 \mathrm{~mm}$ PoraBOND Q capillary column coupled with a $50 \mathrm{~m}$ x $0.53 \mathrm{~mm}$ Molsieve $5 \AA$ capillary column.

Elution times of gas species from a dual column composed of Molsieve 5A column and PoraBOND Q columns in parallel.

\begin{tabular}{cll}
\hline $\begin{array}{c}\text { Retention } \\
\text { Time }(\mathbf{m i n})\end{array}$ & Species & $\begin{array}{c}\text { Eluted } \\
\text { from }\end{array}$ \\
\hline 6.4 & Composite 1 & PoraBOND Q \\
6.8 & Methane & PoraBOND Q \\
7.6 & Carbon Dioxide & PoraBOND Q \\
9.2 & Composite 2 & PoraBOND Q \\
9.3 & Hydrogen & Molsieve 5 \\
12.7 & Water & PoraBOND Q \\
13.8 & Argon & Molsieve 5 \\
14.6 & Oxygen & Molsieve 5 \\
15.7 & Propane & PoraBOND Q \\
19.7 & Methanol & PoraBOND Q \\
20.6 & Nitrogen & Molsieve 5 \\
24.2 & Ethanol & PoraBOND Q \\
24.4 & Methane & Molsieve 5 \\
27.4 & Acetone & PoraBOND Q \\
29.3 & Carbon Monoxide & PoraBOND Q \\
34.7 & Benzene & PoraBOND Q \\
43.6 & Ethane & Molsieve 5 \\
50.2 & Acetylene & Molsieve 5 \\
57.3 & Ethylene & Molsieve 5 \\
65.2 & Propane & Molsieve 5 \\
\hline
\end{tabular}




\section{E. Uncertainty Analysis of Gas Species Concentrations}

\section{E.1 Uncertainty of Volume Fractions}

As shown in Eq. 9, volume fraction, $\bar{X}_{i}$, is calculated from the ratio between the number of moles of a given species, $n_{i}$, and the total number of moles identified, $n_{\text {tot. }}$ The uncertainty of the measured volume fraction is estimated using the law of propagation of uncertainty after determining the volume fraction of each species:

$$
u_{\bar{X}_{\mathrm{i}}}=\sqrt{\left(\frac{\partial \bar{X}_{i}}{\partial n_{i}} u_{n_{i}}\right)^{2}+\left(\frac{\partial \bar{X}_{i}}{\partial n_{\mathrm{tot}}} u_{n_{\mathrm{tot}}}\right)^{2}}
$$

A coverage factor of 2 is applied to the combined uncertainty to produce a $95 \%$ confidence interval.

\section{E.1.1 Number of Moles of a Given Species}

The number of moles of a given species is determined from a calibration function of the integrated peak area of the respective species obtained from the TCD's and the MS's Total Ion Current (TIC) chromatograms. The Type A evaluation of standard uncertainty of the number of moles of a given species is taken as the standard deviation of the measurements obtained from the repeated experiments. The Type B evaluation of standard uncertainty is determined from the error in the calibration functions for each species measured by the TCD and MS is further detailed in Appendix F. The combined uncertainty is found via quadrature:

$$
u_{n_{i}}=\sqrt{u_{n_{i, \mathrm{cal}}}^{2}+s_{n_{i}}^{2}}
$$

\section{E.1.2 Total Number of Moles Identified}

The total number of moles detected is determined from the summation of the number of moles for each species identified by the TCD and TIC chromatograms. Therefore, the uncertainty in the total number of moles identified is the combined uncertainty of all the identified species via quadrature:

$$
u_{n_{\mathrm{tot}}}=\sqrt{\sum_{n=1}^{N} u_{n_{i}}^{2}}
$$

where $N$ is the number of a species identified species in the TCD and TIC chromatogram.

\section{E.2 Uncertainty of Mass Fractions}

Mass fraction of a given species is calculated using its measured volume fraction, $\bar{X}_{i}$, molecular weight, $W_{i}$, and the average molecular weight of all detected gas species, $W_{\text {tot }}$, as 
shown in Eq. 10. The uncertainty of the mass fraction of a given species is estimated from the law of propagation of uncertainty using Eq. 10.

$$
u_{\bar{Y}_{\mathrm{i}}}=\sqrt{\left(\frac{\partial \bar{Y}_{i}}{\partial \bar{X}_{i}} u_{\bar{X}_{i}}\right)^{2}+\left(\frac{\partial \bar{Y}_{i}}{\partial W_{\mathrm{tot}}} u_{W_{\mathrm{tot}}}\right)^{2}}
$$

\section{E.2.1 Uncertainty of the average molecular weight}

The uncertainty of the average molecular weight is determined using the law of propagation of uncertainty, which accounted for each detected species measured from the injected sample.

$$
u_{W_{\mathrm{tot}}}=\sqrt{\sum\left(u_{\bar{X}_{i}} W_{i}\right)^{2}}
$$




\section{F. Uncertainty Analysis of Gas Species Calibrations}

Peak areas are converted into number of moles, $n_{i}$, using linear calibration curves.

$$
n_{i}=a(\text { Area })+\mathrm{b}
$$

The coefficients in the calibration curves are weighted to account for the error of each gas standard used in the calibration procedure. The uncertainty of a number of moles determined from calibration function is estimated using the law of propagation of uncertainty:

$$
u_{\mathrm{n}_{\mathrm{i}}}=\sqrt{\left(\frac{\partial n_{i}}{\partial a} u_{a}\right)^{2}+\left(\frac{\partial n_{i}}{\partial b} u_{b}\right)^{2}}
$$

The uncertainties of the slope and intercept in a weighting linear regression are as follows:

$$
\begin{aligned}
& u_{\mathrm{a}}=\sqrt{\frac{\sum \frac{1}{u_{n_{i, \mathrm{cal}}}}}{\left(\sum \frac{\text { Area }_{\mathrm{i}}^{2}}{u_{n_{i, \mathrm{cal}}}^{2}}\right)\left(\sum \frac{1}{u_{n_{i, \mathrm{cal}}}^{2}}\right)-\left(\sum \frac{\text { Area }_{\mathrm{i}}}{u_{n_{i, \mathrm{cal}}}^{2}}\right)^{2}}} \\
& u_{\mathrm{b}}=\sqrt{\frac{\sum \frac{\text { Area }_{\mathrm{i}}^{2}}{u_{n_{i, \mathrm{cal}}}}}{\left(\sum \frac{\text { Area }_{\mathrm{i}}^{2}}{u_{n_{i, \mathrm{cal}}}^{2}}\right)\left(\sum \frac{1}{u_{n_{i, \mathrm{cal}}}^{2}}\right)-\left(\sum \frac{\text { Area }_{\mathrm{i}}}{u_{n_{i, \mathrm{cal}}}^{2}}\right)^{2}}}
\end{aligned}
$$

where $u_{n_{i, \text { cal }}}$ is the uncertainty of a known number of moles injected into the GC/MS for calibration.

During calibration, the number of moles of a given species $n_{i, \mathrm{cal}}$ are calculated from the product of the total moles injected into the GC/MS, $n_{\text {inj }}$, and the known concentration of the particular species in the calibration standard, $C_{i}$.

$$
n_{i, \mathrm{cal}}=C_{i} n_{\mathrm{inj}}
$$

A collection of gas calibration standards for a variety of species are pre-selected to provide a broad range of concentrations. All calibration standards are mixtures of the target gas species with a nitrogen balance, with the exception of one standard balanced in Air. A list of gas standards used in this work, with their respective concentrations and Type B evaluation of standard uncertainty, is provided in Appendix F.2.

The uncertainty of the number of moles of a given species injected into the GC/MS for calibration is estimated using the law of propagation of uncertainty:

$$
u_{\mathrm{n}_{\mathrm{i}, \mathrm{cal}}}=\sqrt{\left(\frac{\partial n_{i, \mathrm{cal}}}{\partial C_{i}} u_{C_{i}}\right)^{2}+\left(\frac{\partial n_{i, \mathrm{cal}}}{\partial n_{\mathrm{inj}}} u_{n_{\mathrm{inj}}}\right)^{2}}
$$




\section{F.1 Total Moles Injected into the GC/MS for Calibation}

The total moles injected into the GC/MS, $n_{\mathrm{inj}}$, for calibration is determined from Eq. (16) using the pressure, $P$, temperature, $T$, and volume, $V_{\mathrm{s}}$, of the gas sample injected into the GC/MS. Pressure and temperature measurements are made using a digital pressure gauge (OMEGA P10R-001) and K-type thermocouple located at the GC/MS sample loop injection valve, respectively, sampling at $2 \mathrm{~Hz}$ for $50 \mathrm{~s}$. The volume of the GC/MS sample loop is $2 \mathrm{~mL}$. The Type A evaluation of uncertainty of the total moles injected into the GC/MS for calibration is determined from the standard error of the pressure, $s_{P}$, and temperature, $s_{T}$ readings from the sampling period. The Type B evaluation of uncertainty for the total moles injected into the GC/MS for calibration is determined from the bias error sources in the instrumentation, $u_{\mathrm{inst}}$, used to measure pressure $(0.008 \%$ accuracy of the reading) and temperature $\left(1.5^{\circ} \mathrm{C}\right)$ of gas sample injected. The combined uncertainty of the temperature measurements is found via quadrature:

$$
u_{T}=\sqrt{u_{\text {inst }}^{2}+s_{T}^{2}}
$$

The Type A uncertainty is the dominant contribution for the uncertainty of the pressure measurements, therefore its uncertainty of pressure is approximately the standard deviation.

$$
u_{P} \approx s_{P}^{2}
$$

The standard uncertainty of the total moles injected into the GC/MS for calibration is estimated using the law of propagation of uncertainty:

$$
u_{n_{\mathrm{inj}}}=\sqrt{\left(\frac{\partial n_{\mathrm{inj}}}{\partial P} u_{P}\right)^{2}+\left(\frac{\partial n_{\mathrm{inj}}}{\partial T} u_{T}\right)^{2}}
$$




\section{F.2 Table of Gas Standards with Error}

A table of the gas standards with their respective concentrations and Type B evaluation of standard uncertainty, used for calibrating the GC/MS is provided below. Lot numbers for all standards are provided for traceability.

Table F.1. Gas standards used to calibrate the GC/MS

\begin{tabular}{|c|c|c|c|}
\hline Components & Uncertainty(\%) & Distributor & Lot No. \\
\hline 200 ppm Acetone & 2.00 & Gasco Affiliates, LLC. & DNJ-ACE-200N-1 \\
\hline $0.26 \%$ Acetylene & 2.00 & Gasco Affiliates, LLC. & FBJ-M24-0.25\%-1 \\
\hline 1.04\% Acetylene & 2.00 & Gasco Affiliates, LLC. & FBJ-M24-1 \\
\hline $1.02 \%$ Argon & 2.00 & Gasco Affiliates, LLC. & DBJ-2-1N-1 \\
\hline $88.5 \%$ Argon & 2.00 & Gasco Affiliates, LLC. & DBJ-2-90N-1 \\
\hline 100 ppm Benzene & 2.00 & Gasco Affiliates, LLC. & FBJ-21-100-3 \\
\hline 15.6\% Carbon Dioxide & 0.04 & NIST Gas Sensing Metrology Group & $9-\mathrm{C}-44$ \\
\hline 24.5\% Carbon Dioxide & 2.00 & Gasco Affiliates, LLC. & KBI-35-25-1 \\
\hline 1.00\% Carbon Dioxide & 2.00 & Matheson Tri-Gas & 9306620888 \\
\hline 2.51\% Carbon Dioxide & 2.00 & Roberts Oxygen & 1002080917 \\
\hline 7.00\% Carbon Dioxide & 2.00 & Roberts Oxygen & 1009010318 \\
\hline 9.00\% Carbon Dioxide & 2.00 & Praxair Doistribution Inc. & 304113044702 \\
\hline $0.30 \%$ Carbon Monoxide & 2.00 & Roberts Oxygen & 1009010318 \\
\hline 0.02\% Carbon Monoxide & 2.00 & Matheson Tri-Gas & 9306620888 \\
\hline 0.11\% Carbon Monoxide & 2.00 & Roberts Oxygen & 1002080917 \\
\hline 4.00\% Carbon Monoxide & 2.00 & Praxair Doistribution Inc. & 304113044702 \\
\hline 7.81\% Carbon Monoxide & 0.02 & NIST Gas Sensing Metrology Group & $51-28-\mathrm{C}$ \\
\hline $0.51 \%$ Ethane & 2.00 & Gasco Affiliates, LLC. & FBJ-62N-0.5-1 \\
\hline $1.00 \%$ Ethane & 2.00 & Gasco Affiliates, LLC. & FBJ-152N-1-1\%-1 \\
\hline $2.55 \%$ Ethane & 2.00 & Gasco Affiliates, LLC. & FBJ-152N-2.5-1 \\
\hline $0.51 \%$ Ethylene & 2.00 & Gasco Affiliates, LLC. & FBJ-62N-0.5\%-1 \\
\hline $1.02 \%$ Ethylene & 2.00 & Gasco Affiliates, LLC. & FBJ-62N-1\%-1 \\
\hline $2.55 \%$ Ethylene & 2.00 & Gasco Affiliates, LLC. & FBJ-62N-2.5\%-1 \\
\hline $0.26 \%$ Hydrogen & 2.00 & Gasco Affiliates, LLC. & FBJ-84-0.25-1 \\
\hline $0.50 \%$ Hydrogen & 2.00 & Gasco Affiliates, LLC. & KBI-84-0.5-1 \\
\hline $1.00 \%$ Hydrogen & 2.00 & Gasco Affiliates, LLC. & KBI-84-1-3 \\
\hline 2.00\% Hydrogen & 2.00 & Gasco Affiliates, LLC. & FBJ-84-2-5 \\
\hline 4.03\% Hydrogen & 2.00 & Gasco Affiliates, LLC. & FBJ-84-4-2 \\
\hline $0.40 \%$ Methane & 2.00 & Gasco Affiliates, LLC. & DBJ-135N-0.4-1 \\
\hline 3.95\% Methane & 2.00 & Gasco Affiliates, LLC. & DBJ-135N-4-2 \\
\hline $40.8 \%$ Methane & 2.00 & Gasco Affiliates, LLC. & DBJ-135N-40-1 \\
\hline $0.50 \%$ Oxygen & 2.00 & Gasco Affiliates, LLC. & DBJ-2-90N-1 \\
\hline $1.97 \%$ Oxygen & 0.01 & NIST Gas Sensing Metrology Group & 73-D-03 \\
\hline $5.02 \%$ Oxygen & 2.00 & Gasco Affiliates, LLC. & DBJ-161-5-5 \\
\hline $9.92 \%$ Oxygen & 0.02 & NIST Gas Sensing Metrology Group & 72-D-60 \\
\hline $10.2 \%$ Oxygen & 2.00 & Gasco Affiliates, LLC. & KBI-161-10-6 \\
\hline $20.7 \%$ Oxygen & 0.04 & NIST Gas Sensing Metrology Group & 71-D-51 \\
\hline $0.42 \%$ Propane & 2.00 & Gasco Affiliates, LLC. & DBJ-176N-0.4-1 \\
\hline $39.6 \%$ Propane & 2.00 & Gasco Affiliates, LLC. & DBJ-176N-40-1 \\
\hline
\end{tabular}




\section{F.3 Concentration of Vapors from Bubblers}

The volume fraction of vapor from liquid materials are calibrated from the ratio of the liquid-vapor pressure in the heated flask to the total pressure in the flask.

$$
C_{\mathrm{vap}}=\frac{P_{\mathrm{vap}}}{P}
$$

The bubbler setup shown in Fig. F.1:

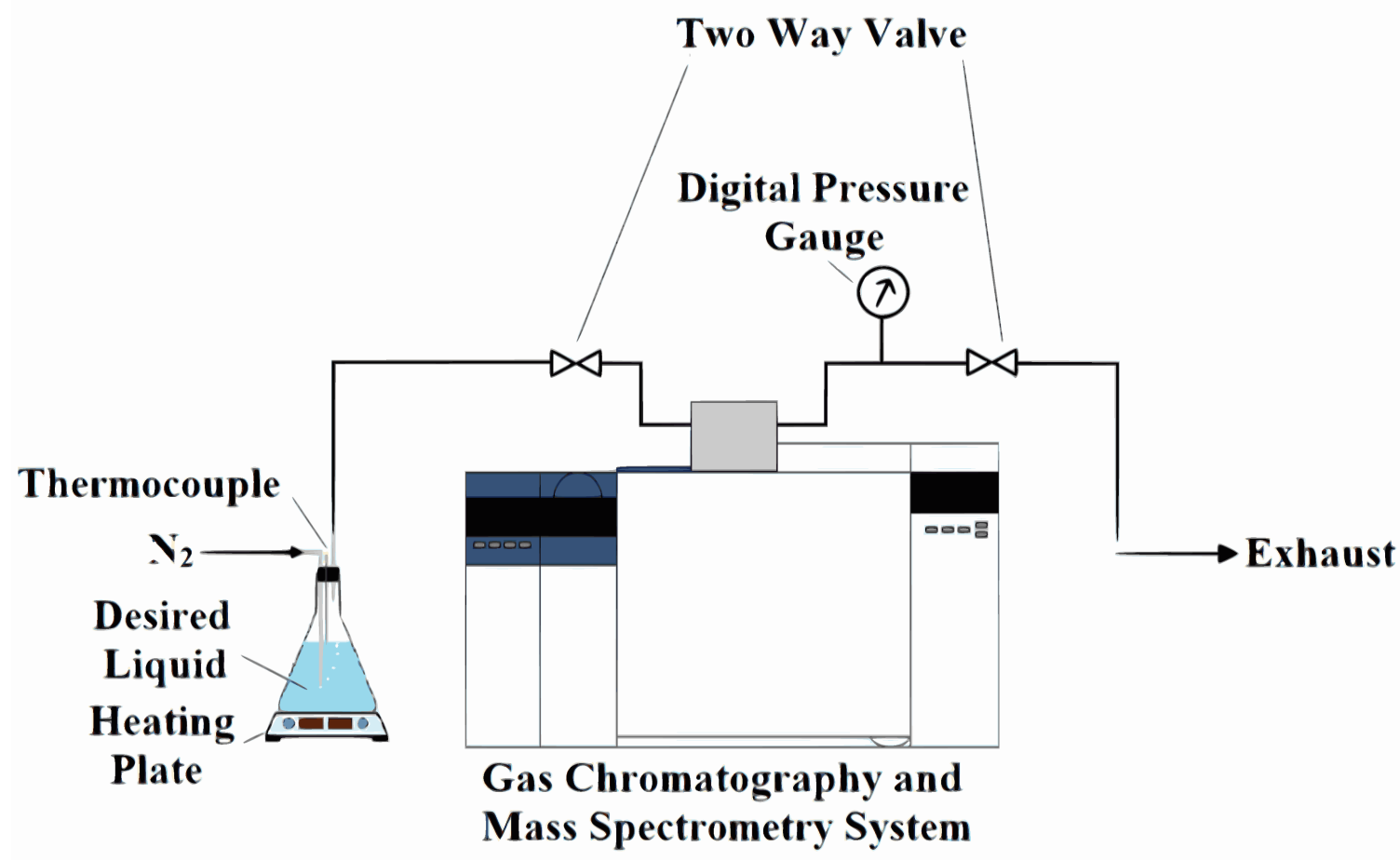

Fig. F.1. Flow diagram for bubble calibration system used for liquid materials (acetone, ethanol, methanol, and water)

The material of interest is placed in a $500 \mathrm{~mL}$ Pyrex flask sitting on a heating plate. Nitrogen, acting as a carrier gas, is bubbled through the liquid bath and then transported through a heated gas line and into the GC/MS sample loop. Vapor from the liquid is transported along with the carrier gas in an amount liquid depending on the temperature. The concentration of the vapor injected into the GC/MS is calculated from a liquid-vapor pressure correlation provided by DIPPR [9].

$$
P_{\text {vap }}=\mathrm{e}^{A+\frac{B}{T_{\mathrm{B}}}+C \ln \left(T_{\mathrm{B}}\right)+D\left(T_{\mathrm{B}}\right)^{E}}
$$

In this correlation, $P_{\text {vap }}$ is the vapor pressure calculated from the temperature of the liquid bath, $T_{\mathrm{B}}$, with the coefficients $(A, B, C, D, E)$ specific to the liquid material. Table F.2 lists all coefficients for each calibrated liquid, including the uncertainty of their respective correlations, $u_{\text {corr }}$. 
Table F.2. Liquid vapor pressure correlation coefficients for various calibrated liquids

\begin{tabular}{lcccccc}
\hline Liquid Material & A & B & C & D & E & Uncertainty(\%) \\
\hline & & & & & & \\
Acetone & 69.006 & -5599.6 & -7.0985 & $6.2237 \mathrm{E}-6$ & 2.00 & 3.00 \\
Ethanol & 73.304 & -7122.3 & -7.1424 & $2.8853 \mathrm{E}-6$ & 2.00 & 1.00 \\
Methanol & 82.718 & -6904.5 & -8.8622 & $7.4664 \mathrm{E}-6$ & 2.00 & 3.00 \\
Water & 73.649 & -7258.2 & -7.3037 & $4.1653 \mathrm{E}-6$ & 2.00 & 0.20 \\
\hline
\end{tabular}

The concentration range of each calibrated liquid is approximately $2 \%$ to $50 \%$. Liquid bath temperatures are controlled using a heating plate positioned underneath the insulated bubbler. The temperature of the bath is measured using a K-type thermocouple placed at the liquid surface. The bath temperature measurements are sampled at $2 \mathrm{~Hz}$ for $50 \mathrm{~s}$ simultaneously with pressure and temperature measurements of the GC/MS sample loop. Liquid-vapor calibrations are conducted once the bath reaches a steady-state temperature (approximately $1 \mathrm{~h}$ ) and the nitrogen/vapor gas mixture has swept through the sample loop. Upon injection into the GC/MS, pressure and temperature measurements of the sample loop are made as described in Appendix F.1.

The uncertainty of the concentration determined using Eq. (F.10) is estimated using the law of propagation of uncertainty:

$$
u_{C_{\mathrm{vap}}}=\sqrt{\left(\frac{\partial C_{\mathrm{vap}}}{\partial P} u_{P}\right)^{2}+\left(\frac{\partial C_{\mathrm{vap}}}{\partial P_{\mathrm{vap}}} u_{P_{\mathrm{vap}}}\right)^{2}}
$$

The uncertainty of the pressure measured upon injected is calculated from Eq. (F.8). The uncertainty of the vapor pressure is found by combining the propagated error of liquid bath temperature and the uncertainty in the correlation via quadrature:

$$
u_{P_{\mathrm{vap}}}=\sqrt{\left(\frac{\partial P_{\mathrm{vap}}}{\partial T_{\mathrm{B}}} u_{T_{\mathrm{B}}}\right)^{2}+u_{\mathrm{corr}}^{2}}
$$

The Type A evaluation of uncertainty of the liquid bath temperature readings is determined from the standard error of the temperature, $s_{T_{\mathrm{B}}}$ readings from the sampling period. The Type B evaluation of uncertainty for the liquid bath temperature is defined as the bias error source $\left(1.5^{\circ} \mathrm{C}\right)$ in the thermocouple, $u_{\text {inst }}$. The combined uncertainty liquid bath temperature is determined via quadrature:

$$
u_{T_{\mathrm{B}}}=\sqrt{u_{\mathrm{inst}}^{2}+s_{T_{\mathrm{B}}}^{2}}
$$




\section{G. Uncertainty Analysis of the Soot Mass Fraction}

The local soot mass fraction measurements, $Y_{\mathrm{s}}$, made at various heights above the fuel surface are calculated through a combination of Eqs. (11), (12), and (13):

$$
Y_{\mathrm{s}}=\frac{m_{\mathrm{s}} V_{s}}{\dot{V} \Delta t m_{\mathrm{det}}} \frac{T_{\infty}}{T_{\mathrm{g}}}
$$

where $m_{\mathrm{s}}$ is the mass of soot collected on the PTFE filter and gun cleaning patches, $\dot{V}$ is the volumetric flow rate, $V_{s}$ is the volume of the sample loop, $m_{\mathrm{det}}$ is the total mass of the gas sample detected in the TCD and TIC chromatograms, $T_{\infty} / T_{\mathrm{g}}$ is the ratio of the internal gas flow temperature readings of the mass flow controller to the temperature of the probe, and $\Delta t$ is the total sampling time. The uncertainty of the measured soot mass fraction is estimated using the law of propagation of uncertainty after determining the soot mass fraction:

$$
u_{Y_{\mathrm{s}}}=\sqrt{\left(\frac{\partial Y_{\mathrm{s}}}{\partial m_{\mathrm{s}}} u_{m_{\mathrm{s}}}\right)^{2}+\left(\frac{\partial Y_{\mathrm{s}}}{\partial \dot{V}} u_{\dot{V}}\right)^{2}+\left(\frac{\partial Y_{\mathrm{s}}}{\partial m_{\mathrm{det}}} u_{m_{\mathrm{det}}}\right)^{2}}
$$

The uncertainty of the temperature measurements is not accounted for since the uncertainty of the soot mass fraction is dominated by the remaining parameters uncertainties.

\section{G.1 Mass of Soot}

The mass of soot is determined from the difference in mass of the dried PTFE filter and gun cleaning patches before and $48 \mathrm{~h}$ after each experiment. The Type A evaluation of standard uncertainty of the mass of soot, $m_{\mathrm{s}}$, is taken as the standard deviation, $s_{m_{\mathrm{s}}}$, of the measurements sampled three times before and after each experiment. The Type B evaluation of uncertainty, $u_{\text {inst }}$, is determined from the instrumentation error sources of the scale and is found to be $1 \%$ of the reading. The Type A evaluation of uncertainty dominates; thus, the standard uncertainty is approximately the standard deviation of the multiple measurements:

$$
u_{m_{\mathrm{s}}} \approx s_{m_{\mathrm{s}}}
$$

\section{G.2 Mass Flow Controller Volumetric Flow Rate}

A mass flow controller is used to measure the volumetric flow rate, $\dot{V}$, within the gas sampling line. The Type A uncertainty is taken as the standard deviation of the flow measurements sampled at $2 \mathrm{~Hz}$ during the sampling period which varies from $12 \mathrm{~min}$ to $25 \mathrm{~min}$ depending on the sampling location within the fire. The Type B sources of uncertainty consist of the calibration error, $u_{\text {cal }}$, and the precision error sources at calibration conditions, $u_{\text {prec}}$. The calibration error is given as $2 \mathrm{~mL} / \mathrm{min}$. The precision error is $0.8 \%$ of the reading plus $0.2 \%$ of the full scale $(2 \mathrm{~L} / \mathrm{min})$. The combined uncertainty is calculated via quadrature:

$$
u_{\dot{V}}=\sqrt{u_{\mathrm{prec}}^{2}+u_{\mathrm{cal}}^{2}+s_{\dot{V}}^{2}}
$$




\section{G.3 Total Mass Detected}

The total mass detected in the TCD and TIC chromatograms, $m_{\text {det }}$, is calculated:

$$
m_{\mathrm{det}}=\sum_{n=1}^{N} n_{i} \mathrm{~W}_{i}
$$

where $n_{i}$ is the moles of species $i$ and $\mathrm{W}_{i}$ is the molar mass. The uncertainty in the total mass is calculated from the uncertainties of all identified species, defined in Section E.1.2, multiplied by their corresponding molar mass via quadrature:

$$
u_{m_{\mathrm{det}}}=\sqrt{\sum_{n=1}^{N}\left(u_{n_{i}} \mathrm{~W}_{i}\right)^{2}}
$$

where $N$ is the number of a species identified species in the chromatograms. 


\section{H. Figures of Averaged Volume Fractions}

\section{H.1 Methanol}
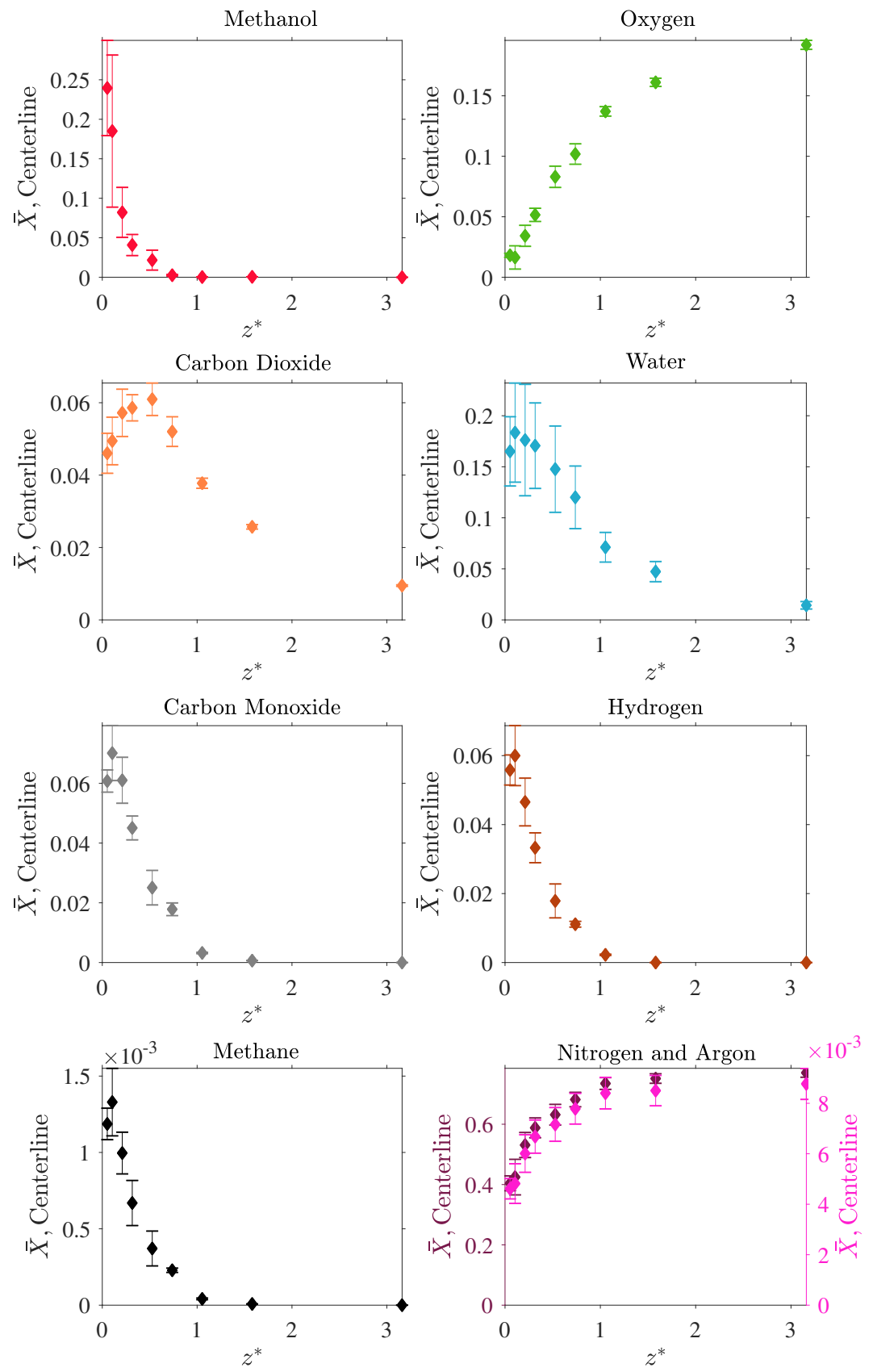

Experimental Data with Error

Fig. H.1. Plot of volume fractions of all species identified in the methanol pool fire as a function of $z^{*}$ along the pool centerline. The error is a combined uncertainty, further described in Section E. 


\section{H.2 Ethanol}
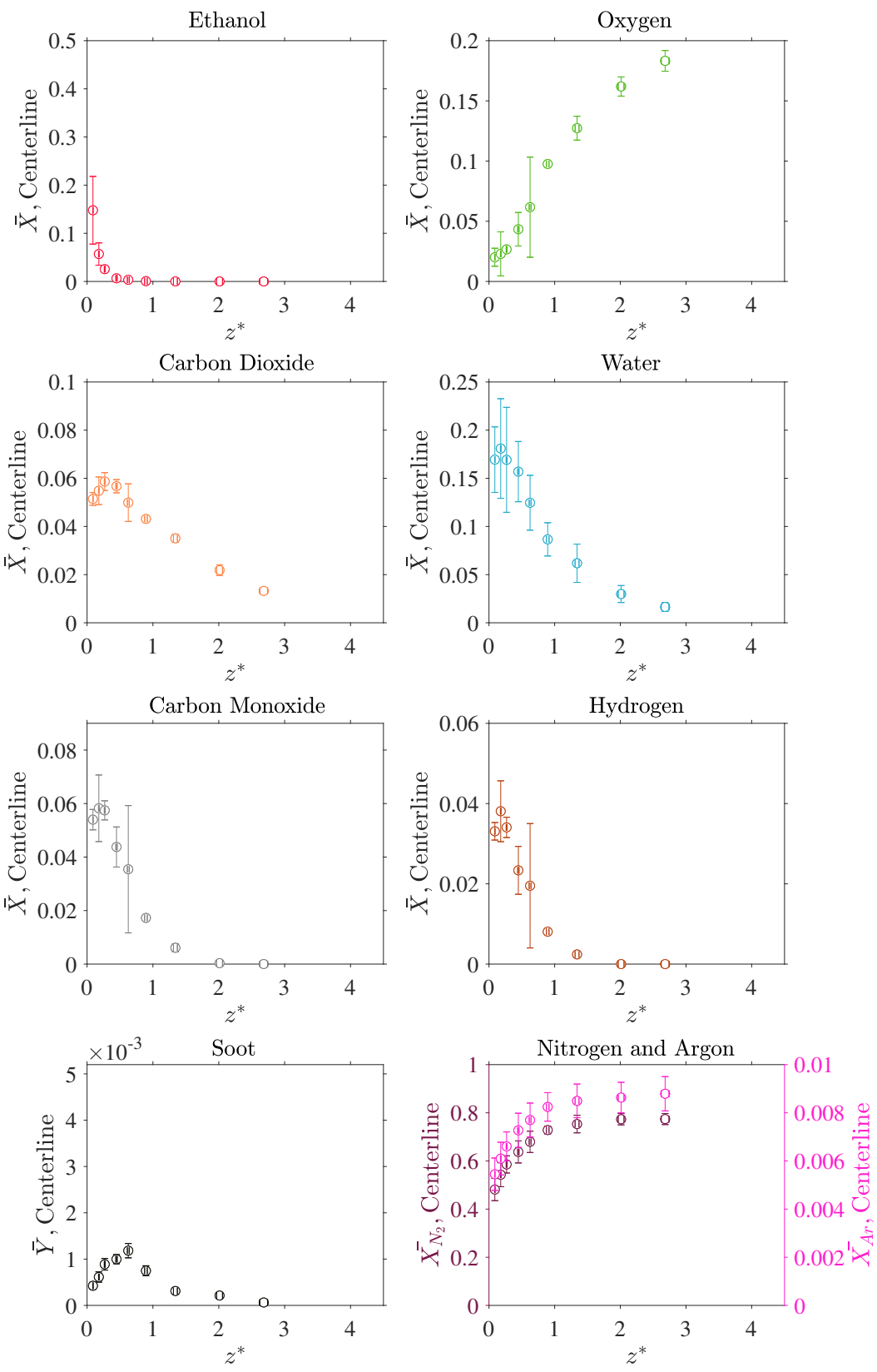

Experimental Data with Error

Fig. H.2. Plot of volume fractions of major species identified in the ethanol pool fire as a function of $z^{*}$ along the pool centerline. The error is a combined uncertainty, further described in Section E. 

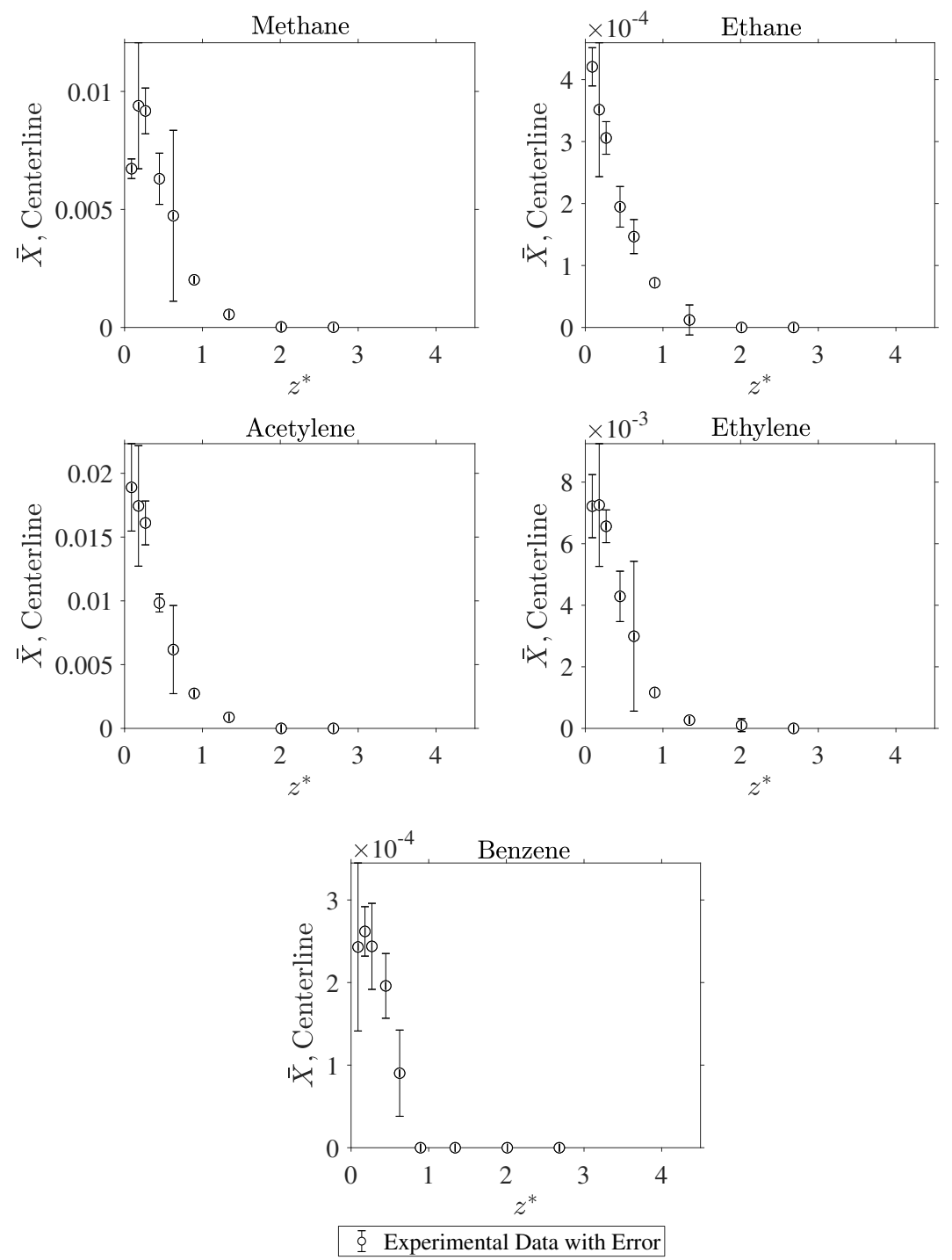

Fig. H.3. Plot of volume fractions of minor and trace species identified in the ethanol pool fire as a function of $z^{*}$ along the pool centerline. The error is a combined uncertainty, further described in Section E. 


\section{H.3 Acetone}
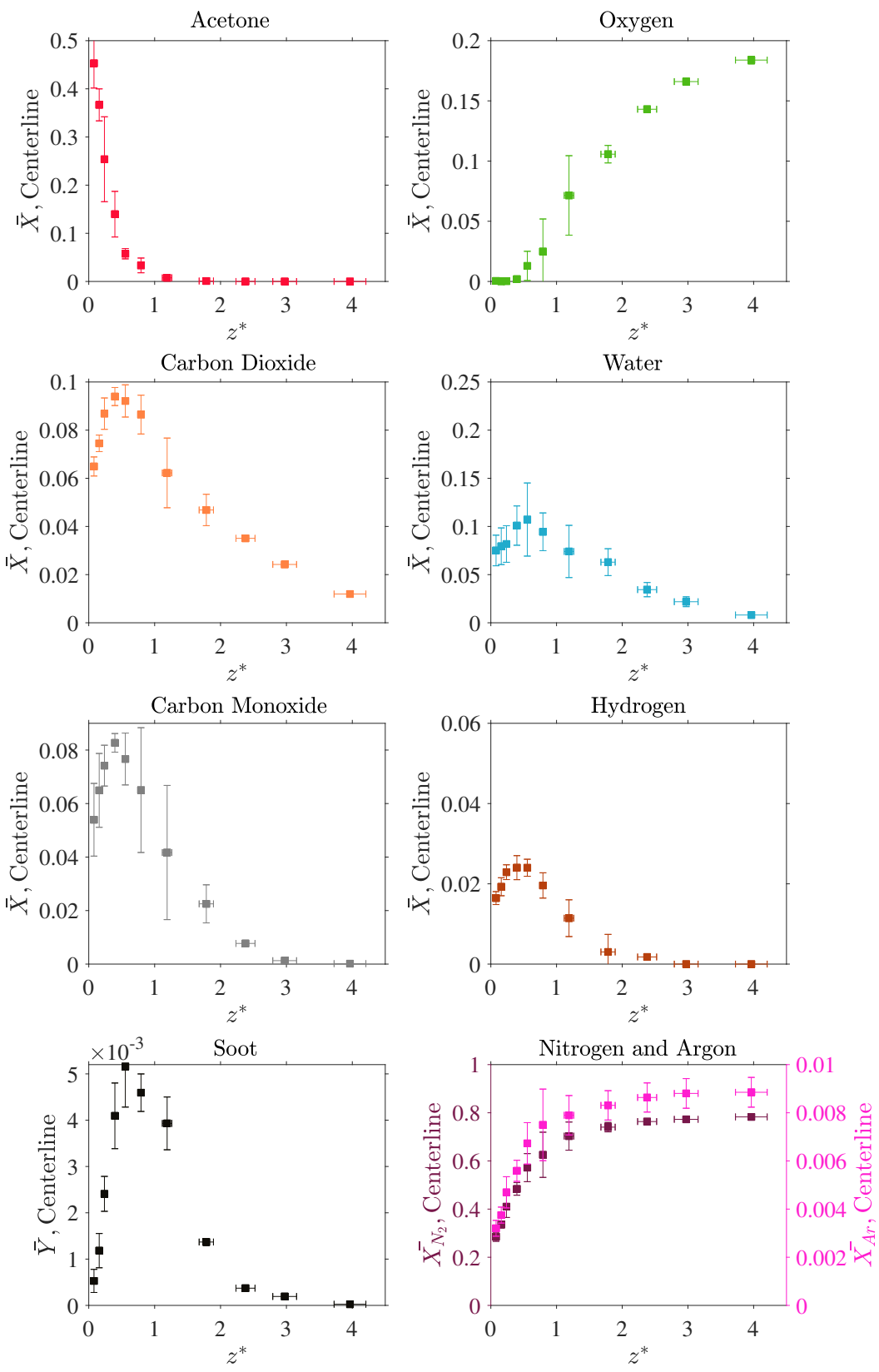

I Experimental Data with Error

Fig. H.4. Plot of volume fractions of major species identified in the acetone pool fire as a function of $z^{*}$ along the pool centerline. The error is a combined uncertainty, further described in Section E. 

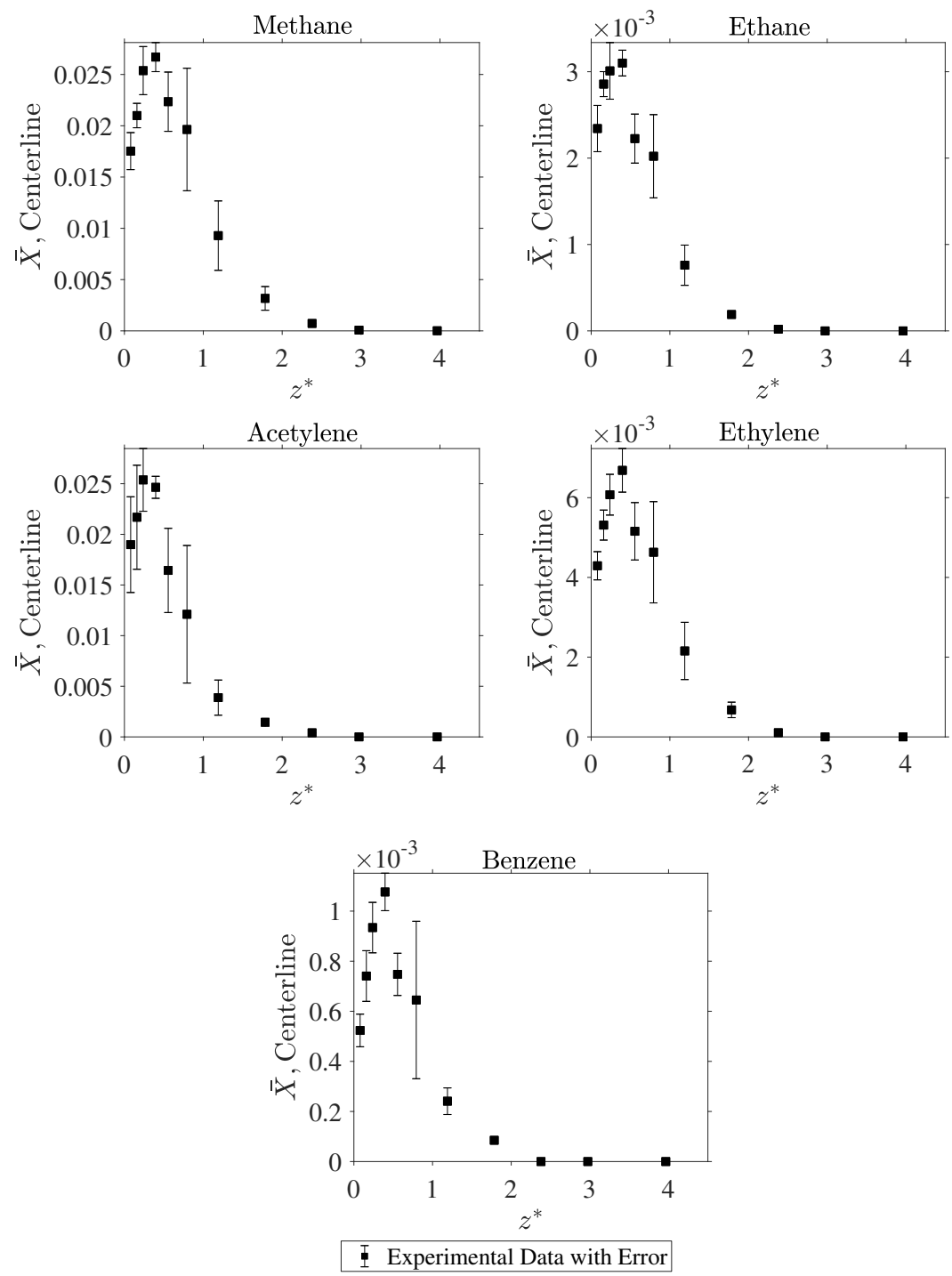

Fig. H.5. Plot of volume fractions of minor and trace species identified in the acetone pool fire as a function of $z^{*}$ along the pool centerline. The error is a combined uncertainty, further described in Section E. 


\section{H.4 Methane}
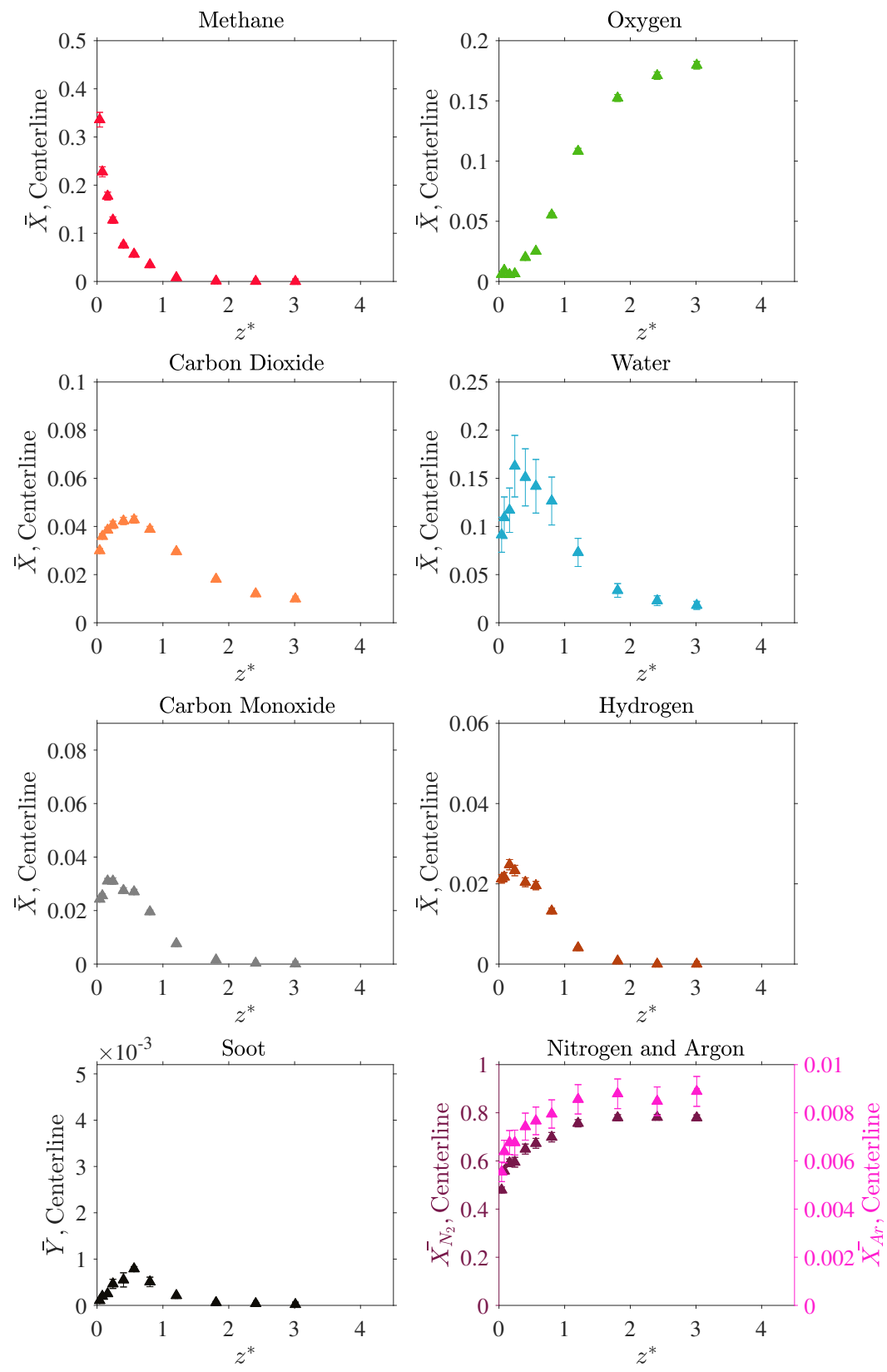

I Experimental Data with Error

Fig. H.6. Plot of volume fractions of all species identified in the methane pool fire as a function of $z^{*}$ along the pool centerline. The error is a combined uncertainty, further described in Section E. 

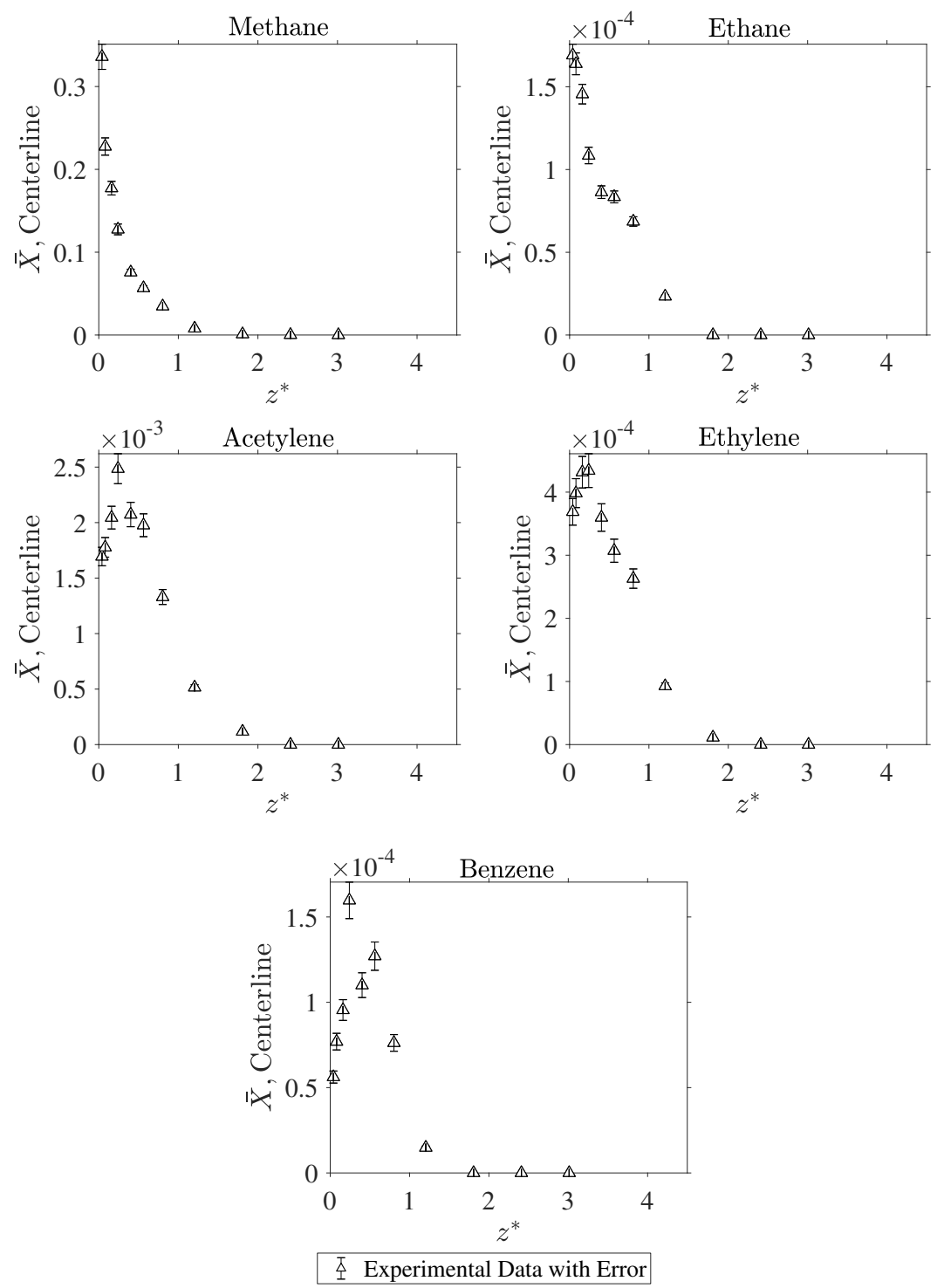

Fig. H.7. Plot of volume fractions of minor and trace species identified in the methane pool fire as a function of $z^{*}$ along the pool centerline. The error presented here is a combined uncertainty, further described in Section E. 


\section{H.5 Propane (21 kW)}
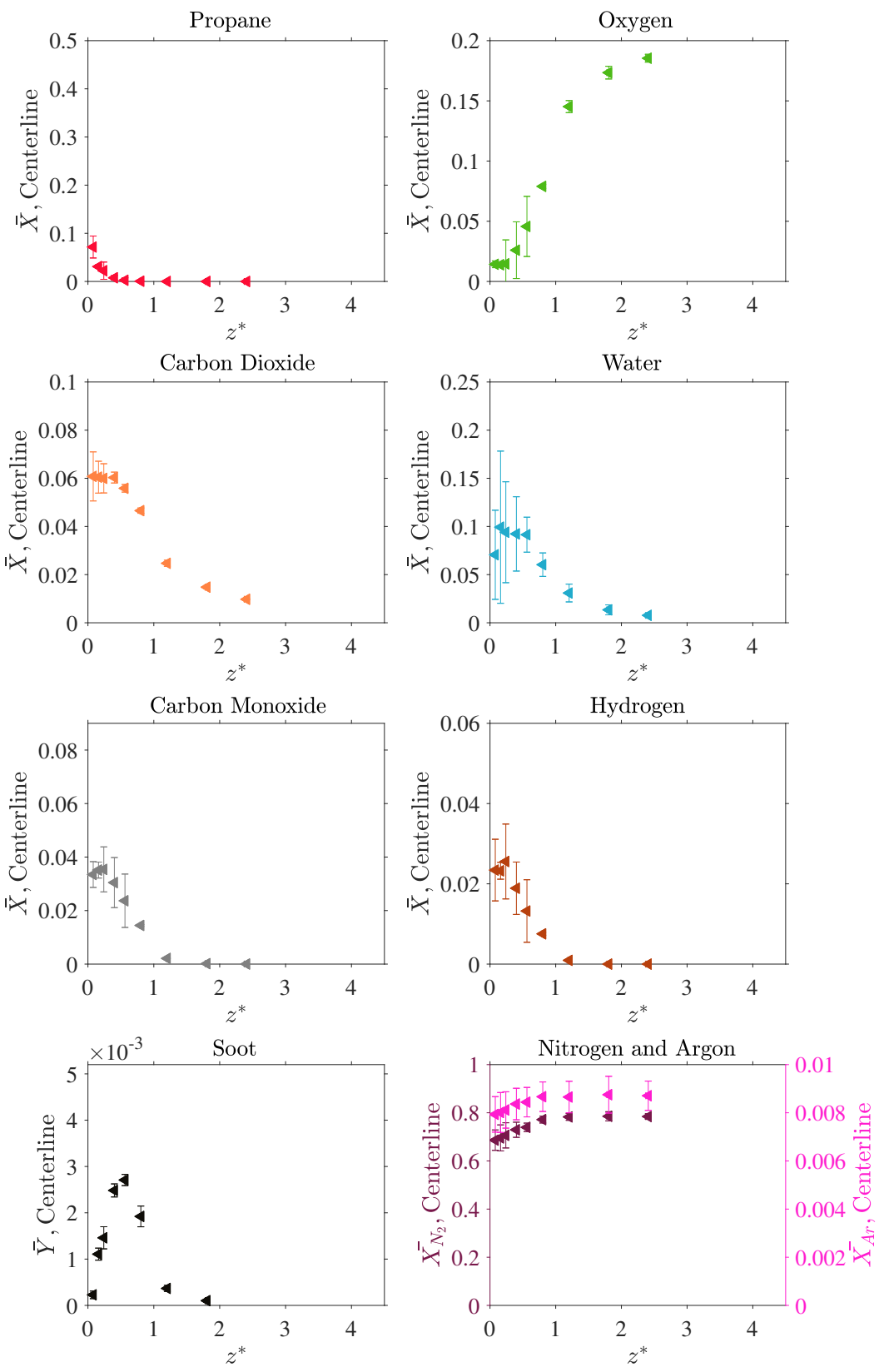

Experimental Data with Error

Fig. H.8. Plot of volume fractions of all species identified in the propane $(21 \mathrm{~kW})$ pool fire as a function of $z^{*}$ along the pool centerline. The error is a combined uncertainty, further described in Section E. 

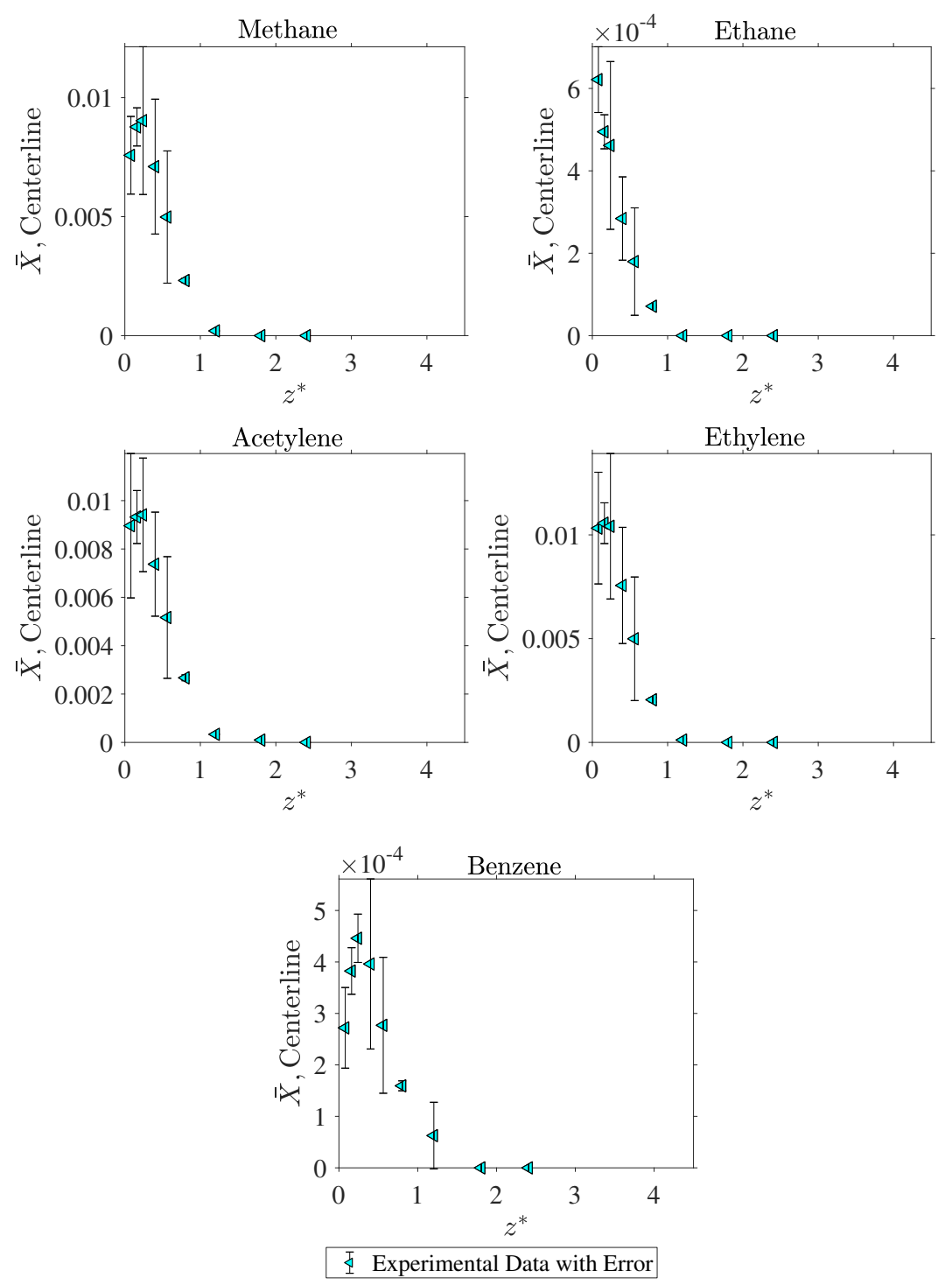

Fig. H.9. Plot of volume fractions of minor and trace species identified in the propane $(21 \mathrm{~kW})$ pool fire as a function of $z^{*}$ along the pool centerline. The error presented here is a combined uncertainty, further described in Section E. 


\section{H.6 Propane (34 kW)}
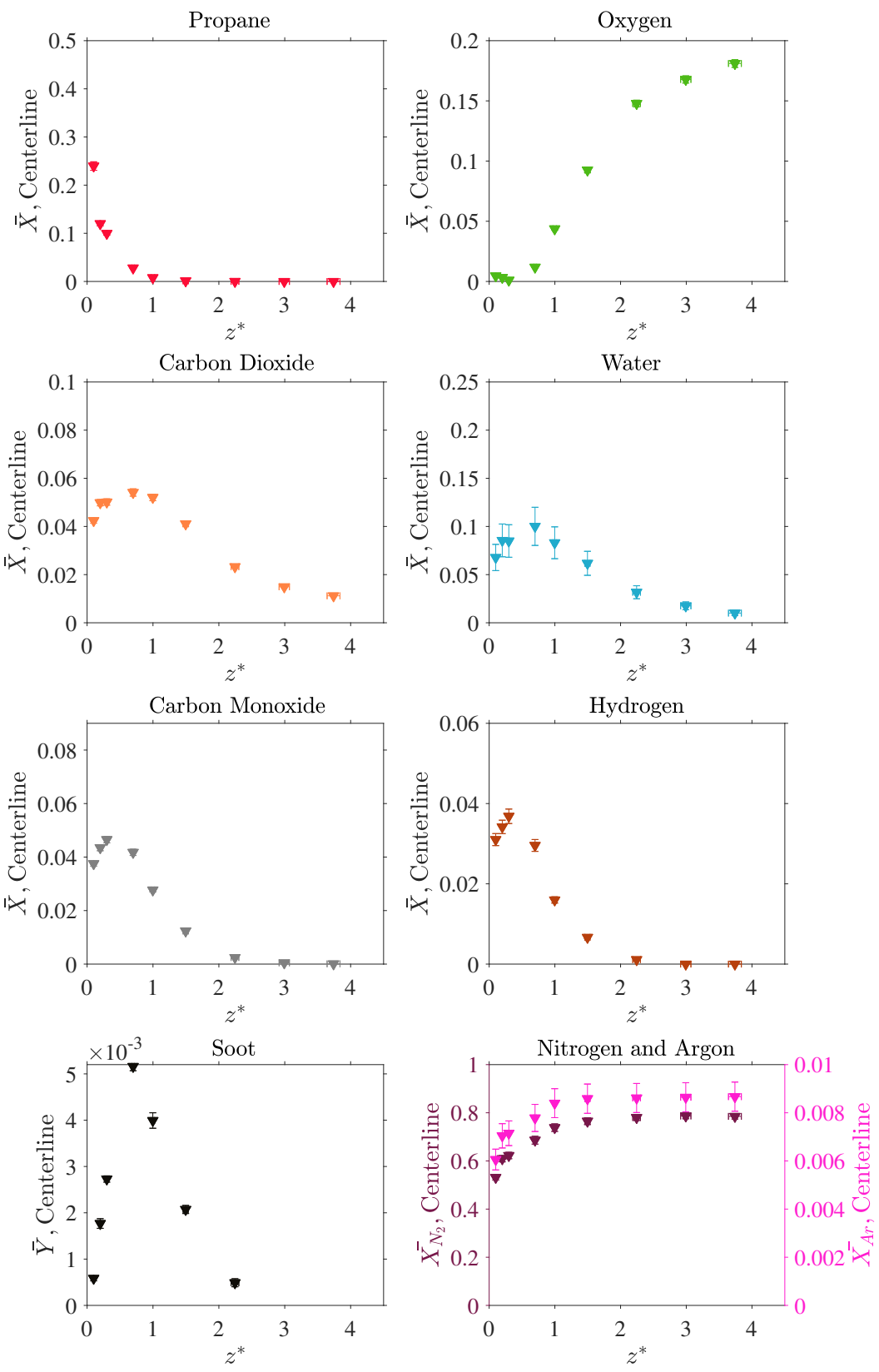

I Experimental Data with Error

Fig. H.10. Plot of volume fractions of all species identified in the propane $(34 \mathrm{~kW})$ pool fire as a function of $z^{*}$ along the pool centerline. The error is a combined uncertainty, further described in Section E. 

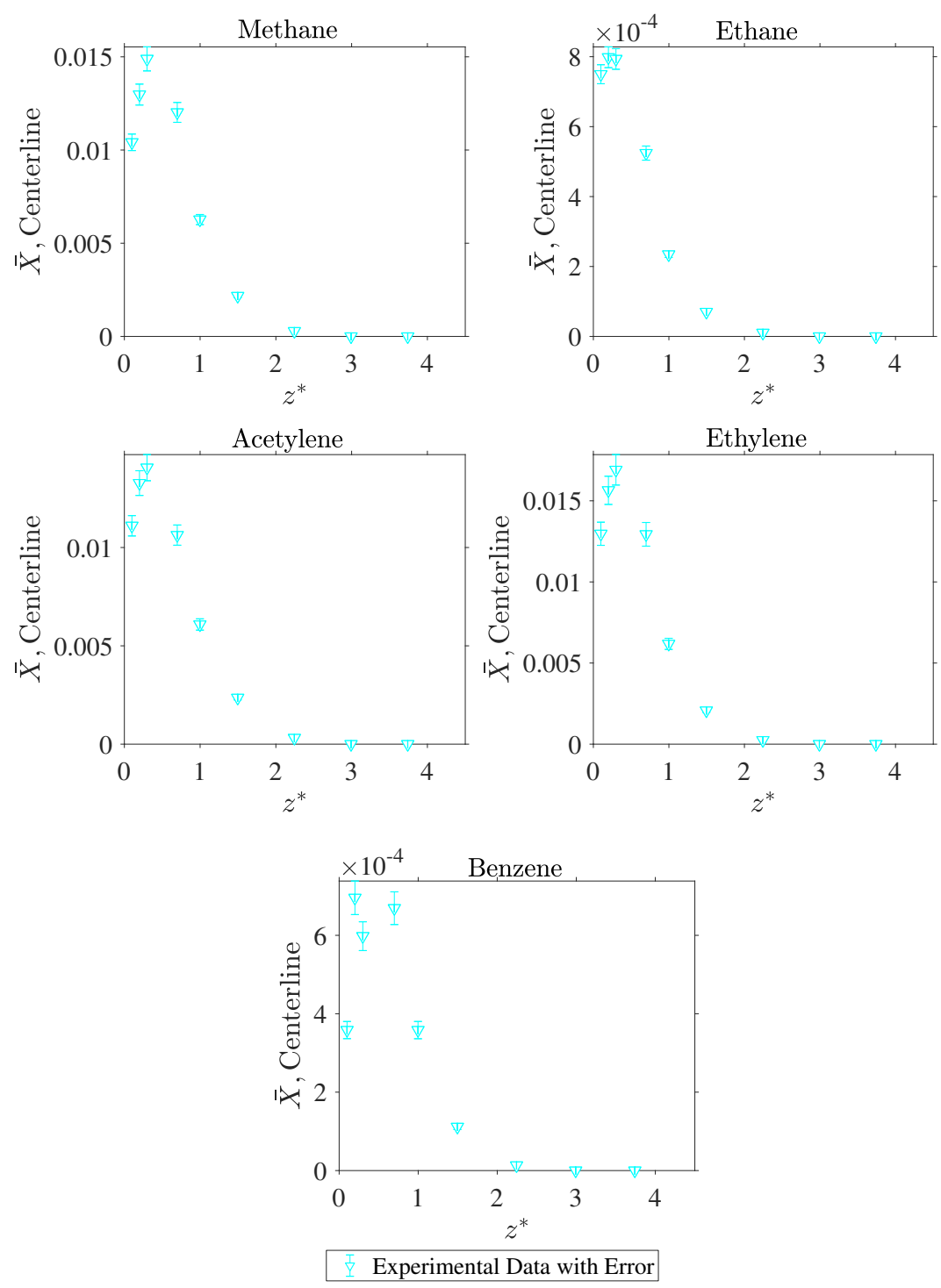

Fig. H.11. Plot of volume fractions of minor and trace species identified in the propane $(34 \mathrm{~kW})$ pool fire as a function of $z^{*}$ along the pool centerline. The error presented here is a combined uncertainty, further described in Section E. 


\section{Figures of Averaged Mass Fractions}

\section{I.1 Methanol}
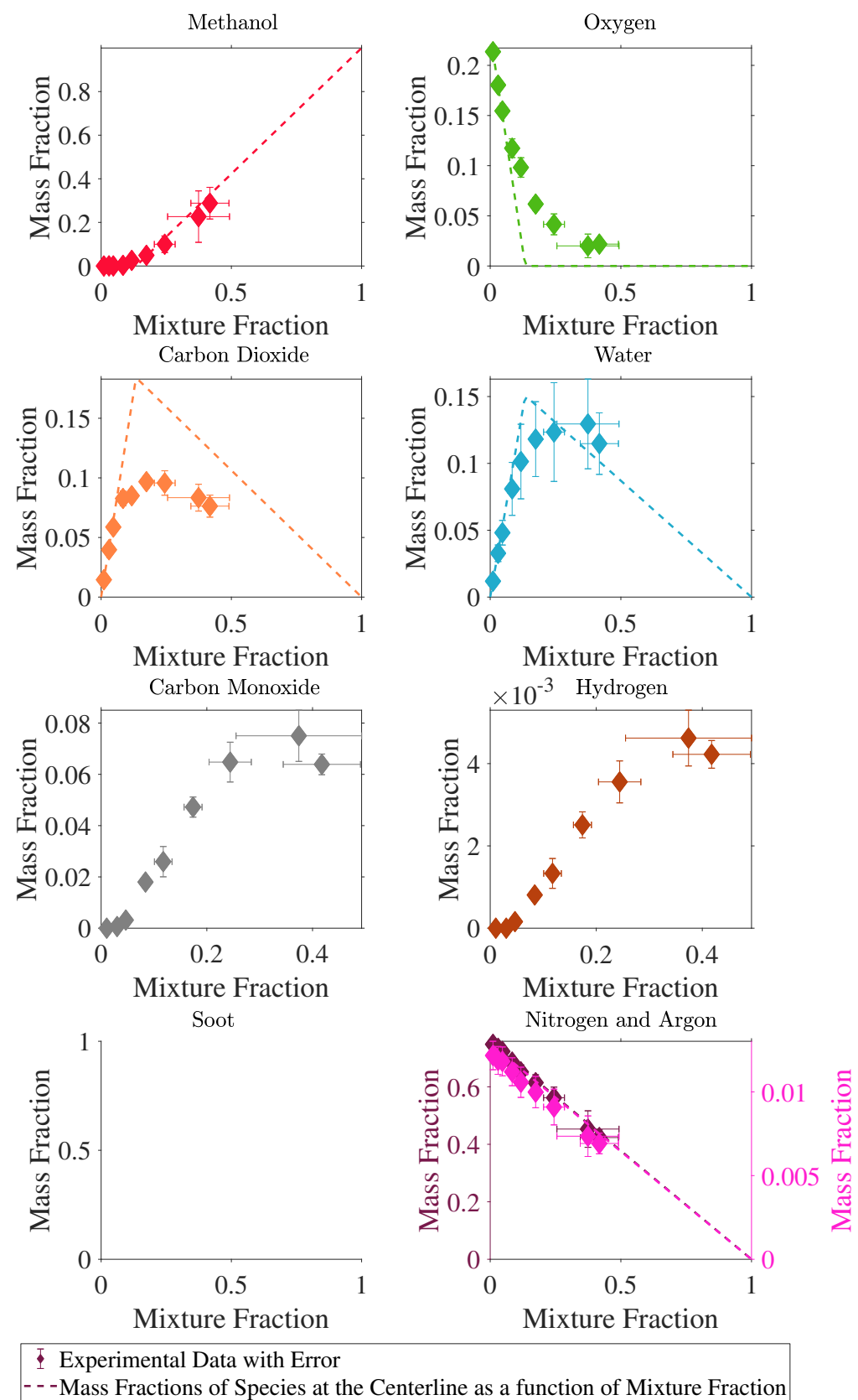

Fig. I.1. Plot of mass fractions, with uncertainty, of major species identified in the methanol pool fire centerline as function of mixture fraction. The uncertainty is a combined uncertainty, discussed in further detail in Sections $\mathrm{J}$ and $\mathrm{E}$ for the mass fraction and mixture fractions, respectively. 


\section{I.2 Ethanol}
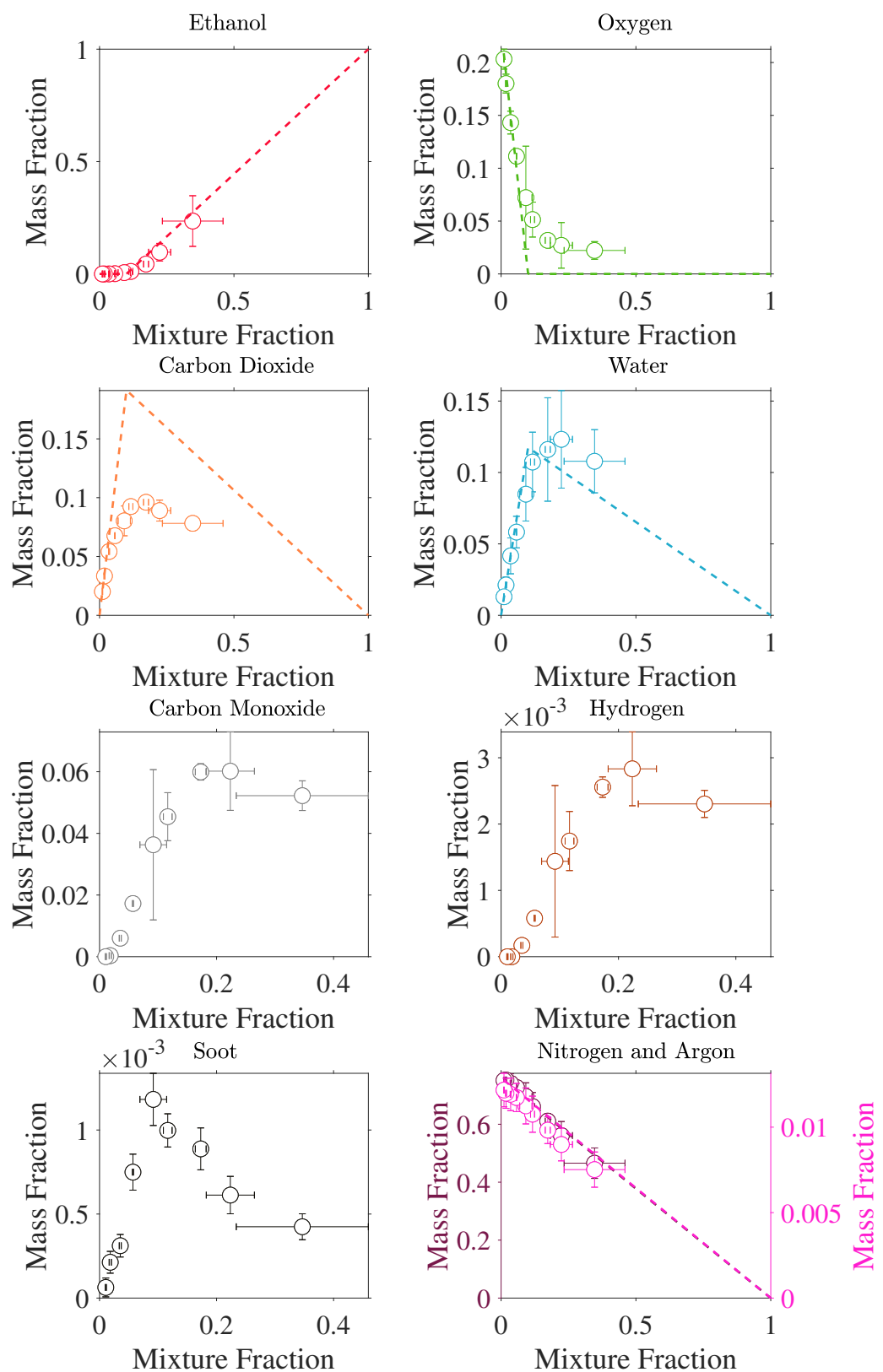

\section{Experimental Data with Error - - -Mass Fractions of Species at the Centerline as a function of Mixture Fraction}

Fig. I.2. Plot of mass fractions, with uncertainty, of major species identified in the ethanol pool fire centerline as function of mixture fraction. The uncertainty is a combined uncertainty, discussed in further detail in Sections $\mathrm{J}$ and $\mathrm{E}$ for the mass fraction and mixture fractions, respectively. 


\section{I.3 Acetone}
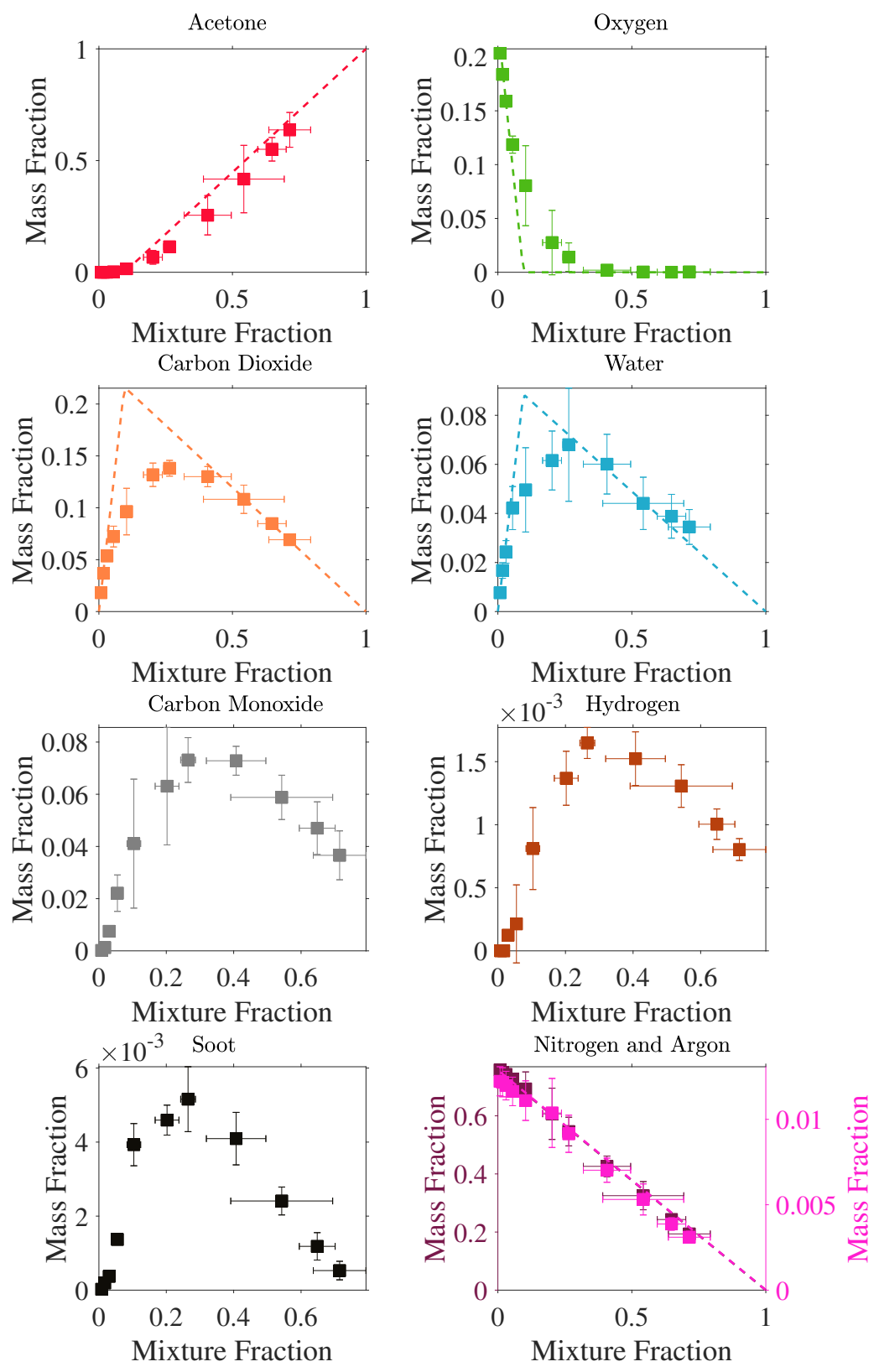

\footnotetext{
I Experimental Data with Error - - -Mass Fractions of Species at the Centerline as a function of Mixture Fraction
}

Fig. I.3. Plot of mass fractions, with uncertainty, of major species identified in the acetone pool fire centerline as function of mixture fraction. The uncertainty is a combined uncertainty, discussed in further detail in Sections $\mathrm{J}$ and $\mathrm{E}$ for the mass fraction and mixture fractions, respectively. 


\section{I.4 Methane}
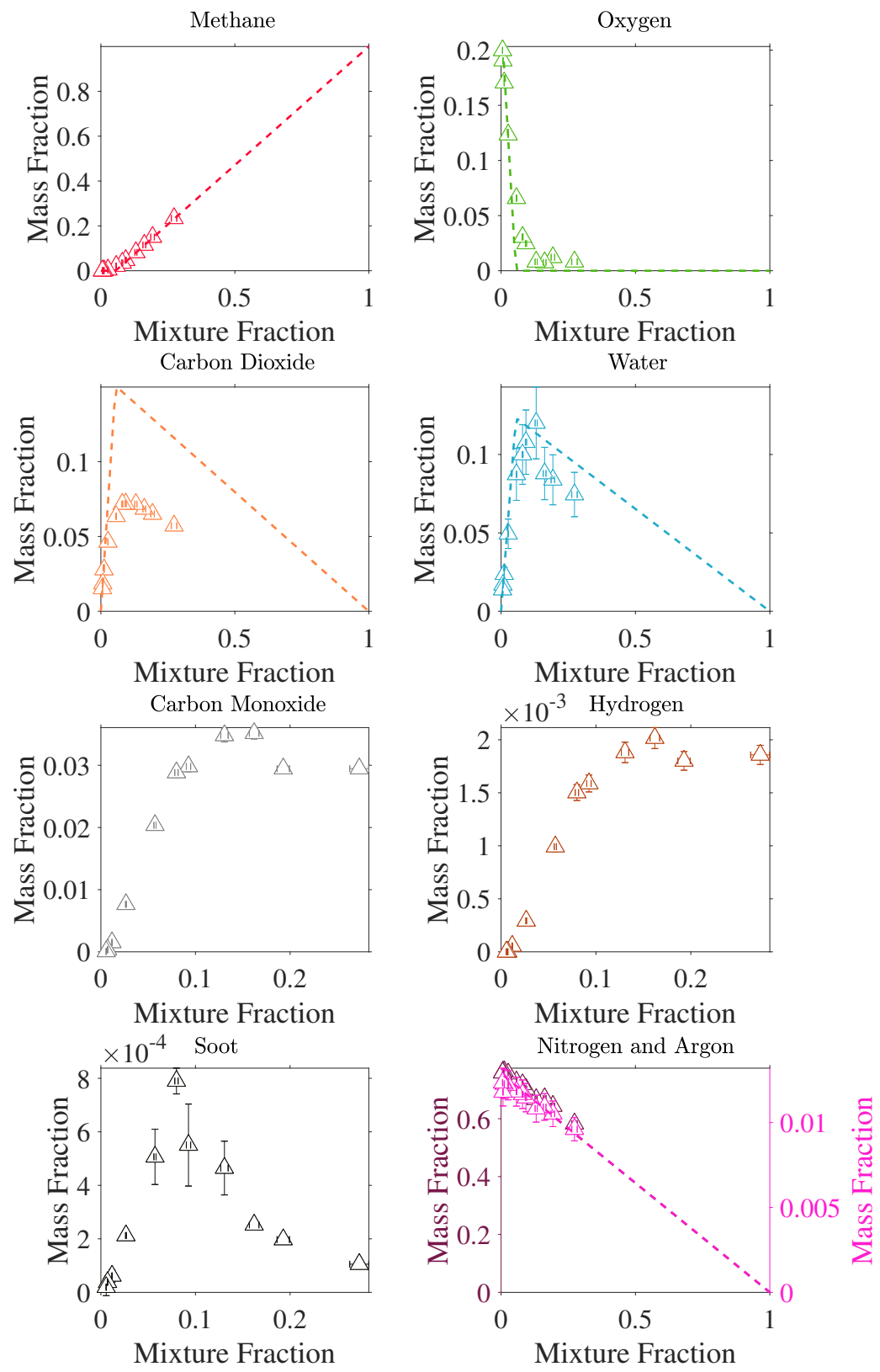

\footnotetext{
§ Experimental Data with Error - - -Mass Fractions of Species at the Centerline as a function of Mixture Fraction
}

Fig. I.4. Plot of mass fractions, with uncertainty, of major species identified in the methane pool fire centerline as function of mixture fraction. The uncertainty is a combined uncertainty, discussed in further detail in Sections $\mathrm{J}$ and $\mathrm{E}$ for the mass fraction and mixture fractions, respectively. 


\section{I.5 Propane $(21 \mathrm{~kW})$}
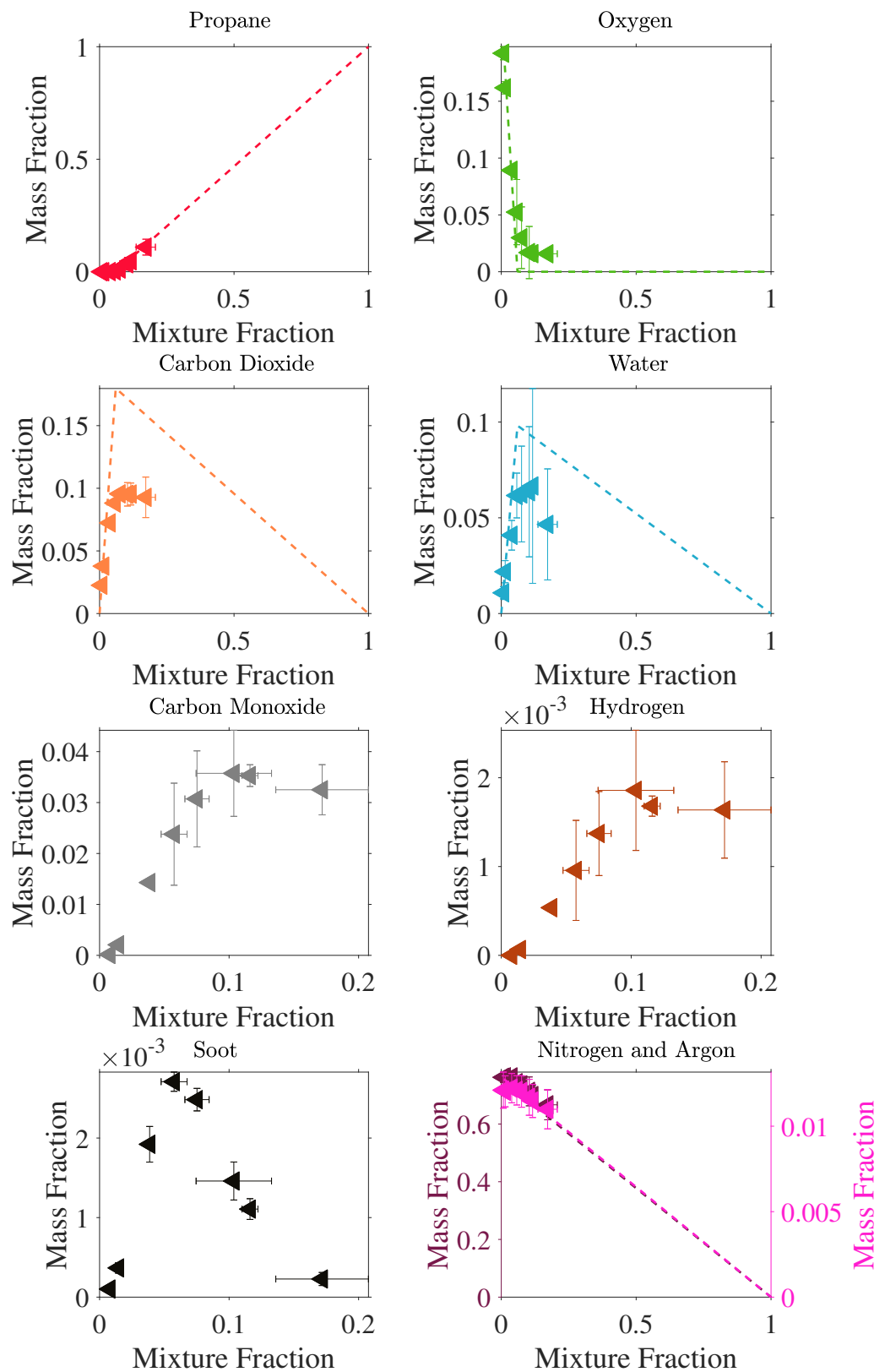

\footnotetext{
I Experimental Data with Error - - -Mass Fractions of Species at the Centerline as a function of Mixture Fraction
}

Fig. I.5. Plot of mass fractions, with uncertainty, of major species identified in the propane $(21 \mathrm{~kW})$ pool fire centerline as function of mixture fraction. The uncertainty is a combined uncertainty, discussed in further detail in Sections $\mathrm{J}$ and $\mathrm{E}$ for the mass fraction and mixture fractions, respectively. 


\section{I.6 Propane $(34 \mathrm{~kW})$}
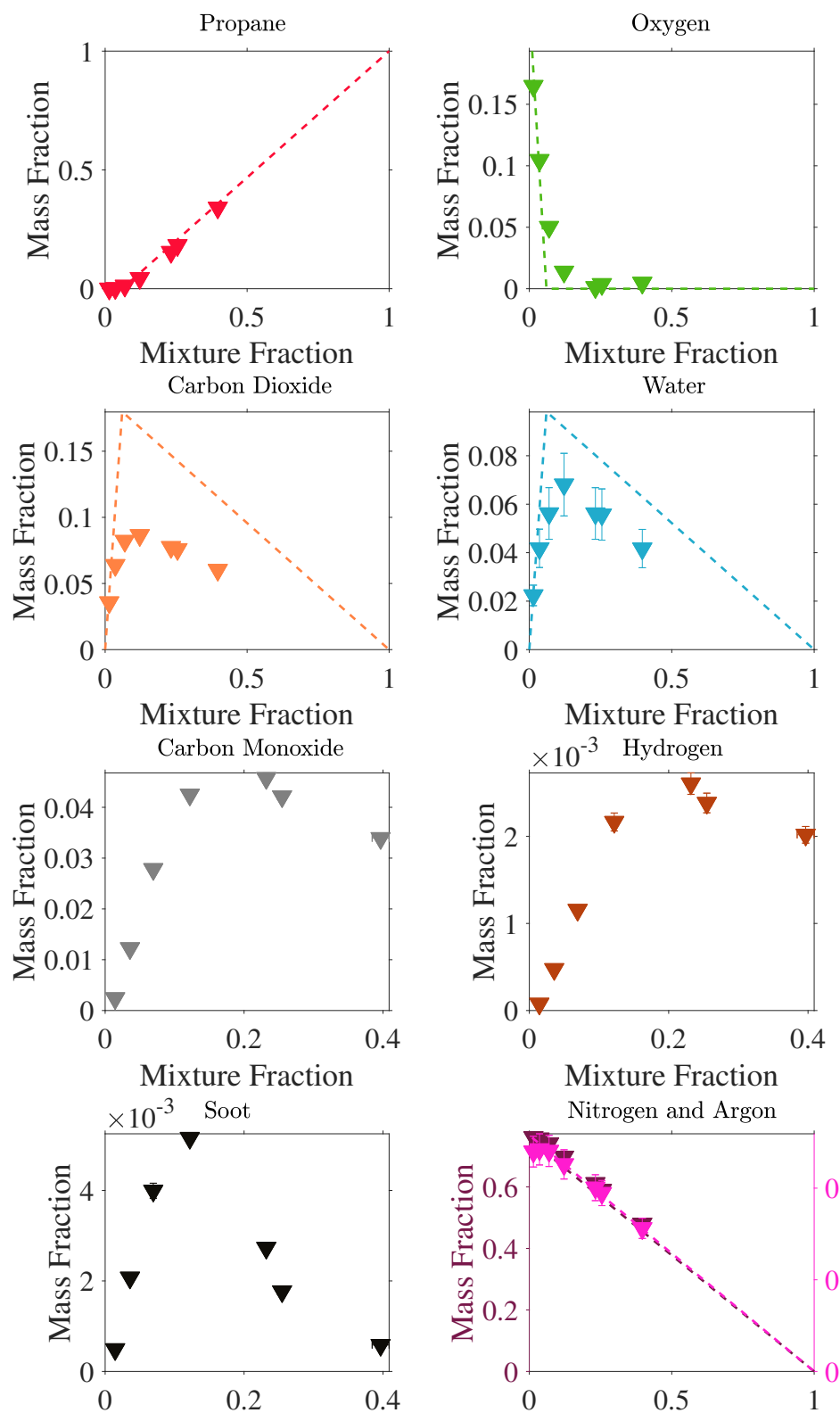

Mixture Fraction

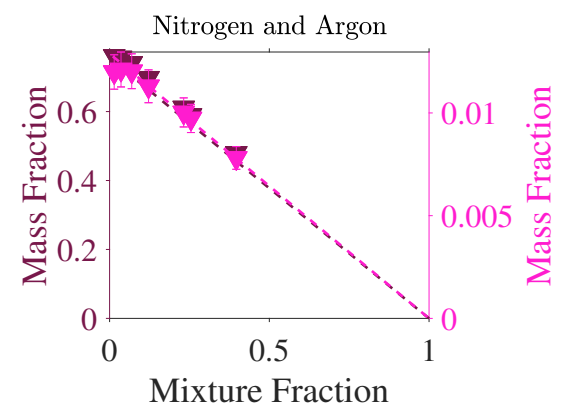

\footnotetext{
I Experimental Data with Error - - -Mass Fractions of Species at the Centerline as a function of Mixture Fraction
}

Fig. I.6. Plot of mass fractions, with uncertainty, of major species identified in the propane $(34 \mathrm{~kW})$ pool fire centerline as function of mixture fraction. The uncertainty is a combined uncertainty, discussed in further detail in Sections $\mathrm{J}$ and $\mathrm{E}$ for the mass fraction and mixture fractions, respectively. 


\section{J. Uncertainty Analysis of the Verification Scheme}

\section{J.1 Ratio of moles identified to moles injected}

The uncertainty of the ratio of the total number of moles identified by the TCD and MS, $n_{\text {tot }}$, to the number of moles injected into the GC/MS, $n_{\text {inj }}$, is calculated using the law of propagation of uncertainty and shown in the equation below:

$$
u_{n_{\mathrm{tot}} / n_{\mathrm{inj}}}=\sqrt{\left(\frac{u_{n_{\mathrm{tot}}}}{n_{\mathrm{inj}}}\right)^{2}+\left(\frac{-n_{\mathrm{tot}} u_{n_{\mathrm{inj}}}}{n_{\mathrm{inj}}^{2}}\right)^{2}}
$$

The uncertainty of the total number of moles identified, $n_{\mathrm{tot}}$, is described in Section E.1.2. The uncertainty of the number of moles injected into the GC/MS, $n_{\text {inj }}$, is described in Section F.1.

\section{J.2 Carbon to Hydrogen Ratio}

The carbon to hydrogen ratio is calculated using Eq. 17 using the mole fraction of any quantified gas species that contained either carbon or hydrogen.

$$
u_{\mathrm{C} / \mathrm{H}}=\sqrt{\sum\left(\frac{\partial(\mathrm{C} / \mathrm{H})}{\partial \bar{X}_{i}} u_{\bar{X}_{i}}\right)^{2}+\sum\left(\frac{\partial(C / H)}{\partial X_{i}} u_{\bar{X}_{i}}\right)^{2}}
$$

The uncertainty of the volume fractions of carbon and hydrogen containing species is described in Appendix E.2.

\section{J.3 Inert Ratio}

The inert ratio is calculated from the ratio the Nitrogen and Argon volume fractions, $\bar{X}_{N_{2}}$ and $\bar{X}_{A r}$, identified by the TCD and TIC chromatograms. The uncertainty of inert ratio is determined from the law of propagation of uncertainty:

$$
u_{\bar{X}_{A r} / \bar{X}_{N_{2}}}=\sqrt{\left(\frac{u_{\bar{X}_{A r}}}{\bar{X}_{N_{2}}}\right)^{2}+\left(\frac{-\bar{X}_{A r} u_{\bar{X}_{N_{2}}}}{\bar{X}_{N_{2}}^{2}}\right)^{2}}
$$

The uncertainty of the volume fractions is described in Appendix E.1.

\section{J.4 Uncertainty Analysis of the Mixture Fraction}

The mixture fraction, $Z$, is determined from Eq. (18). The uncertainty of the mixture fraction is a function of the uncertainties in the carbon carrying species:

$$
u_{Z}=\sqrt{u_{\bar{Y}_{\mathrm{F}}}^{2}+\left(\frac{W_{\mathrm{F}}}{\mathrm{x}}\right)^{2} \sum_{i \neq \mathrm{F}}\left(\frac{u_{\bar{Y}_{i}}}{\mathrm{~W}_{i}}\right)^{2}}
$$


The uncertainty of the mass fractions of carbon containing species is discussed in Appendix E.2. 


\section{K. Previous Measurements in $30 \mathrm{~cm}$ Pool Fires}

Many experiments have been conducted on pool fires such as those considered in this report [2-4, 7, 8, 10, 17, 24-28]. Table K.1 lists previously reported local and global measurements characterizing the structure of $30 \mathrm{~cm}$ diameter methanol, ethanol and acetone pool fires steadily burning under well-ventilated and quiescent conditions. These measurements complement each other and help build a more complete picture of the energetics, structure and dynamics of medium-scale pool fires burning oxygenated fuels. The accumulated information provides a basis for understanding details about these fires that makes them particularly suitable candidates for fire model evaluation. The three fires considered here are particularly useful as a testbed for radiation sub-models since blackbody radiation from soot is dependent on the fuel type. The time-averaged mean and RMS data from several of the studies is available through the Measurement and Computational Fire Phenomena (MaCFP) GitHub website [29].

Table K.1. List of previously measured parameters obtained from a well-ventilated, round, steady, $30 \mathrm{~cm}$ diameter pool fires burning in a quiescent environment.

\begin{tabular}{|c|c|c|c|c|}
\hline & \multirow{2}{*}{ Parameter } & \multicolumn{3}{|c|}{ References } \\
\hline & & Methanol & Ethanol & Acetone \\
\hline \multirow{5}{*}{$\begin{array}{l}\bar{\sigma} \\
\text { ㅇ } \\
0\end{array}$} & Mass Loss Rate & [4] & & [26] \\
\hline & Heat Release Rate & [8] & & [27] \\
\hline & Mean Flame Height & {$[4,7,24,25]$} & {$[4,7,25]$} & {$[7,25,26]$} \\
\hline & Pulsation Frequency & {$[2-4,7,8,24,28]$} & {$[7,25,28]$} & {$[7,25,27,28]$} \\
\hline & Radiative Fraction & [7] & [7] & [7] \\
\hline \multirow{7}{*}{$\begin{array}{l}\bar{U} \\
0 \\
0\end{array}$} & Radiative flux distribution on fuel surface & [3] & & \\
\hline & Total Heat Flux Distributuion on Fuel Surface & [7] & [7] & [7] \\
\hline & Radiative flux to surroundings & {$[4,7,24]$} & & \\
\hline & Transient fuel temperature & [3] & & \\
\hline & Gas species volume fraction & [2] & & \\
\hline & Local Gas Phase Temperature & {$[4,8]$} & & \\
\hline & Local Gas Phase Velocity & [8] & & \\
\hline
\end{tabular}

There are subtle differences in the experimental conditions as well as reporting assumptions and approximations that may account for some of the differences among the reported results. Some of the relevant issues are highlighted in Table K.2 below for the methanol, ethanol and acetone pool fires, respectively. The tables include information on the steadystate mass burning flux, $m^{\prime \prime}$, lip height, mean flame height.

The radiative fraction to the surroundings including the fuel pool, $\chi_{\mathrm{rad}}$, and excluding the fuel pool, $\chi_{\mathrm{r}}$, during steady burning is reported for each study. $\chi_{\text {rad }}$ is defined as the total radiative heat transfer to the surroundings and onto the fuel surface such that:

$$
\chi_{\mathrm{rad}}=\chi_{\mathrm{r}}+\chi_{\mathrm{sr}}
$$

where $\chi_{\mathrm{r}}$ is the integrated radiative flux emitted by the fire in all directions except to the fuel surface, normalized by the fire heat release rate. The term $\chi_{\text {sr }}$ represents the integrated 
radiative flux emitted by the fire towards the fuel surface, normalized by the fire heat release rate. The values of the fractional enthalpy terms $\left(\chi_{\mathrm{r}}, \chi_{\mathrm{sr}}\right)$ vary from fire to fire and are dependent on fuel type, burner diameter, and fire size. Ref. [7] describes the development of these terms in more detail.

There are several general considerations regarding the previous work:

- Ref. [8] reports using a round, stainless-steel, $30.5 \mathrm{~cm}$ diameter burner, which differs from the $30.1 \mathrm{~cm}$ (inner) diameter burner used in Refs. [2-4, 7, 10, 24, 25]. Due to this disparity ( $3 \%$ in area), Table K.1 refers to the mass burning flux (rather than the mass burning rate). The same circular, water-cooled, stainless-steel pan was used for experiments $[2-4,7,10,24,25]$. The burner had an inner diameter of $30.1 \mathrm{~cm}$, a depth of $15 \mathrm{~cm}$, and a wall thickness of $1.6 \mathrm{~mm}$. The burner was fitted with legs such that the burner rim was positioned $30 \mathrm{c} \mathrm{m}$ above the ground. The bottom of the burner was maintained at a constant temperature by flowing tap water (nominally $20{ }^{\circ} \mathrm{C}$ ) through a $3 \mathrm{~cm}$ section on the bottom of the fuel pan. The exhaust flow in [7] was $0.50 \mathrm{~kg} / \mathrm{s}$; this value can be assumed to be approximately the same for Refs. [2, 3, 10, 24, 25].

- The lip height reported in Ref. [8] and several of the other studies was $10 \mathrm{~mm}[2,10]$, except Refs. [2-4, 24, 25] where it was reported as $4 \mathrm{~mm}$ or $5 \mathrm{~mm}$ (see Table K.1).

- Weckman and Strong's results [8] and Ref. [2] are presented as a function of distance from the fuel surface, whereas all the other studies use the top of the burner rim as the point of reference.

- A water-cooling section was not reported on the bottom of the burner used in Ref. [8]; the burner in Refs. [2-4, 7, 10, 24, 25] had a water-cooling section on the bottom of the burner.

- Refs. [7, 25] reported radiative fraction emitted to the surroundings and did not include flux incident on the fuel surface; Ref. [7] reported radiative fraction to the surroundings plus flux incident on the fuel surface; Ref. [24] assumed flux incident on the pool was uniform across the pool and equal to its value just outside the pool burner.

- The amount of $\mathrm{CO}$ in the methanol, ethanol and acetone exhaust stream was below detection limits [7], so the combustion efficiency can be assumed to be about 1 .

- In Ref. [24], the average heat flux to the pool was assumed to be equal to the radiative flux measured just outside the burner, which is smaller than that expected [7]. Here, $\chi_{\mathrm{rad}}$ is recalculated using $\chi_{\mathrm{r}}=0.065$ from [7].

- In Ref. [24], $\chi_{\text {rad }}$ was based on a heat of combustion of $22.317 \mathrm{~kJ} / \mathrm{g}$. Here, $\chi_{\text {rad }}$ is recalculated using the "net" heat of combustion $(19.94 \mathrm{~kJ} / \mathrm{g})$ in which gaseous water is assumed to be the product of combustion [9]. 
- The heat of combustion values provided in Table 1 were used to determine the heat release rates reported in Table K.2.

Table K.2. Reported mass burning flux, $m^{\prime \prime}$, heat release rate, $\dot{Q}$, radiative fractions, excluding, $\chi_{\mathrm{r}}$, and including, $\chi_{\mathrm{rad}}$ the fuel pool, and the lip height of steady burning pool fires

\begin{tabular}{|c|c|c|c|c|c|c|c|}
\hline & $\dot{m}^{\prime \prime}\left(\mathrm{g} / \mathrm{m}^{2} \mathrm{~s}\right)$ & $\dot{Q}(\mathrm{~kW})$ & $\chi_{\mathrm{r}}$ & $\chi_{\text {rad }}$ & $\begin{array}{l}\text { Lip } \\
\text { Heigh }\end{array}$ & Ref. & Comments \\
\hline \multirow{11}{*}{ 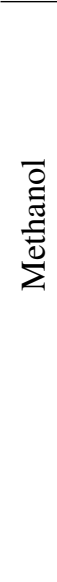 } & 15.1 & 21.4 & & & 10 & [8] & $\begin{array}{l}\text { Volumetric Burning } \\
\text { Rate of } 1.35 \mathrm{~cm}^{3} / \mathrm{s}\end{array}$ \\
\hline & $13.5 \pm 1.2$ & 19.2 & 0.19 & 0.24 & 10 & [7] & \multirow{10}{*}{$\begin{array}{l}\text { Fuel Mass Burning } \\
\text { Rate of } 0.92 \mathrm{~g} / \mathrm{s} \\
\text { Fuel Mass Burning } \\
\text { Rate of } 0.9 \mathrm{~g} / \mathrm{s}\end{array}$} \\
\hline & 12.9 & 18.3 & 0.22 & & 5 & [3] & \\
\hline & 12.6 & 18.0 & & 0.20 & 5 & [24] & \\
\hline & & 20 & 0.18 & 0.22 & 4 & [4] & \\
\hline & & & & & 5 & [2] & \\
\hline & & & & & 10 & [10] & \\
\hline & $12.8 \pm 1.3$ & 18.2 & 0.22 & & 5 & [25] & \\
\hline & $12.4 \pm 0.5$ & 17.6 & & & 107 & This study & \\
\hline & $12.4 \pm 0.5$ & 17.6 & & & 107 & This study & \\
\hline & $13.2 \pm 0.4$ & $18.7 \pm 0.6$ & $0.20 \pm 0.02$ & $0.22 \pm 0.2$ & & Average & \\
\hline \multirow{4}{*}{$\begin{array}{l}\overline{0} \\
\text { 胥 } \\
\text { III }\end{array}$} & $16.2 \pm 1.8$ & 31.0 & 0.21 & & 10 & [7] & \\
\hline & $14.4 \pm 1.4$ & 27.3 & 0.16 & & 5 & [25] & \\
\hline & $13.9 \pm 2.1$ & 26.3 & & 0.26 & 107 & This study & \\
\hline & $14.8 \pm 1.2$ & $28.2 \pm 2.5$ & $0.19 \pm 0.3$ & 0.26 & & Average & \\
\hline \multirow{5}{*}{$\begin{array}{l}\frac{0}{\tilde{z}} \\
\frac{0}{0} \\
\frac{\tilde{U}}{2}\end{array}$} & 25.9 & 54.1 & 0.26 & \multirow{4}{*}{0.31} & 10 & [8] & \multirow[t]{5}{*}{$\dot{Q}^{\prime \prime}$ of $740 \mathrm{~kW} / \mathrm{m}^{2}$} \\
\hline & $18.7 \pm 2.1$ & 38.1 & 0.27 & & 10 & [7] & \\
\hline & $18.7 \pm 1.9$ & 37.7 & 0.28 & & 5 & [25] & \\
\hline & $17.6 \pm 2.6$ & 35.5 & & & $10 \mathrm{~T}$ & This Study & \\
\hline & $18.3^{* *} \pm 0.6$ & $37.1 \pm 1.4$ & $0.27 \pm 0.1$ & 0.31 & & Average & \\
\hline
\end{tabular}

** The mass burning flux of acetone reported in [8] is an outlier and not included in the average.

Uncertainty of averages is defined as the standard deviation with a coverage factor of 2. 